\title{
Extensões de distribuições com aplicação à analise de sobrevivência
}

\author{
Yolanda Magaly Gómez Olmos \\ TESE APRESENTADA \\ $\mathrm{AO}$ \\ Instituto de Matemática e Estatística \\ DA \\ Universidade De SÃo Paulo \\ PARA \\ OBTENÇÃO DO TÍTULO \\ DE \\ DOUTOR EM CIÊNCIAS \\ Programa: Estatística \\ Orientador: Prof. Dr. Heleno Bolfarine
}

Durante o desenvolvimento deste trabalho a autora recebeu auxílio financeiro de Conicyt

(Comisión Nacional de Investigación, Ciencia y Tecnología - Chile)

São Paulo, Fevereiro de 2017 



\title{
Extensões de distribuições com aplicação \\ à analise de sobrevivência
}

\author{
Esta tese contém as correções e alterações \\ sugeridas pela Comissão Julgadora durante a defesa \\ realizada por Yolanda Magaly Gómez Olmos em 09/02/2017. \\ O original encontra-se disponível no Instituto de \\ Matemática e Estatística da Universidade de São Paulo.
}

Comissão Julgadora:

- Prof. Dr. Heleno Bolfarine (orientador) - IME-USP

- Prof. Dr. Reinaldo Boris Arellano Valle - PUCCH-Externo

- Prof. Dr. Mário de Castro Andrade Filho - ICMC-USP

- Prof. Dr. Josemar Rodrigues - ICMC-USP

- Prof. Dr. Alexandre Galvão Patriota - IME-SUP 
$E$ disse ao homem:

Eis que o temor do Senhor é a sabedoria, e apartar-se do mal é a inteligência.

Jó 28:28

$Y$ dijo al hombre: He aqui que el temor del Señor es la sabiduría, $Y$ el apartarse del mal, la inteligencia. Job 28:28 


\section{Agradecimentos}

A meus pais (Neveka e Héctor), por seu apoio incondicional desde o primeiro momento que decidi fazer o doutorado, longe da minha cidade, de meu país. Suas constantes mensagens e ligações, tornaram suportável minha estadia. A meus irmãos (Belén e Josué) por alegrar minha vida, eu amo muito vocês.

A meu marido Diego, por todo seu apoio e paciência, muito especialmente na etapa final. A minha princesinha Larissa, por chegar a mudar toda minha vida, por estar no momento da minha qualificação na barriga dando-me apoio, e agora já na etapa final. Eu amo muito vocês meus amores.

Ao professor Heleno, por ter aceitado orientar-me e pelas valiosas sugestões ao longo deste trabalho.

Aos professores do IME, que foram fundamentais no meu crecimento académico, tais como os professores Carlos Bragança Pereira, Denise Aparecida Botter, Lucia Pereira Barroso, Silvia Ferrari, Marcia D'Elia Branco, Gisela Tunes, Gilberto Paula, Antonio Carlos Predroso.

Ao professor Barry Arnold, por sua participação como co-autor no desevolvimento do capítulo 4 desta tese.

Á banca julgadora, por suas correções e sugestões. Certamente ajudaram a melhorar a apresentação deste trabalho.

Aos amigos que conheci no IME, Elizabeth González, Fran Lima, Alejandra Tapia, Manuel González, especialmente a minha amiga Natalia Milla, por todo seu apoio no começo do dourorado, por ficar a meu lado cada vez que sentia saudade, pelas palavras de encorajamento, por isso e muito mais, muito obrigada!

Finalmente, agradeço o apoio financeiro por parte da CAPES (Coordenação de aperfeiçoamento de pessoal de nivel superior) que foi fundamental para minha chegada ao Brasil e para o financiamento dos vários congressos que assisti. Também agradeço a CONICYT (Comisión Nacional de Investigación, Ciencia y Tecnología) através de seu programa BECAS CHILE para Doutorado no estrangeiro, por ter financiado a continuação deste Doutorado. 


\section{Resumo}

Nesta tese serão estudadas diferentes generalizações de algumas distribuições bem conhecidas na literatura para os tempos de vida, tais como exponencial, Lindley, Rayleigh e exponencial segmentada, entre outras, e compará-las com outras extensões com suporte positivo. A finalidade dessas generalizações é flexibilizar a função de risco de modo que possam assumir formas mais flexíveis. Além disso, pretende-se estudar propriedades importantes dos modelos propostos, tais como os momentos, coeficientes de curtose e assimetria e função quantílica, entre outras. A estimação dos parâmetros é abordada através dos métodos de máxima verossimilhança, via algoritmo EM (quando for possível) ou também, do método dos momentos. O comportamento desses estimadores foi avaliado em estudos de simulação. Foram ajustados a conjuntos de dados reais, usando uma abordagem clássica, e compará-los com outras extensões na literatura. Finalmente, um dos modelos propostos é considerado no contexto de fração de cura.

Palavras-chaves: Análise de sobrevivência, Fração de cura, Máxima verossimilhança. 


\begin{abstract}
The main focus of this thesis is the study of generalizations for some positive distributions widely known in the literature of lifetime analysis, such as the exponential, Lindley, Rayleigh and segmented exponential. Comparisons of the proposed extensions and alternative extensions in the literature such as the generalized exponential distribution, are reported. Moreover, of interest is also the study of some properties of the proposed distributions such as moments, kurtosis and asymmetry coefficients, quantile functions and the risk function. Parameter estimation is approached via maximum likelihood (using the EM-algorithm when available) and the method of moments as initial parameter estimators. Results of simulation studies are reported comparing the performance of these estimators with small and moderate sample sizes. Further comparisons are reported for real data applications, where the proposed models show satisfactory performance.
\end{abstract}

Keywords: Survival analysis, Cure rate, Maximum Likelihood. 


\section{Sumário}

Lista de Abreviaturas $\quad$ xi

Lista de Figuras $\quad$ xiii

Lista de Tabelas $\quad$ xv

1 Motivação $\quad 1$

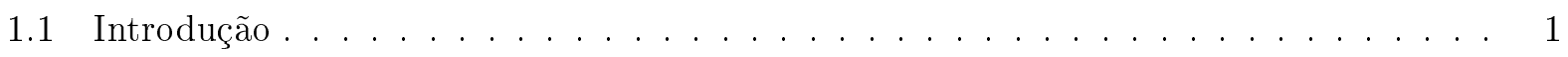

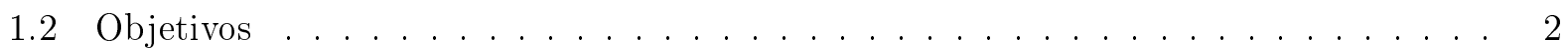

1.3 Organização da Tese . . . . . . . . . . . . . . . . . . . 2

2 Preliminares $\quad 5$

2.1 Conceitos básicos em análise de sobrevivência . . . . . . . . . . . . . . 5

2.1 .1 Função de sobrevivência . . . . . . . . . . . . . . . . . . 5

2.1.2 Função de risco ou função de taxa de falha . . . . . . . . . . . . . . . 5

2.2 Principais distribuições com suporte positivo $\ldots \ldots \ldots \ldots \ldots \ldots$

2.3 Algumas extensões de distribuições conhecidas . . . . . . . . . . . . . . . . . 15

2.3.1 Half-normal generalizada [Cooray e Ananda [2008]] . . . . . . . . . . . . . 18

2.3.2 Exponencial generalizada [Gupta e Kundu [1999]] . . . . . . . . . . . . . . . . 18

2.3.3 Uma extensão do modelo exponencial [Nadarajah e Haghighi [2011]] . . . . 19

2.4 Principais testes de bondade de ajuste . . . . . . . . . . . . . . . . . . . 19

2.4 .1 Teste de Kolmogorov-Smirnov . . . . . . . . . . . . . . . . . 20

2.4 .2 Teste da razão de verossimilhanças (TRV) . . . . . . . . . . . . . . . . . 20

2.4 .3 Testes Anderson-Darling e Cramér-von Mises . . . . . . . . . . . . . . . 21

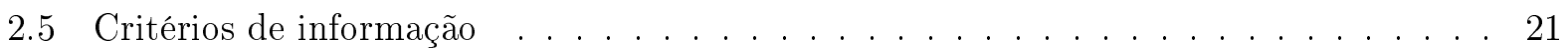

2.5.1 Critério de informação de Akaike . . . . . . . . . . . . . . . 22

2.5.2 Critério de informação de Akaike consistente . . . . . . . . . . . . . . . 22

2.5.3 Critério de informação Bayesiano . . . . . . . . . . . . . . . . 22

3 Distribuição exponencial estendida $\quad 23$

3.1 Motivação . . . . . . . . . . . . . . . . . . . . . . 23

3.2 Funções de densidade e propriedades . . . . . . . . . . . . . . . . . . 23

3.2 .1 Principais propriedades . . . . . . . . . . . . . . . 24

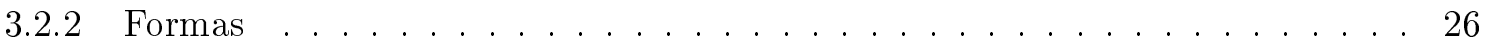

3.3 Função quantílica . . . . . . . . . . . . . . . . . . . . . . . 26

3.4 Entropia de Shannon . . . . . . . . . . . . . . . . . . . . 28

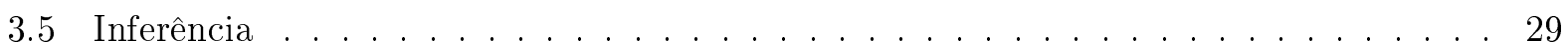


3.5.1 Estimação pelo método dos momentos . . . . . . . . . . . . . . 29

3.5.2 Estimadores de máxima verossimilhança $(\mathrm{EMV}) \ldots \ldots$. . . . . . . . . 29

3.6 Ilustração . . . . . . . . . . . . . . . . . . . . . . . . . . 30

3.6.1 Filamentos de Kevlar 49/epoxy a um de nível de estresse de $74.8 \%$. . . . . . 30

3.7 Observações finais . . . . . . . . . . . . . . . . . . . . . 30

4 O modelo Rayleigh-Lindley 33

4.1 Motivação . . . . . . . . . . . . . . . . . . . . . . . . . 33

4.2 O modelo . . . . . . . . . . . . . . . . . . . . . . . 34

4.2 .1 Funções de densidade e sobrevivência . . . . . . . . . . . . . . . . . . . 34

4.3 Principais propriedades . . . . . . . . . . . . . . . . . 35

4.3.1 Momentos e medidas complementares . . . . . . . . . . . . . . . 35

4.3 .2 Vida média residual . . . . . . . . . . . . . . . . . . . . . 37

4.4 Estimação . . . . . . . . . . . . . . . . . . . . . . . 37

4.4 Método dos momentos . . . . . . . . . . . . . . . . . . . 37

4.4.2 Algoritmo EM para dados censurados . . . . . . . . . . . . . . . . 38

4.5 Estudo de simulação . . . . . . . . . . . . . . . . . . . . . . . . . 39

4.6 Ilustração . . . . . . . . . . . . . . . . . . . . . . . . . . . 43

4.6.1 Tempos de sobrevivência de cobaias . . . . . . . . . . . . . . . . 43

4.6.2 Tempos de falha de componentes . . . . . . . . . . . . . . . . . . . 43

4.7 Observações finais . . . . . . . . . . . . . . . . . . . . . 45

5 Distribuição exponencial segmentada potência $\quad 47$

5.1 Motivação . . . . . . . . . . . . . . . . . . . . . . . . . 47

5.2 Modelo exponencial segmentado potência . . . . . . . . . . . . . . . 48

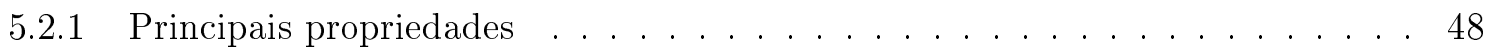

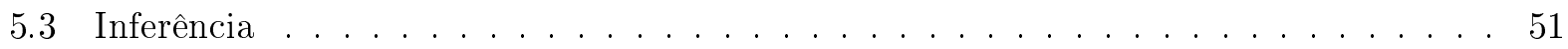

5.3 .1 A função de log-verossimilhança . . . . . . . . . . . . . . . . . 51

5.3 .2 A função escore . . . . . . . . . . . . . . . . . . . . . 51

5.3 .3 Matriz de informação observada . . . . . . . . . . . . . . . . . 52

5.3.4 Função de log-verossimilhança com presença de censura à direita . . . . . . . 52

5.4 Estudo de simulação . . . . . . . . . . . . . . . . . . . . . . . . 53

5.5 Aplicações . . . . . . . . . . . . . . . . . . . . . . 55

5.5.1 Filamentos de Kevlar 49 /epoxy a 90\% de nível de estresse (exemplo sem

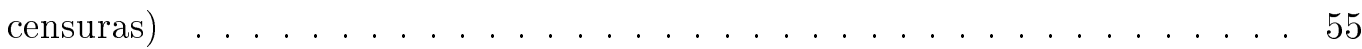

5.5.2 Estudo de radioterapia (dados censurados) . . . . . . . . . . . . . . 57

5.6 Observações finais . . . . . . . . . . . . . . . . . . . . 59

6 Distribuição half-normal potência $\quad 61$

6.1 Motivação . . . . . . . . . . . . . . . . . . . . . . . 61

6.2 Distribuição half-normal potência . . . . . . . . . . . . . . . . . 61

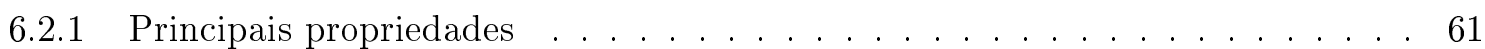

6.2 .2 Caracterizações da distribuição $\mathrm{PHN} \ldots \ldots \ldots$. . . . . . . . . . . . 62

6.2 .3 Momentos . . . . . . . . . . . . . . . . . . . . . . . . . . 64 
6.2 .4 Entropia de Shannon . . . . . . . . . . . . . . . . . . . 65

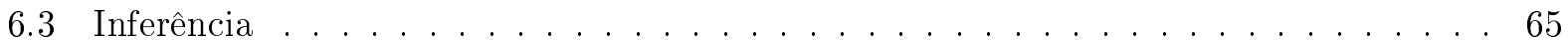

6.3.1 Estimadores de momentos . . . . . . . . . . . . . . . 65

6.3 .2 A função de log-verosimilhança . . . . . . . . . . . . . . . . 66

6.3 .3 Função escore . . . . . . . . . . . . . . . . . . . . . . 67

6.3 .4 Matriz de informação de Fisher . . . . . . . . . . . . . . 67

6.4 Estudo de simulação . . . . . . . . . . . . . . . . . . . . . . . . . 67

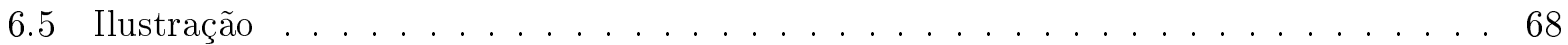

6.5 .1 Dados de vulcões . . . . . . . . . . . . . . . . . 68

6.5 .2 Dados de Engenharia . . . . . . . . . . . . . . . . . . 69

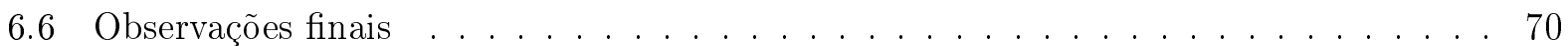

7 Modelo geométrico half-normal potência com fração de cura $\quad 71$

7.1 Motivação . . . . . . . . . . . . . . . . . . . . . . 71

7.2 Formulação do Modelo . . . . . . . . . . . . . . . . . . . . . 71

7.2 .1 Momentos . . . . . . . . . . . . . . . . . 74

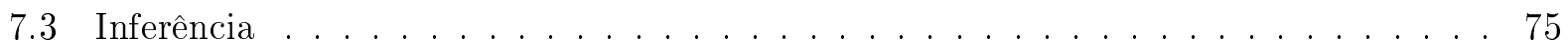

7.4 Estudo de simulação . . . . . . . . . . . . . . . . . . . . . 76

7.5 Ilustração . . . . . . . . . . . . . . . . . . . . . . . . . 76

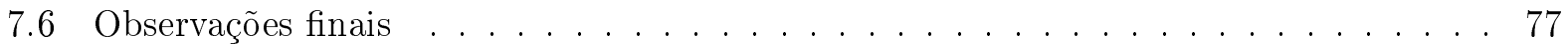

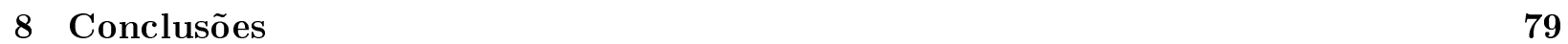

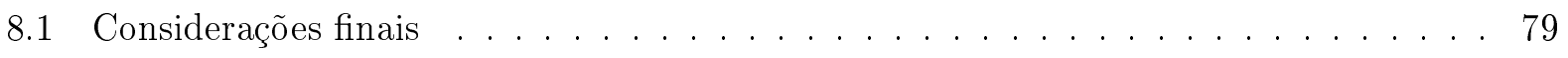

8.2 Sugestões para pesquisas futuras $\ldots \ldots \ldots \ldots \ldots \ldots$

$\begin{array}{ll}\text { A Apêndice } & 81\end{array}$

A.1 Conjunto de Dados. . . . . . . . . . . . . . . . . . . . . 81

Referências Bibliográficas $\quad 83$ 


\section{Lista de Abreviaturas}

f.d.a. função de distribuição acumulada

f.d.p. função de densidade de probabilidade

E.Q.M. erro quadrático médio

i.i.d. independentes e identicamente distribuídos(as)

K-M (estimador de) Kaplan-Meier

EMV estimador de máxima verossimilhança

v.a.('s) variável(variáveis) aleatória(s) 


\section{Lista de Figuras}

2.1 Função de sobrevivência (esquerda) e de risco (direita) para diferentes valores do parâmetro de forma $\alpha$, na distribuição Weibull. . . . . . . . . . . . . . . . . . . 10

2.2 Função de sobrevivência (esquerda) e de risco (direita) do modelo exponencial segmentado

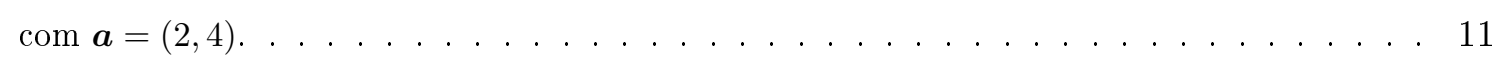

2.3 Função de sobrevivência (esquerda) e de risco (direita) do modelo gamma. . . . . . . . . . 12

2.4 Função de sobrevivência (esquerda) e de risco (direita) para diferentes valores do parâmetro de escala $\sigma$ do modelo log-normal. . . . . . . . . . . . . . . . . . 13

2.5 Função de sobrevivência (esquerda) e de risco (direita) para diferentes valores do parâmetro de escala $\lambda$ do modelo log-logístico. . . . . . . . . . . . . . . . 14

2.6 Função de sobrevivência (esquerda) e de risco (direita) para diferentes valores do parâmetro de escala $\sigma$, da distribuição half-normal. . . . . . . . . . . . . . . 15

2.7 Função de sobrevivência (esquerda) e de risco (direita) para diferentes valores do parâmetro $\alpha$, do modelo Lindley. . . . . . . . . . . . . . . . . . 16

2.8 Função de sobrevivência (esquerda) e de risco (direita) para diferentes valores do parâmetro

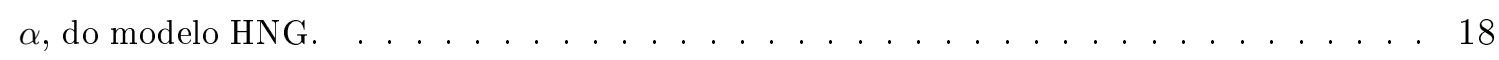

2.9 Função de sobrevivência (esquerda) e de risco (direita) para diferentes valores do parâmetro $\alpha$, do modelo EG. . . . . . . . . . . . . . . . . . . . . . 19

2.10 Função de sobrevivência (esquerda) e de risco (direita) para diferentes valores do parâmetro $\alpha$, do modelo ENH. . . . . . . . . . . . . . . . . . . . . . 20

3.1 Gráficos da função de densidade(esquerda) e função de distribuição acumulada(direita) para $\alpha=1$, e $\beta=0,1,6,20$ do modelo EE. . . . . . . . . . . . . . 24

3.2 Gráficos da função de sobrevivência(esquerda) e função de risco(direita) para $\alpha=1$, e $\beta=0,1,6,20$ do modelo EE. . . . . . . . . . . . . . . . 25

3.3 Algumas características da distribuição EE para diferentes conjuntos dos parâmetros. Painel superior esquerdo: média. Painel superior direito: variância. Painel inferior esquerdo: coeficiente de assimetria. Painel inferior direito: coeficiente de curtose. . . . . . . . . . . 27

3.4 Modelos ajustados com a abordagem de máxima verossimilhança para o conjunto de dados de resistência à ruptura (esquerda) e fda empírica (direita) para o modelo EE. . . . . . . . 31

4.1 Gráfico das funções de densidade e taxa de risco para a distribuição RL. . . . . . . . 35

4.2 Gráfico dos coeficientes de assimetria e curtose para o modelo RL. . . . . . . . . . . 37

4.3 Função de distribuição acumulada para os modelos ajustados aos dados dos tempos de sobrevivência de cobaias. . . . . . . . . . . . . . . . . . . 44

4.4 Graficos de dispersão e boxplot dos dados. . . . . . . . . . . . . . . . . . . . 44 
4.5 Função de distribuição acumulada para os modelos ajustados no conjunto de dados de tempos de falhas. . . . . . . . . . . . . . . . . . . 45

5.1 Função de densidade (esquerda) e função de risco (direita) para uma partição de tempo em que $L=2$ e $a_{1}=2$ para vários valores de $\lambda_{1}, \lambda_{2}$ e $\ldots \ldots \ldots \ldots \ldots \ldots$

5.2 Algumas características da distribuição ESP para valores diferentes dos parâmetros. Painel superior esquerdo: média. Painel superior direito: variância. Painel inferior esquerdo: coeficiente de assimetria. Painel inferior direito: coeficiente de curtose. Em todos os casos $L=2$

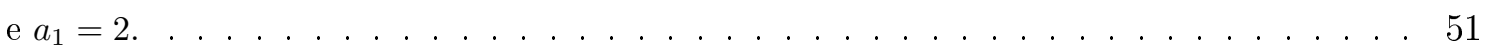

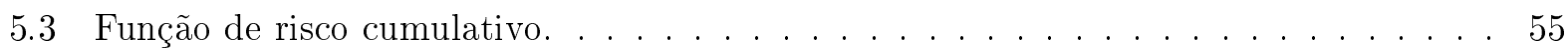

5.4 Função de distribuição empírica e funções de distribuição cumulativa estimada para alguns modelos do conjunto de dados kevlar 49/epoxy (sem o ponto final dos dados). O painel à direita é um destaque. . . . . . . . . . . . . . . . . . . . 57 57

5.5 Função de risco cumulativo estimado para dados do estudo de radioterapia. . . . . . 58

5.6 Função de sobrevivência estimado para os dados do estudo de radioterapia sob o modelo $\operatorname{ESP}(2), \operatorname{ES}(2)$ e WE. . . . . . . . . . . . . . . . . . 59

5.7 Perfil de log-verossimilhança para $\lambda_{1}, \lambda_{2}$ e $\alpha$ no modelo ESP com observações censuradas no conjunto de dados do estudo de radioterapia. . . . . . . . . . . . . . .

6.1 Função de densidade de $T$ para $\sigma=1, \alpha=0.5,0.8,1,1.2,2$. (esquerda) e função de distribução para $\sigma=1$ e $\alpha=0.5,1,1.2,4,10$.(direita) do modelo PHN. . . . . . . . . 62

6.2 Função de sobrevivência $T$ para $\sigma=1$ e $\alpha=0.5,0.8,1,1.2,2$.(direita) e função de risco para $\sigma=1$ e $\alpha=0.5,0.8,1,1.2,2 \ldots \ldots \ldots \ldots \ldots$

6.3 Algumas características da distribuição PHN para um conjunto diferente de parâmetros. Painel superior esquerdo: média. Painel superior direito: variância. Painel inferior esquerdo: coeficiente de assimetria. Painel inferior direito: coeficiente de curtose. . . . . . . . . . . 66

6.4 Histograma para conjuntos de dados de alturas do vulcão, linhas representam distribuições ajustadas utilizando estimadores de máxima verossimilhança(esquerda) e funções de sobrevivência e a sobrevivência empírica(direita) . . . . . . . . . . . . .

6.5 Histograma para conjuntos de dados de engenharia, linhas representam distribuições ajustadas utilizando estimadores de máxima verossimilhança(esquerda) e funções de sobrevivência e a sobrevivência empírica (direita) . . . . . . . . . . . . . . . .

7.1 Gráficos das funções densidade(esquerda) e sobrevivência(direita) da distribuição geométrica half-normal potência para $\sigma=1.5$, e $\alpha=1.5 \ldots \ldots \ldots \ldots$. . . . 73

7.2 Gráficos das funções de risco da distribuição Geométrica Half-Normal Potência para $\sigma=1.5, \alpha=0.5($ esquerda),$\alpha=1.5$ (direita) $\ldots \ldots \ldots \ldots \ldots$

7.3 Gráficos da assimetria da distribuição geométrica half-normal potência (esquerda) e

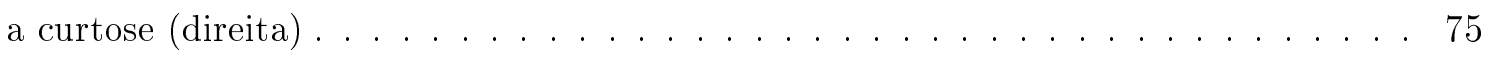

7.4 Estimador de Kaplan-Meier. . . . . . . . . . . . . . . . . . . . . . . . . . 77

7.5 Gráficos dos resíduos do modelo (esquerda), e do ajuste do estimador Kaplan-Meier para os pacientes com ulceração e considerando a media do espessor (direita) . . . . 


\section{Lista de Tabelas}

2.1 Relação entre as funções de interesse na análise de sobrevivência. . . . . . . . . . . 6

2.2 Função de densidade, função de sobrevivência e taxa de falha para algumas distri-

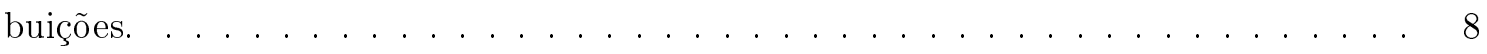

2.3 função de densidade, função de sobrevivência e taxa de falha para algumas distribuições. 17

3.1 Estatísticas descritivas para o conjunto de dados de vida de fratura por fatiga . . . . 30

3.2 As estimativas dos parâmetros para os modelos EG, ENH, Lindley e EE para o conjunto de dados de vida de fratura por fatiga. . . . . . . . . . . . . 30

4.1 Estudo de simulação para os EMV's no modelo RL. . . . . . . . . . . . . . . . . 41

4.2 Estudo de simulação para estimadores de momentos do modelo RL. . . . . . . . . . . 42

4.3 Estatísticas descritivas para os tempos de sobrevivência de cobaias. . . . . . . . . . . 43

4.4 Estimativas dos parâmetros, desvíos padrões(d.p.) e valor da função log-verossimilhança para os modelos $R, W, G$ e $R L$ para os tempos de sobrevivência de cobaias. . . . . . 43

4.5 Testes de bondade de ajustes para o modelo RL . . . . . . . . . . . . . . . 43

4.6 Estatísticas descritivas para o conjunto de dados dos tempos de falha de componentes. 44

4.7 Estimativas dos parâmetros e os valores da log-verossimilhança para os modelos $R$, $W, G$ e $R L$ para o conjunto de dados dos tempos de falha. . . . . . . . . . . . 45

4.8 Testes de bondade de ajuste para o modelos RL para o conjunto de dados dos tempos de falha . . . . . . . . . . . . . . . . . . . . 45

5.1 Médias empíricas e desvios-padrões para estimadores de máxima verossimilhança de

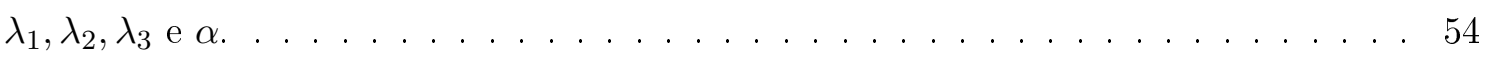

5.2 As estimativas dos parâmetros (com (DP) indicando o desvio padrão) e as distribuições ajustadas para o conjunto de dados completos de kevlar. . . . . . . . . . . 56

5.3 As estimativas dos parâmetros (com (DP), indicando desvio padrão) e distribuições ajustadas (dados kevlar sem observação 101) . . . . . . . . . . . . . . . 57

5.4 Critério de AIC para o conjunto de dados de radioterapia. . . . . . . . . . . . . 58

5.5 Parâmetros estimados para o modelo ESP. . . . . . . . . . . . . . . . . . . 59

6.1 Médias empíricas e desvios padrão para diferentes valores de $\alpha . \ldots \ldots \ldots$. . . . 67

6.2 As estatísticas descritivas para o conjunto de dados das alturas do vulcões . . . . . . 68

6.3 Estimativas dos parâmetros dos modelos HNG e PHN para o conjuntos de dados dos Vulcões e suas correspondentes estatísticas AIC, CAIC e BIC. . . . . . . . . . . . . 68

6.4 Testes de bondade de ajuste. . . . . . . . . . . . . . . . 68

6.5 Estatísticas descritivas para os dados de Engenharia. . . . . . . . . . . . . . . 69 
6.6 Estimativas dos parâmetros do modelo para o conjunto de dado de engenharia e suas correspondentes estatísticas AIC, CAIC e BIC. . . . . . . . . . . . . . . 69

6.7 Testes de bondade de ajuste. . . . . . . . . . . . . . . . . . . . 70

7.1 Momentos para algumas combinações de parâmetros da distribuição GHNP. . . . . . 74

7.2 Médias das estimativas de máxima verossimilhança (EMVs), desvios padrões (DP) e a raiz quadrada dos erros quadraticos médios $(\sqrt{E Q M})$ da fração de cura $p_{0}^{(0)}$ e $p_{0}^{(1)}$ para dados simulados do modelo de fração de cura HNP. . . . . . . . . . . . . . . 77

7.3 Estimação por Máxima Verossimilhança para os paramêtros estimados para os modelos MPHN e GPHNfc. . . . . . . . . . . . . . . . . . . . 78 


\section{Capítulo 1}

\section{Motivação}

\subsection{Introdução}

Extensões de distribuções de probabilidade contínuas têm sido muito estudadas nas últimas décadas. Uma das razões para generalizar uma distribuição conhecida é porque a sua forma generalizada pode flexibilizar a distribuição de tal forma que se possa acomodar formas da função de taxa de falha que sejam não monótonas. Como um exemplo, temos a família das distribuições potência, cuja função de densidade de probabilidade (fdp) é da forma

$$
\varphi_{F}(t ; \alpha)=\alpha f(t)\{F(t)\}^{\alpha-1}, \quad \alpha \in \mathbb{R}^{+},
$$

proposta por Lehmann [1953] no caso de $\alpha$ inteiro ou racional e Durrans [1992] no caso de $\alpha$ real, em que $F(t)$ é a função de distribuição acumulada (fda) e $f(t)$ é a fdp de uma variável aleatória(v.a.) não negativa.

Por essa razão, uma linha de pesquisa importante na área de sobrevivência é propor novas distribuições que permitam caracterizar dados de sobrevivência com função de taxa de falhas monótona, unimodal e na forma de banheira. Desde então, diversos trabalhos com novas distribuições de probabilidade vêm sendo desenvolvidos. Por exemplo, Rogers e Tukey [1972], Mosteller e Tukey [1977], Eugene et al. [2002], Nadarajah e Kotz [2006a] e Pescim et al. [2010], entre outros.

A análise de sobrevivência é uma das áreas da estatística que mais cresceu nas últimas décadas. A razão desse crescimento é a utilização de técnicas em conjunto com computadores cada vez mais eficientes que permitem conduzir análise de dados, levando em conta a informação de observações censuradas (Colosimo e Giolo [2006]). No contexto de análise de sobrevivência, as distribuições clássicas associadas à variável resposta mais usuais são a distribuição exponencial, a distribuição Weibull, a distribuição log-normal e a distribuição log-logística.

Contudo, em algumas situações práticas, Aarset [1987] e Mudholkar et al. [1996] verificaram que a função de taxa de falha pode assumir formas não monótonas, ou seja, na forma de banheira ou unimodal. Nesse caso, a distribuição Weibull e a distribuição exponencial não podem ser utilizadas, pois não apresentam essa característica. Por outro lado, a distribuição log-normal e a distribuição log-logística são apropriadas apenas em situações nas quais a função de taxa de falhas é unimodal.

Por essa razão, generalizar distribuições de probabilidade contínuas tem sido uma proposta enfatizada na literatura, com a finalidade de fornecer maior flexibilidade às distribuições transformadas e permitir que a função de taxa de falha possa descrever formas do tipo monótona, unimodal e de banheira. Além disso, a distribuição modificada pode ser utilizada para discriminação entre modelos, pois possui como caso particular a distribuição a qual lhe deu origem (Hashimoto et al. [2013]). 


\subsection{Objetivos}

O objetivo geral desta tese é estender diversos modelos de distribuições com suporte positivo, mediante técnicas descritas na literatura(como, por exemplo, a composição de distribuições) para obter distribuições com maior flexibilidade. Com este objetivo em mente apresentamos a seguir as principais metas para esta tese:

- Propór uma extensão do modelo exponencial, com a finalidade de que a função de risco, seja não monótona, e seja uma alternativa para o modelo exponencial generalizado, proposto por Gupta e Kundu [1999] e para a extensão do modelo exponencial proposta por Nadarajah e Haghighi [2011];

- Propór uma extensão do modelo half-normal, com a finalidade de flexibilizar a função de risco, e que seja uma alternativa ao modelo half-normal generalizado proposto por Cooray e Ananda [2008];

- Estender o modelo exponencial segmentado desenvolvido por Friedman [1982] com o objetivo de flexibilizar a função de risco, que é constante em cada um dos intervalos da partição considerada;

- Desenvolver um modelo (denominado Rayleigh/Lindley), que resulta da composição das distribuições Rayleigh e Lindley discreta;

- Considerar em alguns dos modelos antes mencionados a presença de censura;

- Considerar um contexto de regressão para alguns dos modelos antes propostos.

\subsection{Organização da Tese}

A tese de doutorado envolve os seguintes capítulos:

- Capítulo 2: Apresentamos algumas distribuições importantes desenvolvidas na literatura que dão suporte aos desenvolvimentos desta tese.

- Capítulo 3: Consideramos uma extensão da distribuição exponencial com base em misturas de distribuições positivas. Estudamos as principais propriedades desta nova distribuição, com ênfase especial em seus momentos, função geradora de momentos e algumas características relacionadas aos estudos de confiabilidade e análise de sobrevivência. Discutimos também a estimação de parâmetros considerando as abordagens por máxima verossimilhança e momentos. Finalmente, ajustamos o modelo desenvolvido a um conjunto de dados reais.

- Capítulo 4: Neste capítulo é introduzida a distribuição Rayleigh-Lindley (RL), que é obtida compondo as distribuições Rayleigh e Lindley discreta, em que o procedimento de composição segue a mesma forma que a utilizada por Adamidis e Loukas [1998]. A distribuição resultante é um modelo de dois parâmetros competitivo com outros modelos parsimoniosos como as distribuições gama e Weibull. Estudamos algumas propriedades deste novo modelo como os momentos e a vida média residual. A estimação dos parâmetros foi abordada através dos métodos de máxima verossimilhança via algoritmo EM e os estimadores de momentos. O comportamento desses estimadores foi estudado em um estudo de simulação. Finalmente, são descritas duas ilustrações com dados reais, a fim de mostrar o desempenho do modelo frente a outros dois modelos de dois parâmetros.

- Capítulo 5: Propomos uma extensão da distribuição exponencial segmentada. Propriedades de sua função densidade e função de risco são investigadas. Inferência por máxima verosimilhança é discutida e a matriz de informação de Fisher é obtida. Os resultados de duas aplicações com dados reais são relatadas, em que o modelo é ajustado usando máxima verosimilhança. As aplicações ilustram o melhor desempenho da nova distribuição quando comparada com outros modelos alternativos, propostos recentemente na literatura. 
- Capítulo 6: Propomos uma extensão da distribuição half-normal com base na distribuição do máximo de uma amostra aleatória. Mostra-se que esta distribuição pertence à família de distribuições beta half-normal generalizada. Propriedades de sua função densidade são investigadas, estimadores de máxima verossimilhança são discutidos e a matriz de informação de Fisher é derivada para a situação de dados completos. Na presença de censuras, usa-se-a a matriz de informação observada. Uma ilustração com dados reais é apresentada e comparações com extensões alternativas da distribuição half-normal serão investigadas.

- Capítulo 7: Será apresentado o modelo proposto acima, no contexto de modelos de longa duração, isto é, adicionado um parámetro associado à cura, considerando a distribuição geométrica para o número de causas iniciais proposto por Rodrigues et al. [2009]. Procuramos também desenvolver estudos de simulação e uma aplicação com dados reais.

- Capítulo 8: Apresentamos as principais conclusões e contribuições desta tese. Apresentamos também algumas direções para pesquisas futuras.

Parte deste trabalho já foi aceito em periódicos de circulação internacional (Gómez et al. [2014], Gómez e Bolfarine [2015] e Gómez e Bolfarine [2016]). 


\section{Capítulo 2}

\section{Preliminares}

\subsection{Conceitos básicos em análise de sobrevivência}

\subsubsection{Função de sobrevivência}

A função de sobrevivência corresponde à probabilidade de um indivíduo sobreviver além do tempo $t$ (experimentando o evento após o tempo $t$ ) é definida como

$$
S(t)=\mathbb{P}(T>t)=\int_{t}^{\infty} f(x) d x
$$

em que $T$ é a variável aleatória que representa o tempo de falha. Note que $f(t)=-\frac{\partial S(t)}{\partial t}$

No contexto de falhas de equipamento ou de um artigo fabricado em uma linha de produção, $S(t)$ representa a função de confiabilidade. Se $T$ é uma variável aleatória contínua, segue que $S(t)$ é uma função contínua, estritamente decrescente. A função de sobrevivência é o complemento da função de distribuição cumulativa, isto é, $S(t)=1-F(t)$, em que $F(t)=\mathbb{P}(T \leqslant t), t \in(-\infty, \infty)$.

Muitos tipos de funções de risco podem ser consideradas, mas elas tem as mesmas propriedades básicas. Elas são funções monótonas não crescentes do tempo t quando este se aproxima de infinito. A sua taxa de declínio, naturalmente, varia de acordo com o risco de sofrer o evento no tempo $t$, mas é difícil determinar a essência de um padrão de falhas olhando simplesmente para a curva de sobrevivência. No entanto, esta quantidade continua a ser uma descrição popular de sobrevivência na literatura aplicada e pode ser muito útil para a comparação de dois ou mais padrões de mortalidade.

\subsubsection{Função de risco ou função de taxa de falha}

A quantidade básica, fundamental na análise de sobrevivência, é a função de risco. Esta função também é conhecida como a taxa condicional de falha em termos de confiabilidade, a força de mortalidade em demografia, a função de intensidade em processos estocásticos, a taxa de falha específica à idade em epidemiologia, ou simplesmente como a taxa de risco. A função de risco é definida por

$$
h(t)=\lim _{\Delta t \rightarrow 0} \frac{\mathbb{P}(t \leq T<t+\Delta t \mid T \geq t)}{\Delta t} .
$$

Se $T$ é uma variável aleatória contínua, pode-se verificar que

$$
h(t)=\frac{f(t)}{S(t)}=\frac{-\partial \log (S(t))}{\partial t} .
$$

Uma quantidade relacionada é a função de risco acumulada $H(t)$, definida por

$$
H(t)=\int_{0}^{t} h(u) d u=-\log (S(t))
$$

Assim, chega-se ao resultado

$$
S(t)=\exp (-H(t))=\exp \left(-\int_{0}^{t} h(u) d u\right)
$$




\begin{tabular}{ccccc} 
& \multicolumn{4}{c}{ Função obtida } \\
\cline { 2 - 4 } Função requerida & $f(t)$ & $S(t)$ & $h(t)$ & $H(t)$ \\
\hline$f(t)$ & - & $\int_{t}^{\infty} f(u) d u$ & $\frac{f(t)}{\int_{t}^{\infty} f(u) d u}$ & $-\log \left(\int_{t}^{\infty} f(u) d u\right)$ \\
$S(t)$ & $-\frac{d S(t)}{d t}$ & $-\frac{[d S(t) / d t]}{S(t)}$ & $-\log S(t)$ \\
$h(t)$ & $h(t) \exp \left\{-\int_{0}^{t} h(u) d u\right\}$ & $\exp \left\{-\int_{0}^{t} h(u) d u\right\}$ & - & $\int_{0}^{t} h(u) d u$ \\
$H(t)$ & $\frac{d H(t)}{d t} \exp \{-H(t)\}$ & $\exp \{-H(t)\}$ & $\frac{d H(t)}{d t}$ & -
\end{tabular}

Tabela 2.1: Relação entre as funções de interesse na análise de sobrevivência.

A partir da expressão (2.1) pode-se ver que $h(t) \Delta t$ pode ser vista como a probabilidade aproximada de um indivíduo com idade igual a $t$ unidades de tempo experimentar o evento no instante seguinte. Esta função é particularmente útil para determinar as distribuições das taxas de falhas adequadas utilizando informação qualitativa sobre o mecanismo de falhas e para descrever o modo como a possibilidade de experimentar o evento muda com o tempo. Existem muitas formas gerais para a taxa de risco. A única restrição sobre $h(t)$ é ser não negativa, isto é, $h(t) \geq 0, t>0$. Por exemplo, pode-se acreditar que a taxa de risco para a ocorrência de um evento em particular está aumentando, diminuindo, ser em forma de banheira ou constante ou ainda, unimodal. Modelos com o aumento das taxa de risco podem surgir quando existe envelhecimento natural ou desgaste. Funções de risco decrescentes são muito menos comuns, mas podem ser encontradas ocasionalmente quando há uma probabilidade muito precoce de falha, como em certos tipos de dispositivos eletrônicos ou em doentes com determinados tipos de transplantes. Na maioria das vezes, funções de risco em forma de banheira podem ser apropriadas em populações acompanhadas desde o nascimento. Da mesma forma, alguns equipamentos fabricados podem experimentar falha prematura devido a peças defeituosas, seguido por uma taxa de risco constante que, nas fases posteriores da vida útil do equipamento, aumenta. A maioria dos dados de mortalidade da população segue este tipo de função de risco, em que, durante um breve período, as mortes resultam, principalmente, de doenças infantis, após a qual a taxa de mortalidade estabiliza, seguido de um aumento da taxa de risco devido ao processo natural de envelhecimento. Finalmente, se a taxa de risco aumenta no início e, eventualmente, começa a diminuir, é denominada em forma de "côncavo". Este tipo de taxa de risco é frequentemente usado em modelagem da sobrevivência após uma cirurgia bem sucedida em que há um aumento inicial em risco devido a infecção, hemorragia, ou outras complicações apenas após o procedimento, seguido por um declínio constante em risco quando o paciente se recupera (Klein e Moeschberger [2003]).

Notamos que existe uma relação biunívoca entre as funções $f(t), S(t), h(t)$ e $H(t)$ discutidas anteriormente. Desta forma, dada uma delas pode-se encontrar todas as outras de forma única. A Tabela 2.1 apresenta a relação entre as funções mencionadas anteriormente. Distribuições específicas que dão origem a esses diferentes tipos de taxas de falhas são apresentadas a seguir. 


\subsection{Principais distribuições com suporte positivo}

Alguns dos modelos importantes a serem considerados incluem os modelos exponencial, Weibull, log-normal, log-logística, normal, Rayleigh, half-normal, Lindley e exponencial segmentada . As suas funções de sobrevivência, as taxas de risco, as funções densidade e tempos de vida esperados estão resumidos na Tabela 2.2 . 


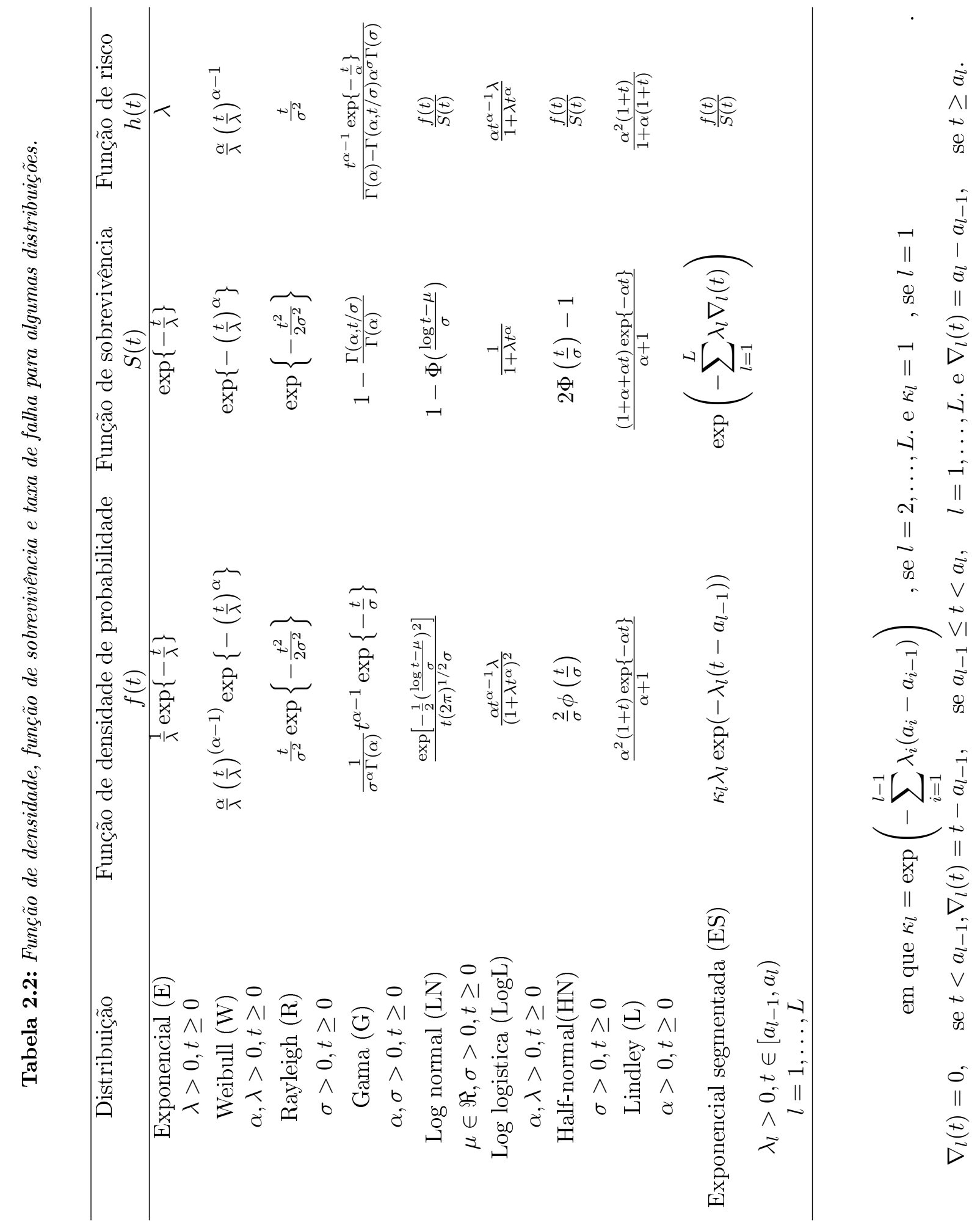


Devido a importância histórica, simplicidade matemática e propriedades importantes, vamos discutir em primeiro lugar, a distribuição exponencial. Ela é caracterizada por ter sua função de taxa de falha constante, isto é, $h(t ; \lambda)=\frac{1}{\lambda}$. A função de densidade de probabilidade para a variável aleatória, $T$, com distribuição exponencial é dada por

$$
f(t ; \lambda)=\frac{1}{\lambda} \exp \left(-\frac{t}{\lambda}\right), \quad t \geq 0
$$

em que o parâmetro $\lambda$ tem a mesma unidade que o tempo de falha, $t$. Isto é, se $t$ é medido em horas, $\lambda$ também será medido em horas. A distribuição exponencial possui as seguintes propriedades. A primeira, referida como a falta de memória, é dada por

$$
\mathbb{P}(T \geq t+z \mid T>t)=\mathbb{P}(T \geq z)
$$

que permite a sua tratabilidade matemática, mas também reduz a sua aplicabilidade para muitas situações reais. Devido a esta propriedade distributiva, segue-se que $\mathbb{E}(T-t \mid T>t)=\mathbb{E}(T)=\lambda$; isto é, a vida média residual é constante. Isso ocorre porque o tempo até a ocorrência futura de um evento não depende da história passada. Essa propriedade é às vezes chamada de propriedade de "não-envelhecimento" ou a propiedade "de tão velho como novo". Essa propriedade também é refletida na taxa de falha constante da distribuição exponencial. Aqui, a probabilidade condicional de falha em qualquer instante $t$, uma vez que o evento não ocorreu antes do tempo $t$, não depende de $t$. Embora a distribuição exponencial tem sido historicamente muito popular, sua taxa de falha constante parece demasiado restritiva, tanto em aplicações industriais quanto para as de saúde.

Outra distribuição popular é a distribuição de Rayleigh, homenagem ao físico e matemático inglês John William Strutt, conhecido como o terceiro barão de Rayleigh, é uma distribuição de probabilidade contínua para valores positivos. Geralmente, essa distribuição é observada quando a magnitude global de um vetor está relacionada com os seus componentes de direção. Por exemplo, quando a velocidade do vento é analisada em duas direções, ou seja, um vetor de componentes bidimensional. Assumindo que as componentes são independentes e normalmente distribuídas com média zero e variâncias iguais, então a velocidade geral do vento tem um a distribuição de Rayleigh. Veja Siddiqui [1964] para mais detalhes. Um segundo exemplo da distribuição surge no caso de números complexos aleatórios cujos componentes real e imaginário são independentemente e identicamente distribuídos Gaussiana com média zero e variâncias iguais. Nesse caso, o valor absoluto do número complexo é distribuído Rayleigh.

Devido à distribuição exponencial e Rayleigh ser casos especiais da distribuição Weibull, consideramos os parágrafos subseqüentes para revisar algumas outras propriedades que estão implícitas na discussão desta distribuição. Rosin e Rammler [1933] a usaram para descrever as "leis que regem a finura do carvão em pó", e Weibull[1939, 1951], propôs a mesma distribuição, que mais tarde recebeu seu nome para descrever a duração de vida dos materiais. Desde então, a mesma vem sendo frequentemente usada em estudos biomédicos e industriais. Sua função de sobrevivência é dada por $S(t ; \alpha, \lambda)=\exp \left\{-\left(\frac{t}{\lambda}\right)^{\alpha}\right\}$, para $t>0$. Aqui $\lambda>0$ é o parâmetro de escala, e $\alpha>0$ é o parâmetro de forma. Quando $\alpha=1$, tem-se a distribuição exponencial e, logo a distribuição exponencial é um caso particular da distribuição de Weibull. Figura 2.1, apresenta uma variedade de funções de sobrevivência de Weibull. A sua função de risco tem uma forma flexível dada por

$$
h(t ; \alpha, \lambda)=\frac{\alpha}{\lambda}\left(\frac{t}{\lambda}\right)^{\alpha-1}
$$

Pode-se ver a partir da Figura 2.1 que a distribuição Weibull é flexível o suficiente para acomodar taxas de falhas crescentes $(\alpha>1)$, decrescentes $(\alpha<1)$, ou taxas de falhas constantes $(\alpha=1)$. Este fato, juntamente com as expressões das funções de sobrevivência, risco, e a densidade de probabilidade são quantidades relativamente simples e de fácil interpretação que tornaram o modelo muito popular na literatura estatística. É evidente que a forma da distribuição Weibull depende do 

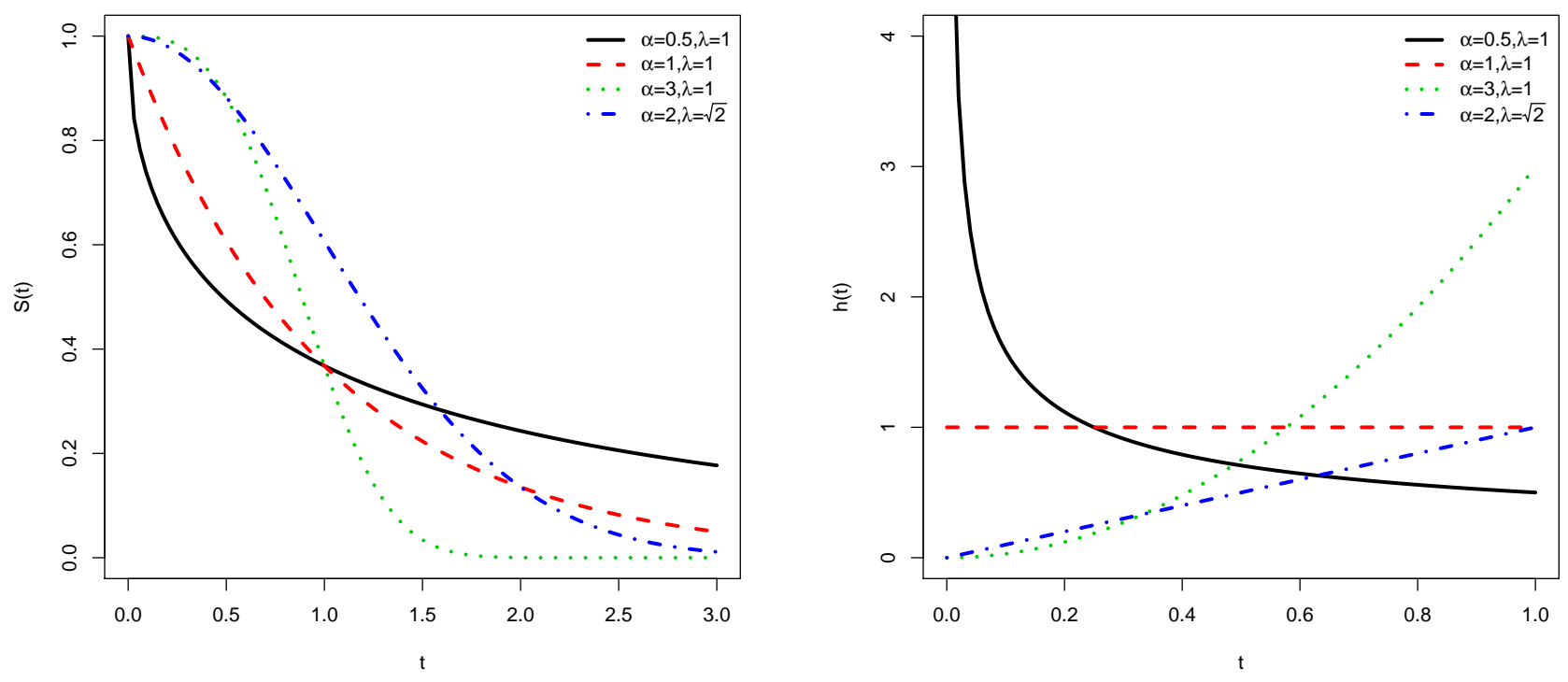

Figura 2.1: Função de sobrevivência (esquerda) e de risco (direita) para diferentes valores do parâmetro de forma $\alpha$, na distribuição Weibull.

valor de $\alpha$. Esta é a razão para referir-se a este parâmetro como o parâmetro de "forma".

A distribuição de Weibull é uma generalização da distribuição de Rayleigh. Neste exemplo, o parâmetro $\sigma$ está relacionado com o parâmetro de escala Weibull $\lambda: \lambda=\sigma \sqrt{2}$ e $\alpha=2$. Pode-se ver na Figura 2.1 na linha em azul, para o parâmetro $\sigma=1$, ou, $\lambda=\sqrt{2}$.

A distribuição exponencial segmentada foi introduzida por Feigl e Zelen [1965]. Subseqüentemente, Friedman [1982] estendeu este modelo com a inclusão de variáveis auxiliares. O modelo de Feigl e Zelen [1965] assume que a função de risco é constante em cada um de um conjunto finito de intervalos de tempo. Uma partição conhecida dos tempos $\boldsymbol{a}=\left(a_{0}, a_{1}, \ldots, a_{L}\right)^{\top}$ de tal modo que $0=a_{0}<a_{1}<\ldots<a_{L-1}<a_{L}=\infty$ é considerada. A variável $T$ é dita ter tem uma distribuição exponencial segmentada com vetor de parâmetros $\boldsymbol{\lambda}=\left(\lambda_{1}, \ldots, \lambda_{L}\right)^{\top}$ e partição $\boldsymbol{a}$ (denotado por $T \sim E S(\boldsymbol{\lambda}, \boldsymbol{a}))$ se

$$
h(t ; \boldsymbol{\lambda})=\lambda_{l}, \quad t \in\left[a_{l-1}, a_{l}\right), \quad l=1, \ldots, L .
$$

As funções de sobrevivência e de densidade correspondentes são dadas por

$$
\begin{gathered}
S(t ; \boldsymbol{\lambda})=\exp \left(-\sum_{l=1}^{L} \lambda_{l} \nabla_{l}(t)\right), \quad t>0 \mathrm{e} \\
f(t ; \boldsymbol{\lambda})=\kappa_{l} \lambda_{l} \exp \left(-\lambda_{l}\left(t-a_{l-1}\right)\right), \quad t \in\left[a_{l-1}, a_{l}\right), \quad l=1, \ldots, L,
\end{gathered}
$$

com

$$
\kappa_{l}= \begin{cases}1 & , \text { se } l=1, \\ \exp \left(-\sum_{i=1}^{l-1} \lambda_{i}\left(a_{i}-a_{i-1}\right)\right) & , \text { se } l=2, \ldots, L .\end{cases}
$$


$\mathrm{e}$

$$
\nabla_{l}(t)= \begin{cases}0, & \text { se } t<a_{l-1}, \\ t-a_{l-1}, & \text { se } a_{l-1} \leq t<a_{l}, \quad l=1, \ldots, L \\ a_{l}-a_{l-1}, & \text { se } t \geq a_{l}\end{cases}
$$
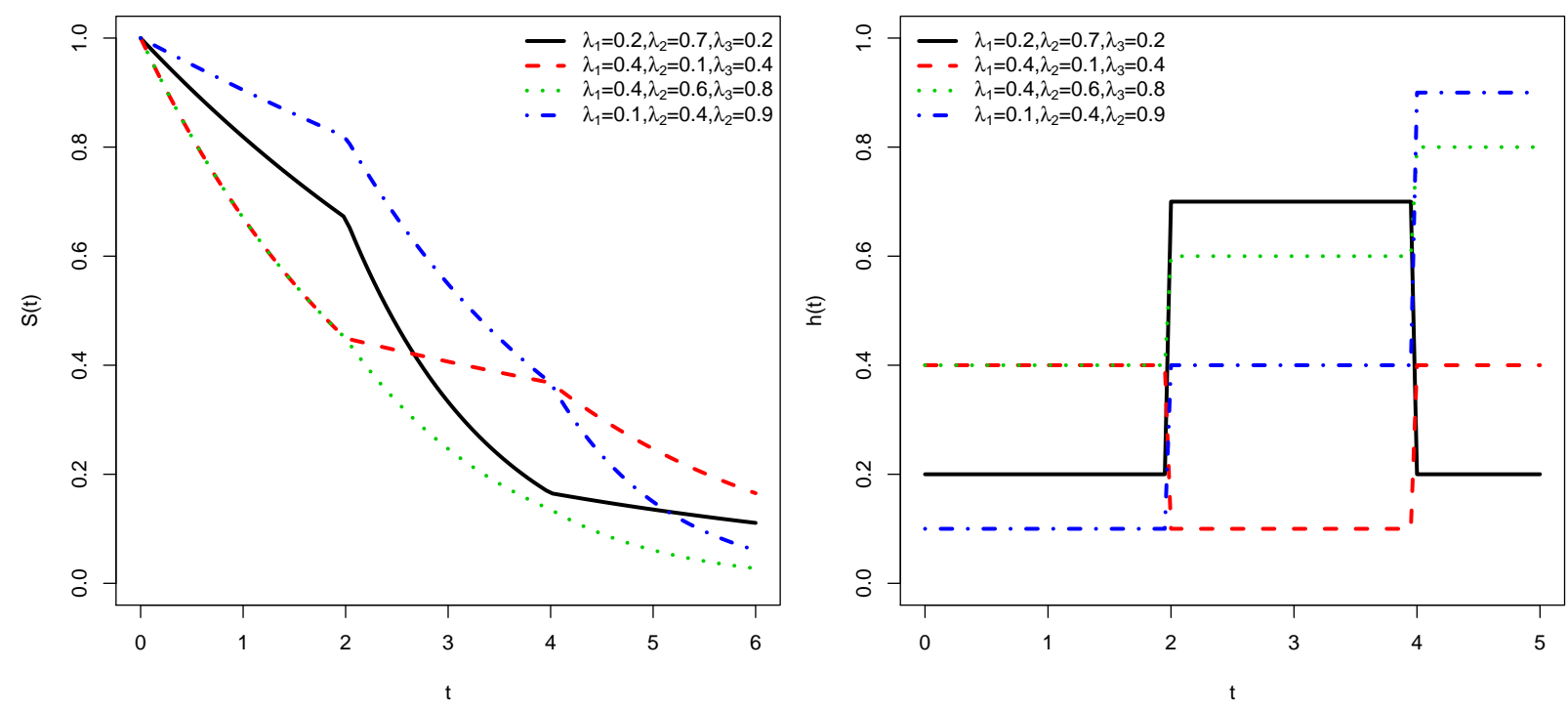

Figura 2.2: Função de sobrevivência (esquerda) e de risco (direita) do modelo exponencial segmentado com $\boldsymbol{a}=(2,4)$.

A distribuição exponencial de parâmetro $\lambda$ é o caso particular da distribuição exponencial segmentada quando $\lambda_{l}=\lambda$, para $l=1, \ldots, L$. Os comportamentos das funções de sobrevivência e de risco estão representadas na Figura 2.2. Na Figura 2.2 pode-se verificar que a função de risco da distribuição exponencial segmentada não é contínua nos pontos do vetor $\boldsymbol{a}$. Isso implica que o conhecimento do vetor $\boldsymbol{a}$ é muito importante. Porém, esta distribuição permite a modelagem de eventos em que a função de risco não é monótona (crescente, decrescente ou constante) como visto na Figura 2.2 no caso em que $\lambda_{1}=0.4, \lambda_{2}=0.1, \lambda=0.4$ e a principal diferença com a Weibull é que a esta última distribuição é adequada só nos casos de eventos com função de risco monótona.

Na prática, o uso desta distribuição também está associado com o uso de uma abordagem semiparamétrica e Bayesiana. Por exemplo, veja-se Pan e Kozakai [2013] e Demarqui et al. [2008].

A distribuição gamma pode ser usada para modelar tempos de serviço, tempo de vida de objetos e tempos de reparo. Uma variável aleatória gamma $T$ com parâmetro de escala $\sigma$ e parâmetro de forma $\alpha$ tem função de densidade de probabilidade

$$
f(t ; \alpha, \sigma)=\frac{1}{\sigma^{\alpha} \Gamma(\alpha)} t^{\alpha-1} \exp \left\{-\frac{t}{\sigma}\right\}, t>0 .
$$

A função de sobrevivência é dada por

$$
S(t ; \alpha, \sigma)=1-\frac{\Gamma(\alpha, t / \sigma)}{\Gamma(\alpha)}
$$


em que $\Gamma(s, t)$ representa a função gamma incompleta superior, definida como

$$
\Gamma(s, t)=\int_{0}^{t} x^{s-1} \exp (-x) d x, s>0 \text { e } t>0
$$

e a função gama é definida como $\Gamma(s)=\Gamma(s, \infty)$.

A função de risco desta distribuição é dada por

$$
h(t ; \alpha, \sigma)=\frac{t^{\alpha-1} \exp \left(-\frac{t}{\alpha}\right)}{\Gamma(\alpha)-\Gamma(\alpha, t / \sigma) \alpha^{\sigma} \Gamma(\sigma)} .
$$

A funções de sobrevivência e de risco, com algumas combinações de parâmetros são ilustradas na Figura 2.3.
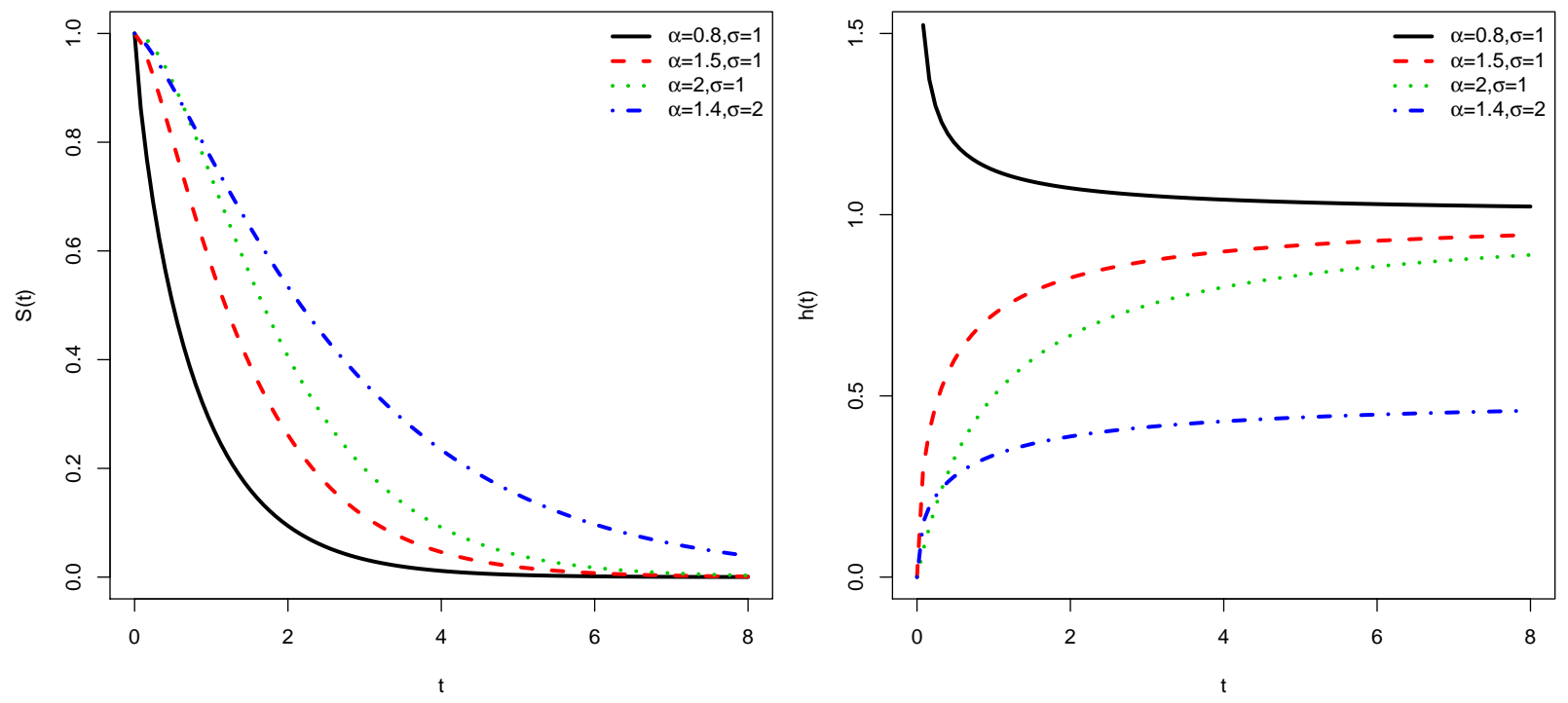

Figura 2.3: Função de sobrevivência (esquerda) e de risco (direita) do modelo gamma.

Por outro lado, uma variável aleatória $T$ segue uma distribuição log-normal se o seu logaritmo $Y=\log T$ segue a distribuição normal. Assim como a distribuição de Weibull, a distribuição lognormal é muito utilizada para caracterizar tempos de vida de produtos e indivíduos. Isto inclui fadiga de metais, semiconductores, diodos e isolamento elétrico. Ela também é bastante utilizada para descrever situações clínicas, como o tempo de vida de pacientes com leucemia. Para dados que dependem do tempo, esta distribuição foi popularizada por sua relação com a distribuição normal (amplamente conhecida na literatura) e porque alguns autores observaram que a distribuição lognormal é uma boa aproximação para a distribuição dos tempos de sobrevivência ou para os tempos de aparecimento de determinadas doenças (Feinleib [1960] e Horner [1987]). Como a distribuição normal, a distribuição log-normal está especificada por dois parâmetros, $\mu$ e $\sigma$, a média e variância de $Y$. Sua função densidade é expressa por

$$
f(t ; \mu, \sigma)=\frac{\phi\left(\frac{\log t-\mu}{\sigma}\right)}{t}=\frac{\exp \left[-\frac{1}{2}\left(\frac{\log t-\mu}{\sigma}\right)^{2}\right]}{t(2 \pi)^{(1 / 2)} \sigma}, \quad t>0, \mu \in \Re, \sigma>0 .
$$

e a sua função de sobrevivência é dada por

$$
S(t ; \mu, \sigma)=1-\Phi\left(\frac{\log t-\mu}{\sigma}\right),
$$



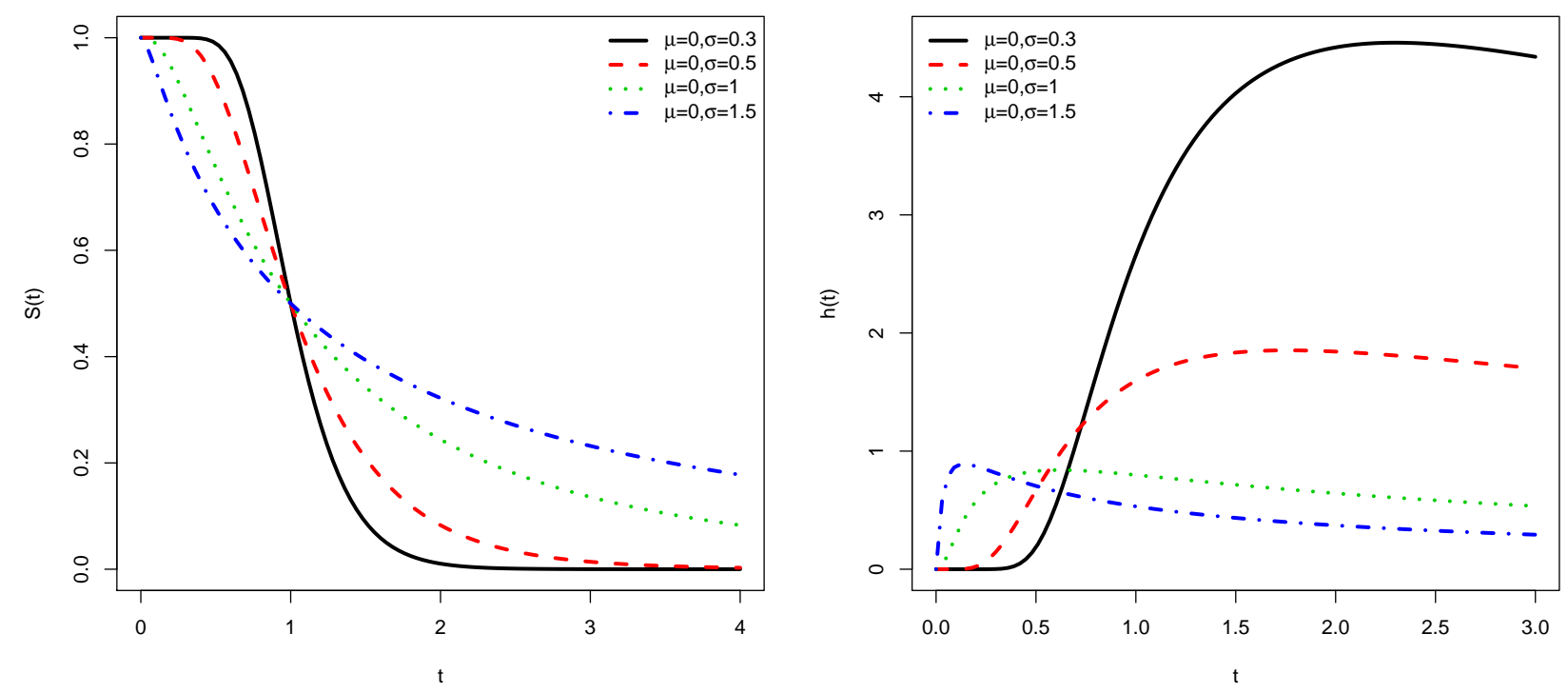

Figura 2.4: Função de sobrevivência (esquerda) e de risco (direita) para diferentes valores do parâmetro de escala $\sigma$ do modelo log-normal.

em que $\phi(\cdot)$ e $\Phi(\cdot)$ são a fda e a fdp da distribuição normal padrão, respectivamente.

A taxa de risco da distribuição é unimodal, isto é, o seu valor inicial é zero, aumenta até atingir um máximo e, em seguida, diminui para zero quando $t$ se aproxima do infinito (veja a Figura 2.4). Este modelo tem sido criticado como uma distribuição de tempos de vida, porque a função de risco está diminuindo para $t$ grande o que parece implausível em muitas situações.

Uma variável aleatória $T$ tem distribuição $\log$-logística se o seu $\operatorname{logaritmo} Y=\log T$ segue a distribuição logística, uma distribuição bastante semelhante a distribuição normal, mas sua função de sobrevivência é matematicamente mais tratável. A função de densidade para $Y$ é expressa por

$$
\frac{\exp \left(\frac{y-\mu}{\sigma}\right)}{\sigma\left[1+\exp \left(\frac{y-\mu}{\sigma}\right)\right]}, \quad-\infty<y<\infty
$$

em que $\mu$ e $\sigma^{2}$ são, respectivamente, o parâmetro de locação e escala de $Y$. Podemos escrever esta distribuição no formato de modelo linear, tomando $Y=\mu+\sigma W$, em que $W$ segue a distribuição logística padronizada com $\mu=0$ e $\sigma=1$.

A taxa de risco e a função de sobrevivência, respectivamente, para a distribuição log-logística podem ser escritas usando expressões relativamente simples

e

$$
h(t ; \alpha, \lambda)=\frac{\alpha \lambda t^{\alpha-1}}{1+\lambda t^{\alpha}}
$$

$$
S(t ; \alpha, \lambda)=\frac{1}{1+\lambda t^{\alpha}}, \quad t>0
$$

em que $\alpha=1 / \sigma>0$ e $\lambda=\exp (\mu / \sigma)$.

O numerador da função de risco é o mesmo o da função de risco da Weibull, mas o denominador faz com que a função de risco assuma as seguintes características: monótona decrescente para $\alpha \leq 1$. Para $\alpha>1$, a função de risco aumenta inicialmente para um máximo de $[(\alpha-1) / \lambda]^{1 / \alpha}$ e, em seguida, decresce para zero quando o tempo se aproxima do infinito, como mostrado na Figura 2.5.

Esta distribuição tem similaridades com as distribuições Weibull e exponencial pelas formas 

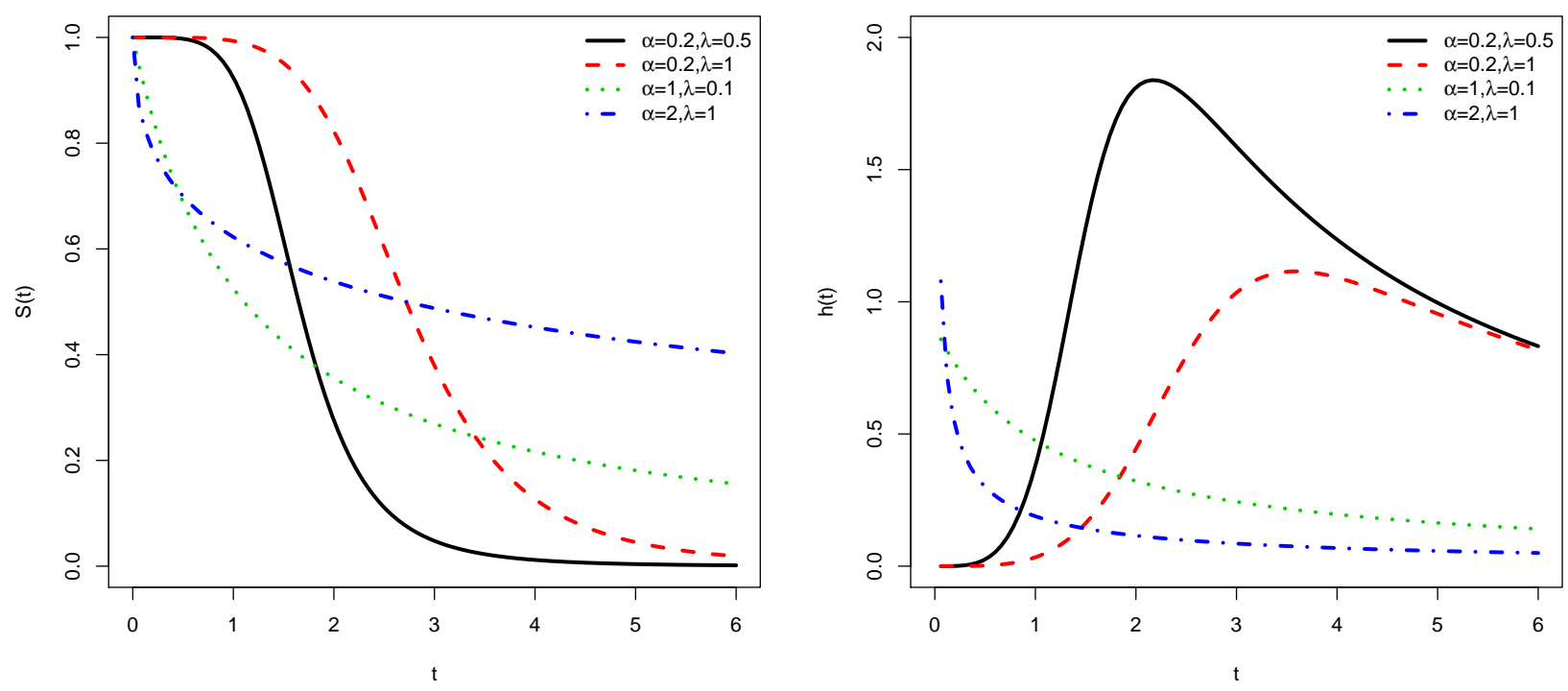

Figura 2.5: Função de sobrevivência (esquerda) e de risco (direita) para diferentes valores do parâmetro de escala $\lambda$ do modelo log-logístico.

fechadas das funções $h(t)$ e $S(t)$. A sua taxa de risco é semelhante a da log-normal, exceto nas caudas, mas tem a vantagem de que a sua função de risco é mais simples.

A distribuição half-normal é muito importante na área das distribuições skew-simétricas, uma vez que é utilizada na representação estócastica do modelo "skew"-normal, estudado por Azzalini[1985,1986], Henze [1986], entre outros. Nos últimos anos, essa distribuição foi usada para modelar dados positivos e está se tornando um importante modelo na teoria de confiabilidade. Assim, o interesse está focado no estudo do parâmetro de escala. Sua função de densidade, é

$$
f(t ; \sigma)=\frac{2}{\sigma} \phi\left(\frac{t}{\sigma}\right)=\frac{2}{\sqrt{2 \pi} \sigma} \exp \left(-\frac{t^{2}}{2 \sigma^{2}}\right), \quad t>0,
$$

em que $\sigma$ é o parâmetro de escala. A taxa de risco e função de sobrevivência, respectivamente, são

$$
h(t ; \sigma)=\frac{\phi\left(\frac{t}{\sigma}\right)}{\sigma\left(1-\Phi\left(\frac{t}{\sigma}\right)\right)}
$$

e

$$
S(t ; \sigma)=2\left(1-\Phi\left(\frac{t}{\sigma}\right)\right)
$$

Os comportamentos das funções de sobrevivência e de risco estão representadas na Figura 2.6.

Finalmente, a distribuição de Lindley foi originalmente proposta por Lindley [1958] no contexto das estatísticas fiduciais e Bayesianas, com a seguinte fdp

$$
\begin{aligned}
f(t ; \alpha) & =\frac{\alpha^{2}}{\alpha+1}(1+t) \exp \{-\alpha t\}, \quad t>0, \alpha>0 \\
& =p \psi_{1}(t)+(1-p) \psi_{2}(t)
\end{aligned}
$$

em que

$$
p=\frac{\alpha}{\alpha+1}
$$



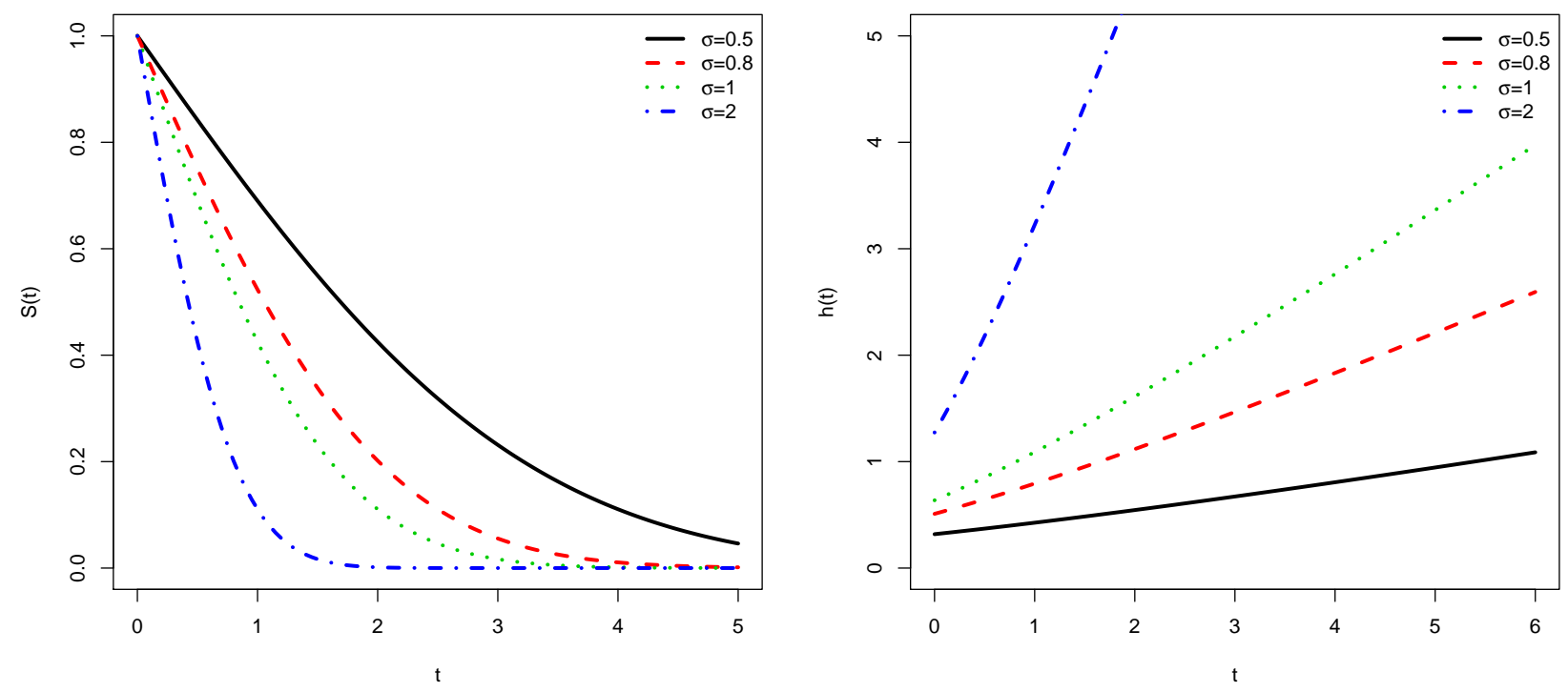

Figura 2.6: Função de sobrevivência (esquerda) e de risco (direita) para diferentes valores do parâmetro de escala $\sigma$, da distribuição half-normal.

$$
\psi_{1}(t)=\alpha \exp \{-\alpha t\}, t>0,
$$

e

$$
\psi_{2}(t)=\alpha^{2} \exp \{-\alpha t\}, t>0 .
$$

A fdp em (2.18) revela que a distribuição Lindley é uma mistura de dois componentes de uma distribuição exponencial(com escala $\alpha$ ) e uma distribuição gamma (com forma 2 e escala $\alpha$ ), com proporção de mistura $p=\alpha /(\alpha+1)$. Os comportamentos das funções de sobrevivência e de risco estão representadas na Figura 2.7.

\subsection{Algumas extensões de distribuições conhecidas}

Nesta subseção apresentamos algumas extensões de distribuições apresentadas na subseção anterior propostas na literatura e que serão utilizadas nos próximos capítulos. 

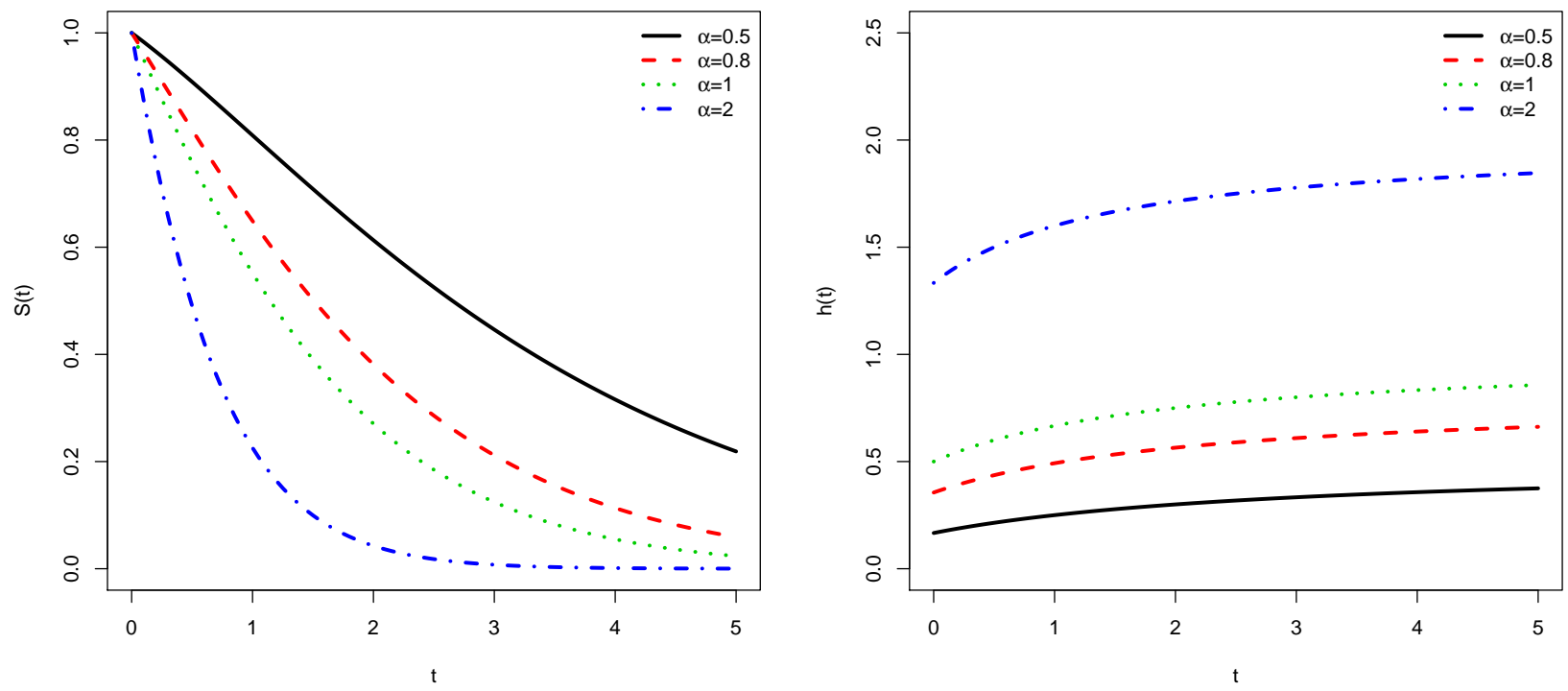

Figura 2.7: Função de sobrevivência (esquerda) e de risco (direita) para diferentes valores do parâmetro $\alpha$, do modelo Lindley. 


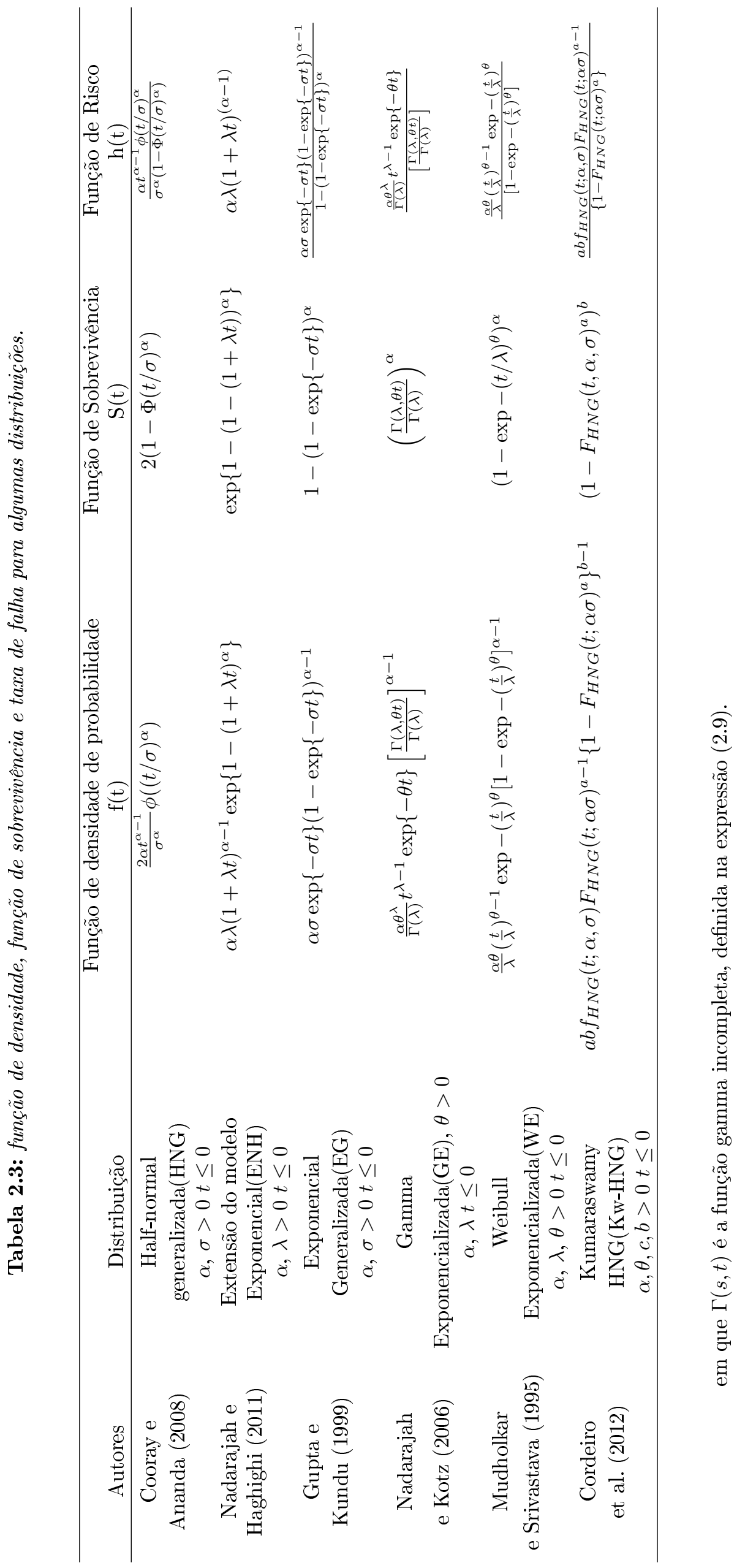




\subsubsection{Half-normal generalizada [Cooray e Ananda [2008]]}

A distribuição half-normal generalizada foi proposta por Cooray e Ananda [2008] como uma alternativa a alguns modelos propostos na Seção 2.2 para o modelagem de tempos de vida. A densidade do modelo, que foi motivada no contexto de confiabilidade é dada por

$$
f(t ; \sigma, \alpha)=\frac{2 \alpha t^{\alpha-1}}{\sigma^{\alpha}} \phi\left(\left(\frac{t}{\sigma}\right)^{\alpha}\right), \quad t>0,
$$

em que, $\sigma>0, \alpha>0$, e denotaremos por $T \sim H N G(\sigma, \alpha)$. Quando $\alpha=1$, temos o modelo halfnormal, enunciado na Seção 2.2. A taxa de risco e função de sobrevivência, respectivamente, para a distribuição half-normal generalizada são

$$
\begin{gathered}
h(t ; \sigma, \alpha)=\frac{\alpha t^{\alpha-1} \phi\left(\left(\frac{t}{\sigma}\right)^{\alpha}\right)}{\sigma^{\alpha}\left(1-\Phi\left(\left(\frac{t}{\sigma}\right)^{\alpha}\right)\right)}, \\
S(t ; \sigma, \alpha)=2\left(1-\Phi\left(\left(\frac{t}{\sigma}\right)^{\alpha}\right)\right) .
\end{gathered}
$$
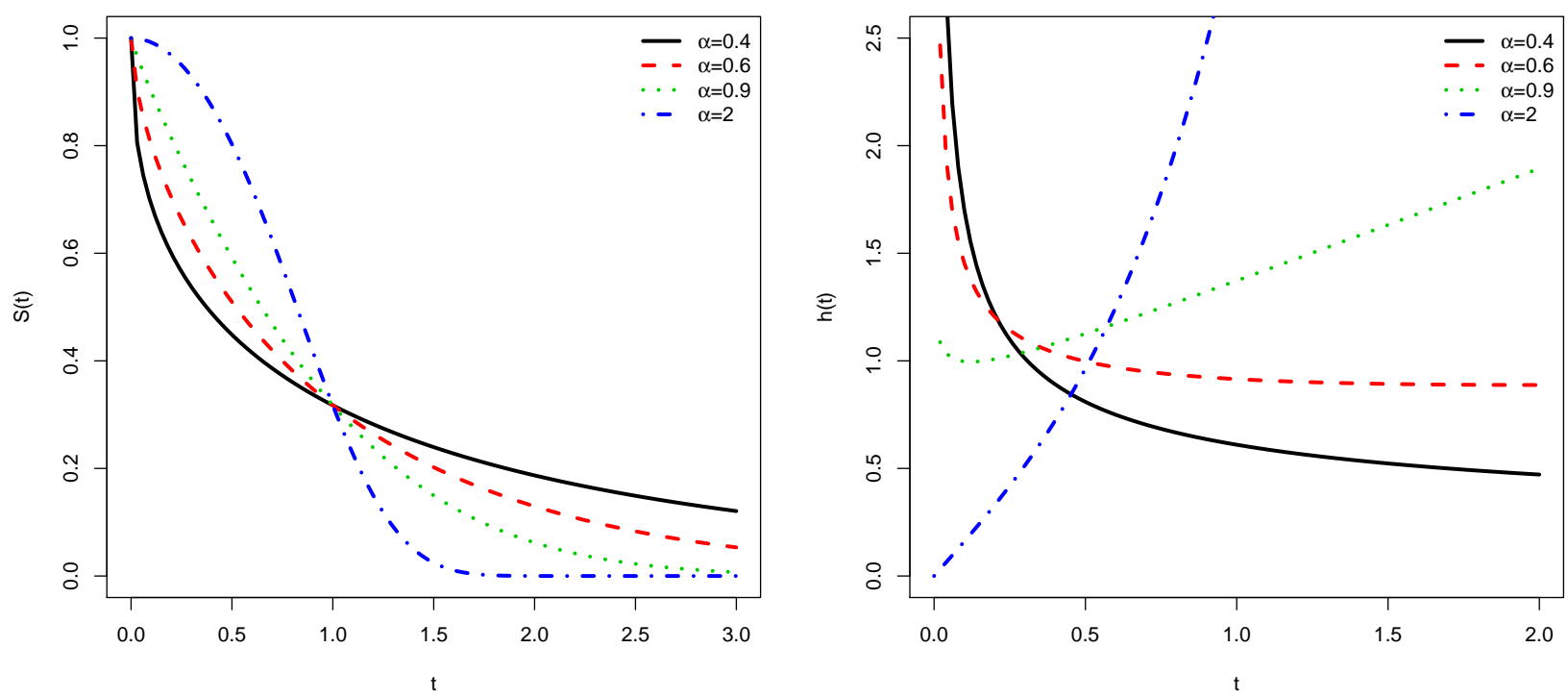

Figura 2.8: Função de sobrevivência (esquerda) e de risco (direita) para diferentes valores do parâmetro $\alpha$, do modelo HNG.

O parâmetro $\alpha$ afeta a forma desta nova distribuição. Em particular, dependendo dos valores do parâmetros, a função taxa de risco pode-se formar variedade de formas, tais como monótona crescente, monótona decrescente, e a forma banheira. As duas figuras seguintes demostram a forma da função de sobrevivência e a sua função de risco para diferentes valores de parâmetros (Figura $2.8)$.

Uma extensão mais recente deste modelo, foi proposto por Cordeiro et al. [2012], a qual utiliza a função de distribuição acumulada do modelo HNG, no generador Kumaraswamy-G introducida por Cordeiro e Castro [2015].

\subsubsection{Exponencial generalizada [Gupta e Kundu [1999]]}

A densidade do modelo é:

$$
f(t ; \alpha, \sigma)=\alpha \sigma \exp (-\sigma t)[1-\exp (-\sigma t)]^{\alpha-1}, \quad t>0,
$$


em que, $\sigma>0$, e $\alpha>0$, assim denotaremos por $T \sim E G(\sigma, \alpha)$. Quando $\alpha=1$, segue o modelo Exponencial, enunciado na Seção 2.2.

A taxa de risco e função de sobrevivência para o modelo são, respectivamente:

$$
h(t ; \sigma, \alpha)=\frac{\alpha \sigma \exp (-\sigma t)[1-\exp (-\sigma t)]^{\alpha-1}}{\left[1-(1-\exp \{-\sigma t\})^{\alpha}\right]}
$$

e

$$
S(t ; \sigma, \alpha)=1-(1-\exp \{-\sigma t\})^{\alpha} .
$$
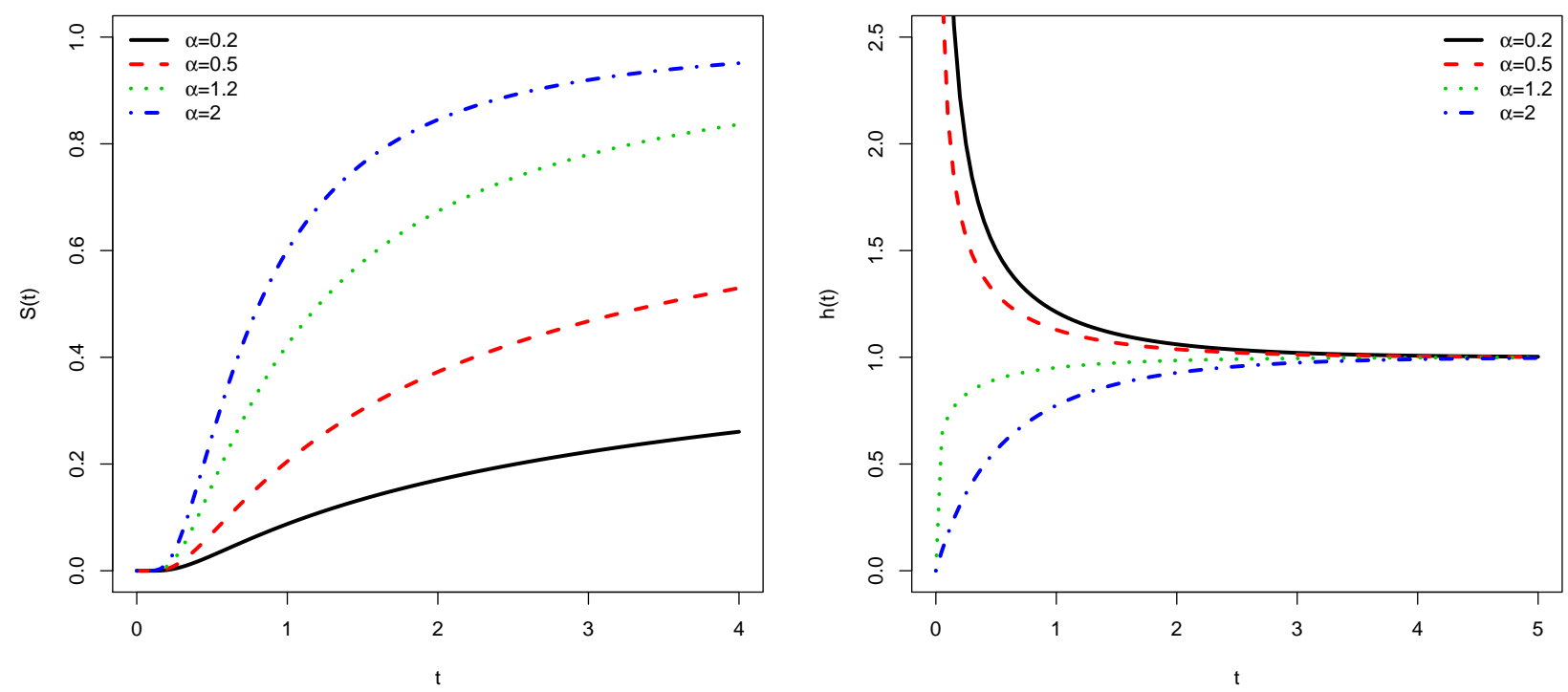

Figura 2.9: Função de sobrevivência (esquerda) e de risco (direita) para diferentes valores do parâmetro $\alpha$, do modelo $E G$.

\subsubsection{Uma extensão do modelo exponencial [Nadarajah e Haghighi [2011]]}

Consideremos uma extensão do modelo exponencial, onde a fdp do novo modelo é

$$
f(t ; \lambda, \alpha)=\alpha \lambda(1+\lambda t)^{\alpha-1} \exp \left\{1-(1+\lambda t)^{\alpha}\right\}, \quad t>0,
$$

em que, $\lambda>0$, e $\alpha>0$, que denotaremos por $T \sim N H E(\lambda, \alpha)$. Quando $\alpha=1$, temos como caso particular o modelo exponencial, enunciado na Seção 2.2 .

A taxa de risco e função de sobrevivência, respectivamente, para o modelo são:

$$
h(t ; \lambda, \alpha)=\alpha \lambda(1+\lambda t)^{\alpha-1}
$$

e

$$
S(t ; \lambda, \alpha)=\exp \left\{1-(1-(1+\lambda t))^{\alpha}\right\} .
$$

\subsection{Principais testes de bondade de ajuste}

Um teste de bondade de ajuste é um teste usado para determinar com um certo nível de significância se uma amostra observada $t_{1}, \ldots, t_{n}$, com tamanho $n$, foi obtida de uma certa distribuição. Quatro testes de bondade de ajuste serão considerados nesta seção, que são: Kolmogorov-Smirnov, teste da razão de verossimilhança (TRV), Cramér-von Mises e teste de Anderson-Darling. Estes, serão utilizados para verificar se os respectivos ajustes dos modelos aos conjuntos de dados que serão estudados são adequados. 

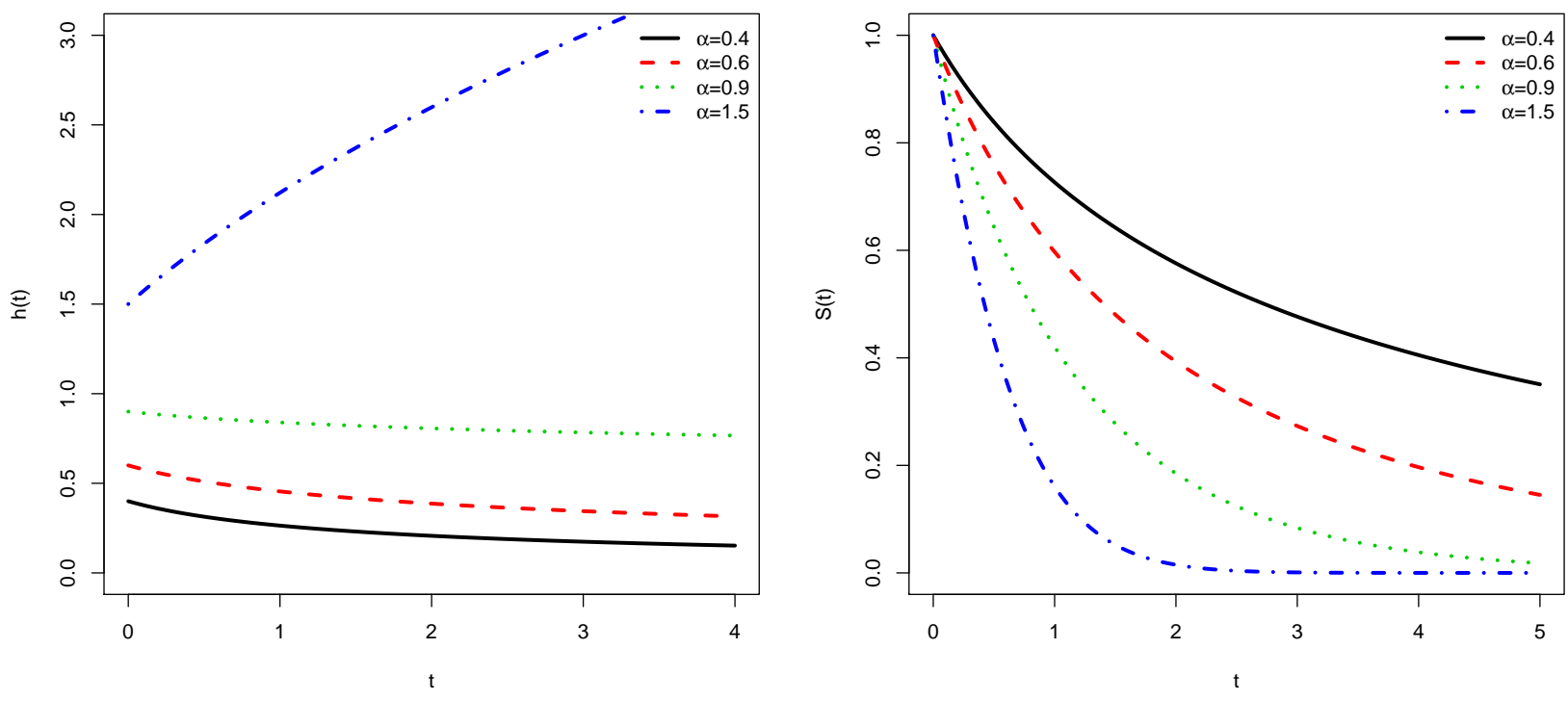

Figura 2.10: Função de sobrevivência (esquerda) e de risco (direita) para diferentes valores do parâmetro $\alpha$, do modelo ENH.

\subsubsection{Teste de Kolmogorov-Smirnov}

Sejam $t_{1}, \ldots, t_{n}$ uma amostra observada de uma variável aleatória com função de distribuição acumulada $F(t)$ e seja $k_{t}$ o número de observações $t_{i}<t$. A função de distribuição acumulada empírica é a função escada $\widehat{F(t)}=k_{t} / n$. A estatística de Kolmolgorov-Smirnov é definida como o máximo da diferença $|\widehat{F(t)}-F(t)|$, ou seja,

$$
K S=\max _{t}|\widehat{F(t)}-F(t)|,
$$

Kolmogorov [1933], Smirnov [1939].

\subsubsection{Teste da razão de verossimilhanças (TRV)}

O teste da razão de verossimilhanças poder ser usado para avaliar se um modelo se ajusta bem a um conjunto de dados. Quando usamos este teste para comparar dois modelos, eles devem ser encaixados, i.e, um dos modelos é caso particular do outro. O princípio para este teste é muito simples de seguir. Suponha que $T$ possua densidade denotada por $f(t ; \theta)$ com parâmetro desconhecido $\theta$. É de interesse testar a hipótese $H_{0}: \theta \in \Theta_{1}$ versus $H_{1}: \theta \in \Theta_{2}$, em que $\Theta_{1}$ e $\Theta_{2}$ são os espaços paramétricos para os modelos 1 e 2, respectivamente. A estatística da razão de verossimilhanças, denotado por TRV, pode ser escrita como

$$
T R V=-2 \log \left(\frac{L_{1}\left(\widehat{\theta_{1}}\right)}{L_{2}\left(\widehat{\theta_{2}}\right)}\right)
$$

em que $L 1$ e $L 2$ são as funções de verossimilhanças para os modelos 1 e 2 , respectivamente. O modelo 1 tem menos parâmetros do que o modelo 2. A estatística da razão de verossimilhanças (TRV) é distribuída (sob $H_{0}$ ) assintoticamente como a distribuição qui-quadrado com graus de liberdade igual a diferença entre o número de parâmetros livres sob os dois modelos. Rejeita-se o modelo 1 se $T R V>\chi_{\alpha, p_{1}-p_{2}}^{2}$ ao nivel de significância $\alpha$ em que $p_{1}$ e $p_{2}$ são os números de parâmetros livres para cada modelo. 


\subsubsection{Testes Anderson-Darling e Cramér-von Mises}

Os testes Cramer-von Mises e Anderson-Darling são testes comumente usados para testar se uma amostra aleatória $t_{1}, t_{2}, \ldots, t_{n}$ vem de uma distribuição especificada. $O$ primeiro foi proposto na década 1920-1930 por Cramer e von Mises, enquanto que o último foi introduzido por Anderson e Darling [1952]. Eles podem ser tratados como modificações do teste de KolmogorovSmirnov (KS), mas eles dão mais peso às caudas do que o teste de Kolmogorov-Smirnov. Esses testes dependem da distribuição específica para calcular os valores críticos. Este ponto pode ser considerado como uma vantagem resultando num teste mais sensível, ou uma desvantagem pois os valores críticos devem ser calculados para cada distribuição. A partir de agora, denotamos os testes de Cramér-von Mises e Anderson-Darling por CVM e AD, respectivamente. Sejam $F(t ; \theta)$ as fda's e $W^{*}$ e $A^{*}$ as estatísticas de teste Cramer-von Mises e Anderson-Darling, respectivamente. Descrevemo abaixo um algoritmo para implementar estes procedimentos.

- Calcular a função de distribuição $v_{i}=F\left(t_{i} ; \theta\right)$, em que os dados estão em ordem ascendente e, em seguida, calcular $y_{i}=\Phi^{-1}\left(v_{i}\right)$, em que $\Phi^{-1}$ é a função quantil da distribuição normal padrão $N(0,1)$.

- Calcular os valores padronizados de $y_{i}$, que podem ser obtidos por

$$
u_{i}=\left\{\left(y_{i}-\bar{y}\right) / s_{y}\right\},
$$

em que $\bar{y}$ é a média de $y_{i} ; i=1 ; 2 ; \ldots ; n$ e $s_{y}$ é o desvio padrão de $y_{i}$.

- Em seguida, as estatísticas de teste CVM e AD podem ser escritas como

$$
W^{2}=\sum_{i=1}^{n}\left(u_{i}-\frac{2 i-1}{2 n}\right)^{2}+\frac{1}{12 n},
$$

$\mathrm{e}$

$$
A^{2}=-n-\frac{1}{n} \sum_{i=1}^{n}\left\{(2 i-1) \log \left(u_{i}\right)+(2 n+1-2 i) \log \left(1-u_{i}\right)\right\} .
$$

- Para comparar os modelos usa-se aa versões modificadas das estatísticas, $W^{*}$ e $A^{*}$, que podem ser escritas como

$$
W^{*}=W^{2}\left(1+\frac{0.5}{n}\right),
$$

e

$$
A^{*}=A^{2}\left(1+\frac{0.75}{n}+\frac{2.25}{n^{2}}\right) .
$$

Para decidir se um modelo estatístico se ajusta bem a um conjunto de dados, comparamos os valores observados com os valores críticos de cada estatística. Se o valor observado é menor que o valor critico, rejeitamos a hipótese nula.

\subsection{Critérios de informação}

Sem dúvida, a construção de modelos estatísticos é fortemente dependente dos resultados de uma análise descritiva inicial dos dados observados. Às vezes, há uma grande diferença entre os resultados teóricos e procedimentos práticos. Na análise de um conjunto de dados, a seleção do melhor modelo para descrever os dados ainda é um problema considerável. Na seção anterior, apresentamos alguns testes de bondade de ajuste que são usados para comparar qual modelo é melhor para os dados, mas se esses modelos não são encaixados, então deve-se usar outros métodos. Um desses métodos é baseado nos critérios de informação. A seguir, apresentamos os critérios de informação mais utilizados, que denotamos por AIC, AICc, BIC e CAIC. 


\subsubsection{Critério de informação de Akaike}

O critério de informação de Akaike foi introduzido inicialmente em Akaike, H. [1973] e desenvolvido em Akaike, H. [1974]. Certamente é a ferramenta de seleção de modelos mais utilizada entre os pesquisadores. Para calcular o AIC, começamos com alguns modelos alternativos, que são considerados como modelos apropriados para certos conjuntos de dados. O critério de informação de Akaike pode ser escrito como

$$
A I C=-2 \log L(\widehat{\theta})+2 k
$$

em que $k$ é o número de parâmetros estimados para o modelo, $\log L(\widehat{\theta})$ é o logaritmo natural da função de verosimilhança e $\hat{\theta}$ o estimador de MV. Em seguida, pode-se dizer que o modelo adequado ao conjunto de dados é o modelo que apresenta o menor valor do AIC (em relação aos outros modelos alternativos).

Para amostras grandes, é possível provar que o AIC tende a aceitar o modelo correto com boa frequência (veja Shawky e Abu-Zinadah [2008]). No entanto, a tendência de escolher modelos com maior número de parâmetros parece ser um problema básico do critério, revelado em vários estudos (Findley [1985]), principalmente em amostras pequenas.

Uma modificação do AIC, o AICc foi proposto por Sugiura [1978], com vista a redução do viés apontado acima. Hurvich e Tsai [1989] mostraram que o critério modificado melhorou também a seleção do modelo correto em pequenas amostras.

Se o tamanho da amostra de dados $n$ é pequeno, ou se o modelo tem um grande número de parâmetros, $n / k<40$, então deve-se preferir o critério AICc

$$
A I C c=A I C+\frac{2 k(k+1)}{n-k-1} .
$$

AICc é adequado para modelos fortemente assimétricos.

\subsubsection{Critério de informação de Akaike consistente}

Bozdogan [1987] propõe uma extensão analítica do AIC sem prejudicar o principio básico do AIC. Ele é chamado critério de informação de Akaike consistente (CAIC). A expressão de CAIC é definida como

$$
C A I C=-2 \log L(\widehat{\theta})+k[\log (n)+1] .
$$

\subsubsection{Critério de informação Bayesiano}

Schwarz [1978] desenvolveu um novo critério, conhecido como Schwarz Information Criterion (SIC) ou Bayesian Information Criterion (BIC). A idéia principal do BIC vem de aproximar o fator de Bayes com a suposição de que os dados são independentes e identicamente distribuídos. Mesmo que este critério seja derivado dentro do contexto Bayesiano ele prefere modelos menos complexos, ao contrário do AIC, que prefere modelos mais complexos. Ele é definido como

$$
B I C=-2 \log L(\widehat{\theta})+k \log (n) .
$$

Como o AIC, o modelo adequado para um determinado conjunto de dados é o modelo com menor BIC, quando comparado com os outros. 


\section{Capítulo 3}

\section{Distribuição exponencial estendida}

Neste capitulo é proposta uma extensão do modelo exponencial baseado em mistura de distribuições com suporte positivo. Estudamos algumas propriedades, com especial ênfase nos momentos, função geradora de momentos e algumas caracteristicas relacionadas ao estudo de confiabilidade. Também discutimos a estimação dos parametros via máxima verossimilhança e o método dos momentos. Uma aplicação ao banco de dados reais é apresentada.

\subsection{Motivação}

O modelo exponencial, como foi detalhado no capítulo anterior, tem uma grande desvantagem para o modelagem de dados, pois sua taxa de risco é constante. É por isso que existem várias extensões da distribuição exponencial na literatura. Todas têm a finalidade de flexibilizar sua função de risco constante. Neste capítulo consideramos algumas extensões do modelo exponencial, como por exemplo, as propostas de Gupta e Kundu[1999, 2001], que introduziram a distribuição exponencial generalizada (EG) e a proposta de Nadarajah e Haghighi [2011], que introduziram uma extensão alternativa, que denominaremos ENH, apresentadas na Tabela 2.3 e nas expressões (2.23) e (2.26), respetivamente. Denotamos a variável aleatória como $T \sim E G(\alpha, \sigma)$ se tem distribuição exponencial generalizada e denotemos a variável aleatória como $T \sim E N H(\alpha, \lambda)$ se tem distribuição exponencial de Nadarajah e Haghighi, para mais detalhes destes modelos, vide a Seção 2.3

Ambas distribuições têm como caso particular o modelo exponencial quando $\alpha=1$, com parâmetro de escala $\sigma$ e $\lambda$, respetivamente.

O objetivo deste capítulo é apresentar uma nova extensão da distribuição exponencial que seja uma alternativa às distribuções EG e ENH.

\subsection{Funções de densidade e propriedades}

Proposição 3.2.1 Sejam $T \sim E E(\alpha, \beta), Y \sim \operatorname{Exponencial}(\alpha)$ e $Z \sim \operatorname{Gama}(2, \alpha)$. A função densidade do modelo EE é escrita como uma mistura entre $Y$ e $Z$, i.e.,

$$
T=p Y+(1-p) Z
$$

em que $p=\frac{\alpha}{\alpha+\beta}$.

Observação 1 A representação estocástica em (3.1) é útil no calculo de momentos de $T \sim E E(\alpha, \beta)$ e também na geração de valores da distribuição.

Propriedade 3.2.1 Uma variável aleatória $T$ tem distribuiçâo exponencial estendida (EE) com parâmetros $\alpha$ e $\beta$ se sua fdp é dada por

$$
f(t ; \alpha, \beta)=\frac{\alpha^{2}(1+\beta t) e^{-\alpha t}}{\alpha+\beta},
$$

em que $t>0, \alpha>0$ e $\beta \geq 0$. Denotemos a variável aleatória como $T \sim E E(\alpha, \beta)$.

Os seguintes são casos particulares do modelo EE:

1. Se $\beta=0$, obtemos o modelo exponencial. 
2. Se $\beta=1$, obtemos o modelo Lindley (sua função densidade pode ser vista na Tabela 2.2 ou na expressão (2.18).

\subsubsection{Principais propriedades}

Propriedade 3.2.2 A função de distribuição acumulada da variável aleatória $T$ é dada por

$$
F(t ; \alpha, \beta)=\frac{\alpha+\beta-(\beta+\alpha+\alpha \beta t) e^{-\alpha t}}{\alpha+\beta}, \quad t>0 .
$$
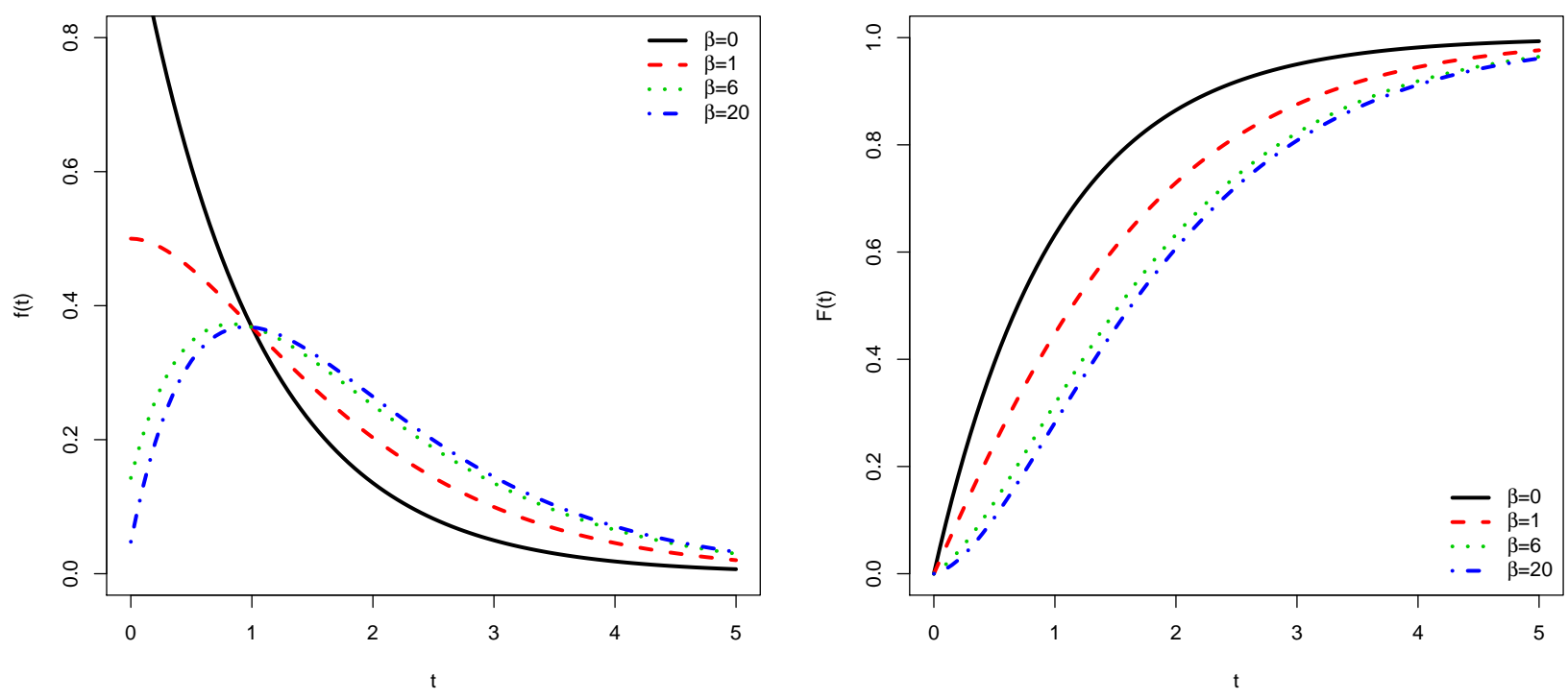

Figura 3.1: Gráficos da função de densidade(esquerda) e função de distribuição acumulada(direita) para $\alpha=1$, e $\beta=0,1,6,20$ do modelo $E E$.

A Figura 3.1 mostra a função densidade e a função de distribuição do modelo EE para valores de $\beta 0,1,6,20$. Neste gráfico, pode-se observar como o parâmetro $\beta$ afeta a forma da distribuição. Assim, para $\beta=0$, no caso exponencial, a curtose é menor que para $\beta=1$, o caso Lindley.

Definição 3.2.1 Sua função de sobrevivência é dada por

$$
S(t ; \alpha, \beta)=\frac{(\beta+\alpha+\alpha \beta t) e^{-\alpha t}}{\alpha+\beta}, \quad t>0 .
$$

Definição 3.2.2 A função de risco da variável aleatória $T \sim E E(\alpha, \beta)$ é

$$
h(t ; \alpha, \beta)=\frac{\alpha^{2}(1+\beta t)}{\beta+\alpha(1+\beta t)}, \quad t>0 .
$$

Algumas propriedades da função de risco, são:

i) Se $\beta=0$, então $h(t)=\alpha$, é a função de risco do modelo exponencial $\forall t>0$.

ii) $h(t)$ é monótona crescente com $h(0)=\frac{\alpha^{2}}{\alpha+\beta} \forall \beta \geq 0$.

iii) $h(t) \rightarrow \alpha$, quando $t \rightarrow \infty, \forall \beta \geq 0$.

iv) $h(t)$ é limitada, isto é, $\frac{\alpha^{2}}{\alpha+\beta} \leq h(t)<\alpha, \forall t>0$. 

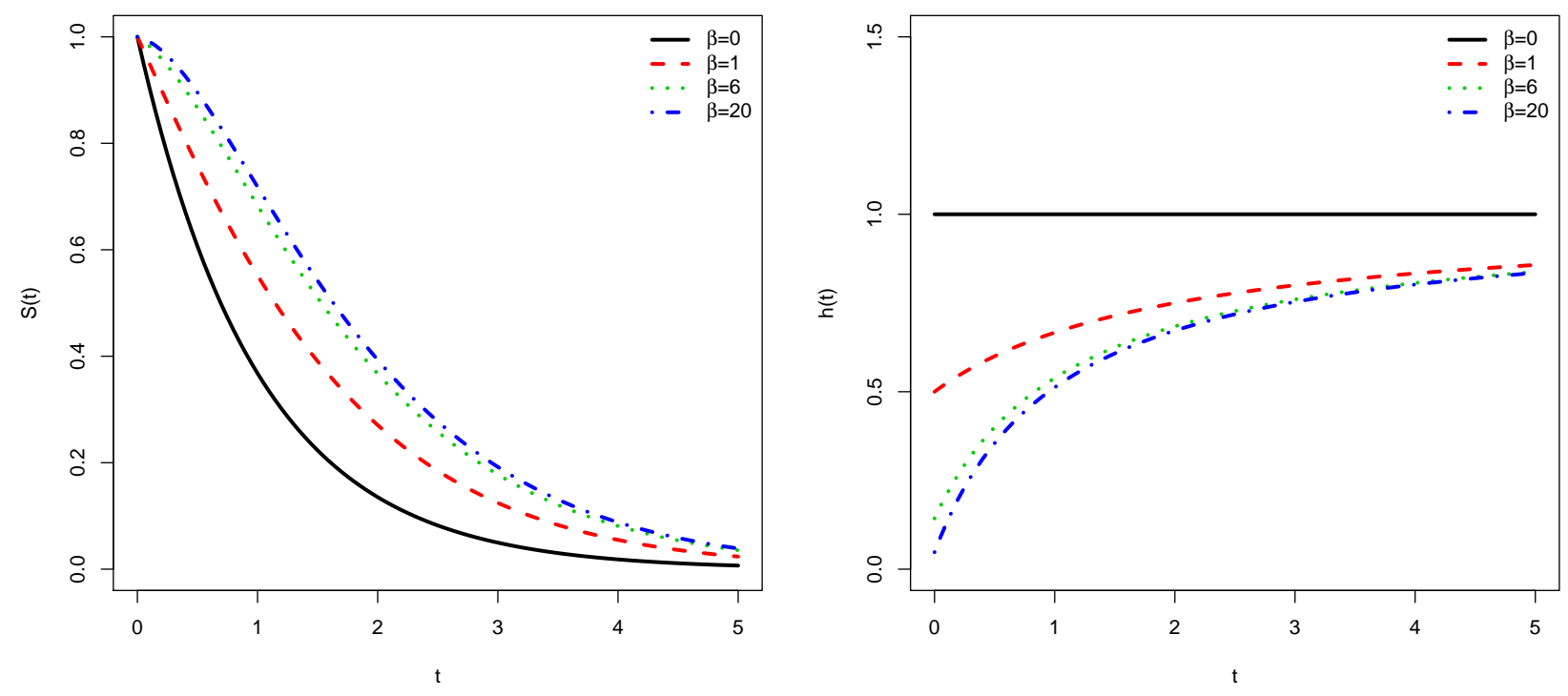

Figura 3.2: Gráficos da função de sobrevivência(esquerda) e função de risco(direita) para $\alpha=1$, e $\beta=$ 0,1,6,20 do modelo EE.

Propriedade 3.2.3 A função geradora de momentos dada por

$$
M_{X}(t)=\frac{\alpha^{2}(\alpha+\beta-t)}{(\alpha+\beta)(t-\alpha)^{2}}, \quad \text { parat } \geq 0
$$

Proposição 3.2.2 O r-ésimo momento da variável aleátoria $T \sim E E(\alpha, \beta)$ é dado por

$$
\mu_{r}=E\left(T^{r}\right)=\frac{r \Gamma(r)}{\alpha^{r}(1+\beta)}[\alpha+(1+r) \beta], \quad r=1,2, \ldots
$$

em que $\Gamma(\cdot)$ é a função gama, (vide expressão (2.9)).

\section{Prova}

Utilizando a representação estocástica em (3.1), pode-se expressar o momento $r$-ésimo como

$$
E\left(T^{r}\right)=\frac{\alpha}{\alpha+\beta} E\left(Y^{r}\right)+\frac{\beta}{\alpha+\beta} E\left(Z^{r}\right)=\frac{r \Gamma(r)}{\alpha^{r}(1+\beta)}[\alpha+(1+r) \beta], \quad r=1,2, \ldots
$$

concluindo a prova.

Corolário 3.2.1 Por conseguinte, os quatro primeiros momentos e a variância do modelo $E E(\alpha, \beta)$ são dados por

1. $\mu_{1}=E(T)=\frac{\alpha+2 \beta}{\alpha(\alpha+\beta)}$,

2. $\mu_{2}=E\left(T^{2}\right)=\frac{2(\alpha+3 \beta)}{\alpha^{2}(1+\beta)}$,

3. $\mu_{3}=E\left(T^{3}\right)=\frac{6(\alpha+4 \beta)}{\alpha^{3}(1+\beta)}$,

4. $\mu_{4}=E\left(T^{4}\right)=\frac{24(\alpha+5 \beta)}{\alpha^{4}(1+\beta)}$ 
5. $\operatorname{Var}(T)=\frac{\alpha^{3}+5 \alpha^{2} \beta+6 \alpha \beta^{2}+2 \beta^{3}}{\alpha^{5}+3 \alpha^{4} \beta+3 \alpha^{3} \beta^{2}+\alpha^{2} \beta^{3}}$.

Corolário 3.2.2 Os coeficientes de assimetria $\left(\sqrt{\beta_{1}}\right)$ e curtose $\left(\beta_{2}\right)$ são dados por

$$
\sqrt{\beta_{1}}=\frac{2(\alpha+2 \beta)^{3}-12 \beta^{2}(\alpha+\beta)}{\left(\alpha^{2}+4 \alpha \beta+2 \beta^{2}\right)^{3 / 2}}
$$

$e$

$$
\beta_{2}=\frac{3(\alpha+2 \beta)^{2}\left(3 \alpha^{2}+12 \alpha \beta+8 \beta^{2}\right)-72 \beta^{2}(\alpha+\beta)^{2}}{\left(\alpha^{2}+4 \alpha \beta+2 \beta^{2}\right)^{2}},
$$

respectivamente.

Observação 2 Notamos que se $\beta \rightarrow 0$, então $\sqrt{\beta_{1}} \rightarrow 2$ e $\beta_{2} \rightarrow 9$, que correspondem aos coeficientes de assimetria e curtose, respectivamente, do modelo exponencial. Em geral, os coeficientes de assimetria e curtose são tais que $\sqrt{2}<\sqrt{\beta_{1}} \leq 2$ e $6<\beta_{2} \leq 9$, respectivamente, como mostram as Figuras 3.3.

\subsubsection{Formas}

A primera derivada da função densidade da densidade 3.1 é dada por

$$
\frac{\partial f(t)}{\partial t}=\frac{\alpha^{2}}{\alpha+\beta} e^{-\alpha t}[\beta-\alpha(1+\beta t)]
$$

e a segunda derivada é

$$
\frac{\partial^{2} f(t)}{\partial t^{2}}=-\frac{\alpha^{2}}{\alpha+\beta} e^{-\alpha t}[1+\beta(1+t)]
$$

Então, temos que

i) Para $\alpha<\beta,\left.\frac{\partial^{2} f(t)}{\partial^{2}} f(t)\right|_{t=t_{0}}$ e $\left(\partial^{2} / \partial t_{0}^{2}\right) f\left(t_{0}\right)<0$ em que $t_{0}=\frac{\beta-\alpha}{\alpha \beta}$ é o único ponto crítico em que $f(t)$ é maximizada.

i) Para $\alpha>\beta,(\partial / \partial t) f(t) \leq 0$, i.e., $f(t)$ é decrescente em $t$.

Propriedade 3.2.4

$$
\operatorname{Moda}(T)= \begin{cases}\frac{\beta-\alpha}{\alpha \beta} & , \text { se } 0 \leq \alpha \leq \beta \\ 0 & , \text { caso contrário }\end{cases}
$$

Observação 3 Observe que a moda da distribuição exponencial é igual a 0 , enquanto que a moda da distribuição EE pode assumir outros valores como visto acima.

\subsection{Função quantílica}

Lembramos que a função quantílica de uma variável aleatória contínua $T$ é definida como a função

$$
Q(p)=F_{T}^{-1}(t), \quad 0<p<1 .
$$

Mais especificamente, a função quantílica da distribuição de EE pode ser expressa em forma fechada em termos da função de Lambert W Corless et al. [1996]. A função de Lambert W é definida como a solução da equação

$$
W(z) \exp (W(z))=z, \quad z \in \mathbb{C} .
$$

A função complexa de valores múltiplos $W$ tem dois ramos reais se $z$ é um número real tal que $z \geq-1 /$ e. O ramo real assumindo valores em $(-\infty,-1]$ é chamado de ramo negativo e denotado por $W_{-1}(z)$, em que $-1 / e \leq z<0$. 

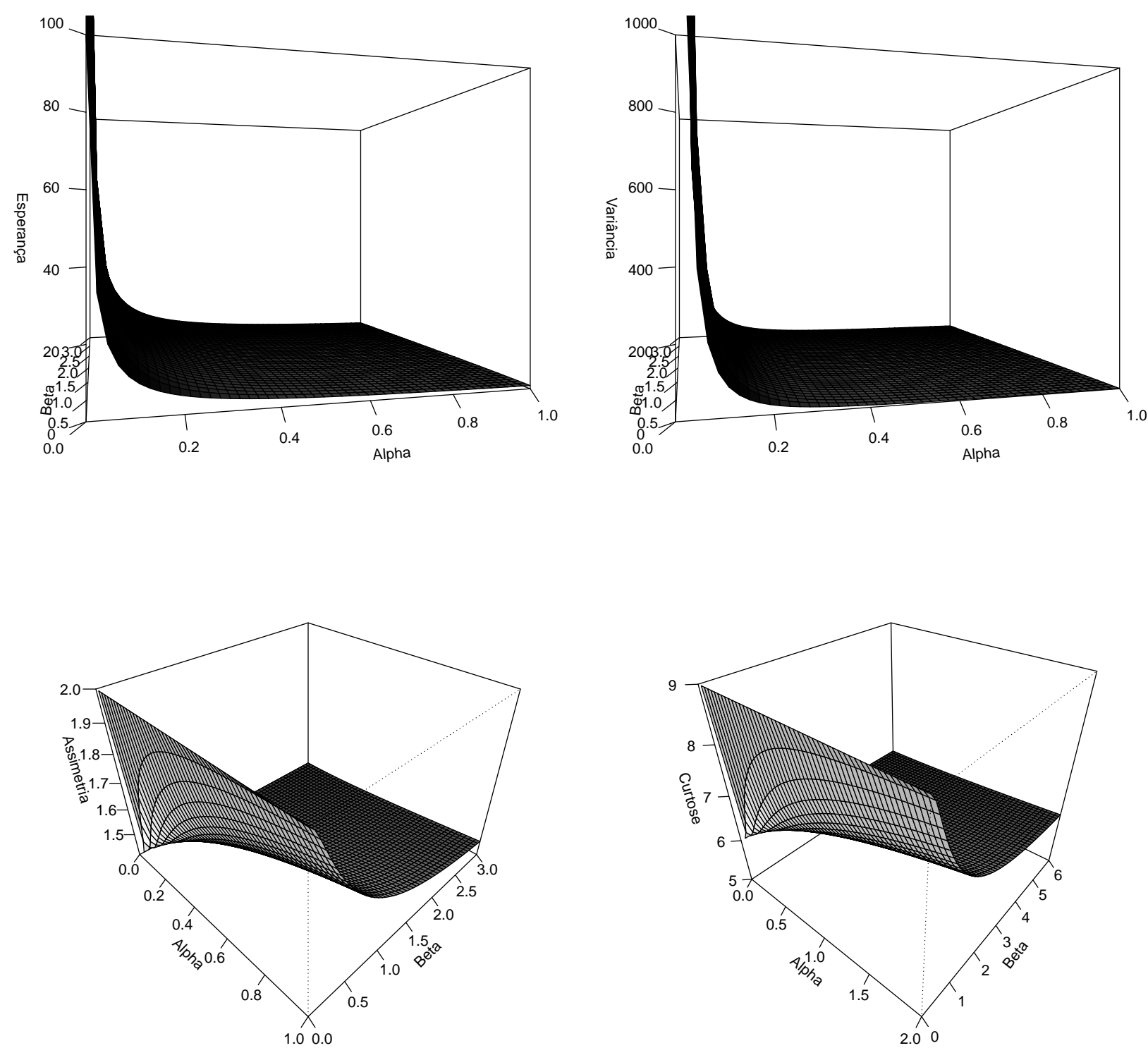

Figura 3.3: Algumas características da distribuição EE para diferentes conjuntos dos parâmetros. Painel superior esquerdo: média. Painel superior direito: variância. Painel inferior esquerdo: coeficiente de assimetria. Painel inferior direito: coeficiente de curtose.

Proposição 3.3.1 Seja $T$ uma variável aleatória com distribuição EE com parâmetros $\alpha>0$ e $\beta \geq 0$. A função quantílica de $T$ é dada por

$$
Q(p)=-\frac{1}{\beta}-\frac{1}{\alpha}-\frac{1}{\alpha} W_{-1}\left(\frac{(\alpha+\beta)(p-1)}{\exp \left(\frac{\alpha}{\beta}+1\right) \beta}\right), \quad 0<p<1,
$$

em que $W_{-1}$ que denota o ramo negativo da função de Lambert $W$.

\section{Prova}

Para qualquer $\alpha>0, \beta \geq 0$ e $p \in(0,1)$, temos que resolver a equação $F(t ; \alpha, \beta)=p$,com relação a 
$t$, em que $t>0$, isto é,

$$
\begin{aligned}
\frac{(\beta+\alpha+\alpha \beta t) e^{-\alpha t}}{\alpha+\beta} & =1-p, \\
(\beta+\alpha+\alpha \beta t) e^{-\alpha t} & =(1-p)(\alpha+\beta), \\
e\left(1+\frac{\alpha}{\beta}+\alpha t\right) e^{-\alpha t} & =\frac{(1-p)(\alpha+\beta)}{\beta} .
\end{aligned}
$$

Multiplicando por $\exp \left(-\frac{\alpha}{\beta}-1\right)$ ambos os lados, obtemos

$$
\left(-1-\frac{\alpha}{\beta}-\alpha t\right) \exp \left(-\alpha t-\frac{\alpha}{\beta}-1\right)=\frac{(\alpha+\beta)(p-1)}{\exp \left(\frac{\alpha}{\beta}+1\right) \beta}
$$

Da equação (3.5), em conjunto com (3.3), vemos que $-\left(1+\frac{\alpha}{\beta}+\alpha t\right)$ é a função de Lambert W do argumento real $\frac{(\alpha+\beta)(p-1)}{\exp \left(-\frac{\alpha}{\beta}-1\right) \beta}$. Então, temos

$$
W\left(\frac{(\alpha+\beta)(p-1)}{\exp \left\{\frac{\alpha}{\beta}+1\right\} \beta}\right)=-\left(1+\frac{\alpha}{\beta}+\alpha t\right)
$$

Além disso, para qualquer $\alpha>0, \beta \geq$ e $t>0$, é imediato que $-\left(1+\frac{\alpha}{\beta}+\alpha t\right)>1$ e pode ser verificado que $\frac{(\alpha+\beta)(p-1)}{\exp \left\{-\frac{\alpha}{\beta}-1\right\} \beta} \in(-1 / e, 0)$, desde que $p \in(0,1)$. Portanto, levando em consideração as propriedades do ramo negativo da função de Lambert $\mathrm{W}$ apresentada anteriormente, temos que

$$
W_{-1}\left(\frac{(\alpha+\beta)(p-1)}{\exp \left\{\frac{\alpha}{\beta}+1\right\} \beta}\right)=-\left(1+\frac{\alpha}{\beta}+\alpha t\right) .
$$

Assim, obtemos o resultado.

\subsection{Entropia de Shannon}

A entropia de uma variável aleatória $T$ contínua é uma medida de sua incerteza. A medida de entropia de Shannon é definida por

$$
J_{S}=-E\left(\log \left(f_{T}(t)\right)\right)
$$

Segue, depois de manipulações algébricas que a entropia de Shannon para o modelo EE é dada por

$$
J_{S}=-2 \log (\alpha)+\log (\alpha+\beta)+\alpha \log \left[\frac{\alpha+2 \beta}{\alpha(\alpha+\beta)}\right]-\log \left[1+\beta\left(\frac{\alpha+2 \beta}{\alpha(\alpha+\beta)}\right)\right] .
$$

Observação 4 Note-se que quando $\beta=1$, obtém-se, como caso particular a entropia de Shannon da distribuição Lindley, a qual é dada por

$$
\boldsymbol{J}_{S}=-2 \log (\alpha)+\log (\alpha+1)+\alpha \log \left[\frac{\alpha+2}{\alpha(\alpha+1)}\right]-\log \left[1+\left(\frac{\alpha+2}{\alpha(\alpha+1)}\right)\right],
$$

e quando $\beta=0$, obtém-se, como caso particular a entropia de Shannon da distribuição exponencial, que é dada por

$$
J_{S}=-\log (\alpha)(1+\alpha)
$$




\subsection{Inferência}

\subsubsection{Estimação pelo método dos momentos}

Os estimadores de momentos para os parâmetros $\alpha$ e $\beta$ são obtidos como solução das equações

$$
\frac{\alpha+2 \beta}{\alpha(\alpha+\beta)}=\bar{t}
$$

e

$$
\frac{2 \alpha+6 \beta}{\alpha^{2}(\alpha+\beta)}=\overline{t^{2}}
$$

em que $\bar{t}$ é a média amostral e $\overline{t^{2}}$ a média amostral dos cuadrados. Da primeira equação é obtido o estimador de momentos de $\beta(\widetilde{\beta})$ em função do estimador de momentos de $\alpha(\widetilde{\alpha})$,

$$
\widetilde{\beta}=\frac{\widetilde{\alpha}(1-\widetilde{\alpha} \bar{t})}{\widetilde{\alpha} \bar{t}-2}, \quad \widetilde{\alpha} \in\left(\frac{1}{\bar{t}}, \frac{2}{\bar{t}}\right) .
$$

Usando (3.7) e a segunda equação do sistema dado em (3.6) é obtido o estimador de momentos de $\alpha$ :

$$
\widetilde{\alpha}=\frac{2 \bar{t}+\sqrt{4 \bar{t}^{2}-2 \overline{t^{2}}}}{\overline{t^{2}}} .
$$

Logo, $\widetilde{\alpha}$ encontrado em (3.8) é substituido em (3.7) e se obtem $\widetilde{\beta}$. Os estimadores de momentos podem ser calculados analiticamente.

\subsubsection{Estimadores de máxima verossimilhança(EMV)}

\section{Equações de máxima verossimilhança}

Seja $t_{1}, t_{2}, \ldots, t_{n}$ observações de uma amostra aleatória de $T \sim E E(\alpha, \beta)$. A função de $\log$ verossimilhança fica dada por

$$
l(\alpha, \beta)=2 n \log (\alpha)-n \log (\alpha+\beta)-\alpha \sum_{i=1}^{n} t_{i}+\sum_{i=1}^{n} \log \left(1+\beta t_{i}\right) .
$$

Assim, as equações de verossimilhanças são dadas por

$$
\begin{aligned}
\frac{\partial l}{\partial \alpha} & =\frac{2 n}{\alpha}-\frac{n}{\alpha+\beta}-\sum_{i=1}^{n} t_{i}=0 \\
e & =-\frac{n}{\alpha+\beta}+\sum_{i=1}^{n} \frac{t_{i}}{1+\beta t_{i}}=0 .
\end{aligned}
$$

Da equação (3.9) são obtidos os estimadores de MV de $\beta$ e $\alpha$

$$
\widehat{\beta}=\frac{\widehat{\alpha}(1-\widehat{\alpha} \bar{t})}{\widehat{\alpha} \bar{t}-2}, \quad \widehat{\alpha} \in\left(\frac{1}{\bar{t}}, \frac{2}{\bar{t}}\right),
$$

em que o EMV de $\alpha$ é obtido resolvendo a equação

$$
\sum_{i=1}^{n} \frac{t_{i}}{1-(1-\bar{t} \widehat{\alpha})\left(\widehat{\alpha} t_{i}-1\right)}=\frac{n}{\widehat{\alpha}}
$$


em que $\widehat{\alpha}$ é a solução da equação (3.12), o qual deve ser substituido em (3.11) para obter $\widehat{\beta}$. Estes estimadores podem ser computados numericamente, usando, por exemplo, o lenguagem $\mathrm{R}$ R Core Team [2015] Desta forma são obtidos os estimadores de máxima verossimilança de $\alpha$ e $\beta$.

\subsection{Ilustração}

\subsubsection{Filamentos de Kevlar 49/epoxy a um de nível de estresse de $\mathbf{7 4 . 8 \%}$}

Nesta subseção apresentamos uma aplicação a um conjunto de dados reais relacionados com 76 observações que representam o tempo de resistência à ruptura de kevlar 49/epoxy que foram submetidos a pressão constante no nível de estresse $74.8 \%$ (373.9 Ksi) e uma temperatura de $110^{\circ} C$, até que todos tenham falhado, de modo que temos dados completos com tempos exatos de falha. Para estudos anteriores com este banco de dados, vide (Andrews e Herzberg [1985] e Barlow et al. [1984]). O conjunto de dados pode ser encontrado no Apêndice A.1. A Tabela 3.1 mostra algumas estatísticas descritiva dos dados, em que $\sqrt{b_{1}}$ e $b_{2}$ são os coeficientes de assimetria e curtose amostrais.

Tabela 3.1: Estatisticas descritivas para o conjunto de dados de vida de fratura por fatiga

\begin{tabular}{llllll}
\hline Conjunto de dados & $n$ & $\bar{t}$ & $s^{2}$ & $\sqrt{b_{1}}$ & $b_{2}$ \\
\hline \hline Kevlar & 76 & 1.959 & 2.477 & 2.019 & 8.600 \\
\hline
\end{tabular}

Usando os resultados da Seção 3.5.1, os estimadores de momentos obtidos resultaram $\widetilde{\alpha}=$ 0.889 e $\widetilde{\beta}=2.563$, que foram usados como estimativas iniciais para a abordagem de máxima verossimilhança. A Tabela 3.2 mostra as estimativas dos parâmetros para as distribuições EG,

Tabela 3.2: As estimativas dos parâmetros para os modelos EG, ENH, Lindley e EE para o conjunto de dados de vida de fratura por fatiga.

\begin{tabular}{lllll}
\hline Parâmetro & EG & ENH & Lindley & EE \\
\hline \hline$\alpha$ & 1.709 & 2.007 & - & 0.954 \\
$\beta$ & - & - & 0.795 & 6.366 \\
$\sigma$ & 0.703 & - & - & - \\
$\lambda$ & - & 0.195 & - & - \\
\hline \hline AIC & 248.49 & 253.48 & 249.35 & $\mathbf{2 4 7 . 3 0}$ \\
\hline
\end{tabular}

ENH, Lindley e EE usando máxima versossimilhança (EMV). Para esse conjunto de dados, o AIC mostra que o modelo que melhor ajusta é o modelo EE. A Figura 3.4 mostra o ajuste dos modelos.

\subsection{Observações finais}

Neste capítulo apresentamos um novo modelo para dados positivos. Mostra-se que o modelo pode ser representado como uma mistura de duas distribuições de suporte positivo. A distribuição exponencial com parâmetro de escala e a distribuição Lindley podem ser vistas como casos particulares do novo modelo. Mostra-se que a fda, função de risco e função de geradora de momentos têm forma fechada. Os estimadores de momentos são derivados e os estimadores de máxima verossimilhança podem ser calculados usando algoritmos do tipo Newton-Raphson. As estimativas de momentos podem ser usadas como valores iniciais para procedimentos numéricos de estimação por máxima verossimilhança. Os coeficientes de assimetria e de curtose são derivados. Ilustra-se o fato de que o modelo proposto tem maior flexibilidade em termos de coeficientes de assimetria e curtose. Na aplicação ao bancos de dados real verificamos que o modelo estudado é bastante útil e se comporta melhor em termos de ajuste do que outros modelos propostos recentemente na literatura, como os modelos EG e ENH. 

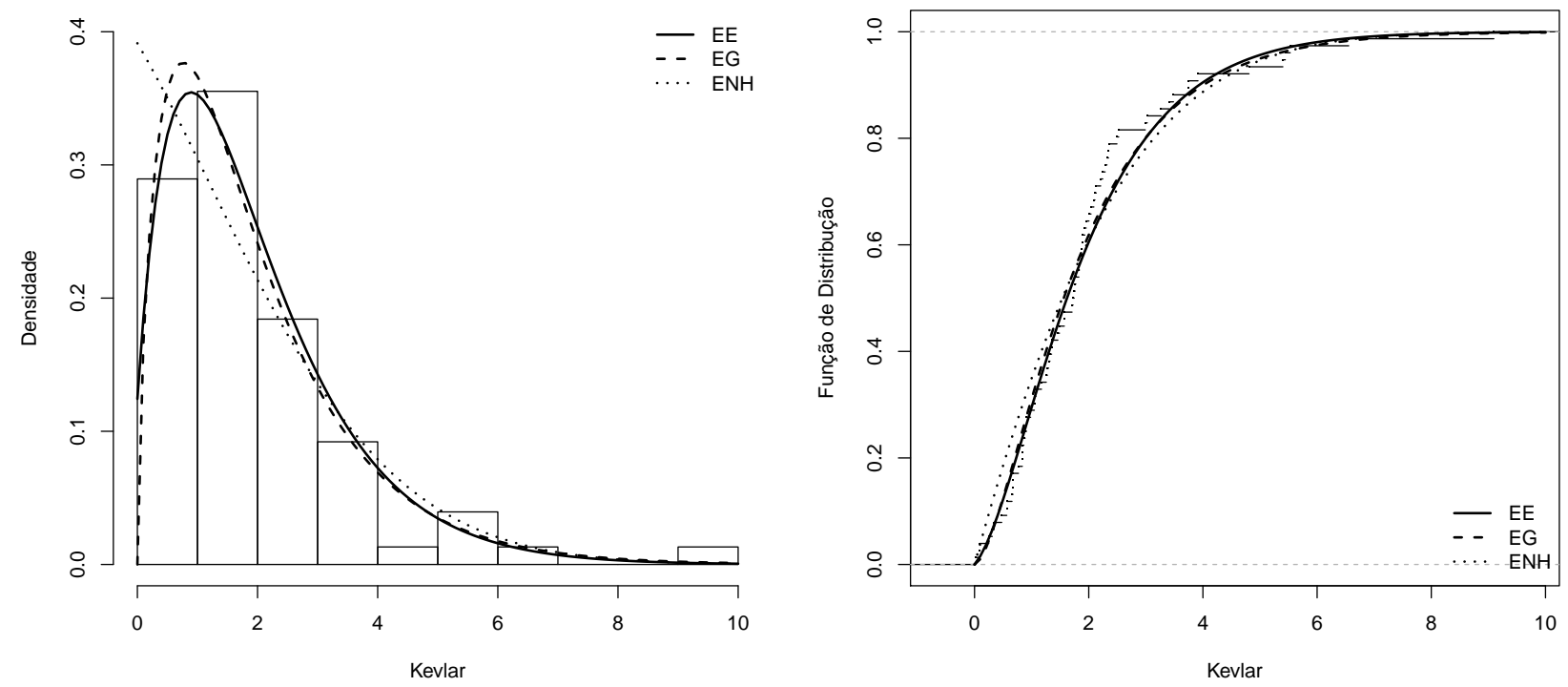

Figura 3.4: Modelos ajustados com a abordagem de máxima verossimilhança para o conjunto de dados de resistência à ruptura (esquerda) e fda empírica (direita) para o modelo EE. 


\section{Capítulo 4}

\section{O modelo Rayleigh-Lindley}

Neste capitulo é introduzida a distribuição Rayleigh-Lindley (RL), que é obtida compondo as distribuições Rayleigh e Lindley discreta. A distribuição resultante é um modelo de dois parâmetros competitivo com outros modelos parsimoniosos como as distribuiçôes gama e Weibull. Estudamos algumas propriedades deste novo modelo como os momentos e a vida média residual. A estimação dos parâmetros é abordada através dos métodos de máxima verossimilhança via algoritmo EM. O comportamento desses estimadores foi avaliado em um estudo de simulação. Finalmente, são descritas duas ilustrações a dados reais a fim de mostrar o desempenho do modelo versus outros dois modelos com dois parâmetros.

\subsection{Motivação}

As distribuições discretas tradicionais, tais como geométrica, Poisson, Weibull discreta (Nakagawa e Osake [1975])e gama discreta (Yang [1994]), entre outras, têm aplicabilidade limitada como modelos para confiabilidade, tempos de falha, biologia molecular e evolução. Neste contexto, o modelo Lindley discreto foi extensivamente estudado e é uma alternativa muito interessante para as distribuições discretas, considerando que tem apenas um parâmetro. Sua função de massa de probabilidade (fmp) é dada por

$$
P(M=m ; \theta)=\frac{p^{m-1}}{(1+\theta)}[\theta(1-2 p)+(1-p)(1+\theta(m-1))], \quad m=1,2, \ldots,
$$

em que $p=e^{-\theta}$ e $\theta>0$. Denotamos essa distribuição como $L D(\theta)$. A função de sobrevivência correspondente é

$$
S(M=m ; \theta)=\frac{1+\theta+\theta(m-1)}{1+\theta} p^{m-1}
$$

. A distribuição geométrica representa um caso limite quando $\theta \downarrow 0$. Para mais detalhes, vide Bakouch et al. [2014].

Por outro lado, a distribuição Rayleigh tem sido extensivamente utilizada para modelar dados em diversos campos de aplicação, incluindo confiabilidade e análise de sobrevivência. Sua função de densidade é dada por

$$
f_{X}(x ; \sigma)=\frac{x}{\sigma^{2}} e^{-\frac{x^{2}}{2 \sigma^{2}}}, \quad x \geq 0 .
$$

Denotamos como $X \sim R(\sigma)$. Suponha que, dado $M$, sejam $X_{1}, \ldots, X_{M}$ variáveis aleatórias seguindo a distribuição Rayleigh. Seja $T=\min \left(X_{1}, \ldots, X_{M}\right)$ tal que $M \sim D L(\theta)$. A distribuição marginal de $T$ será chamada como a distribuição Rayleigh-Lindley. Este procedimento de composição foi previamente realizado. Alguns exemplos são a exponencial geométrica (EGeo), exponencial Poisson (EP) e distribuições exponencial logarítmicas foram introduzidas por Adamidis e Loukas [1998], Kus [2007], Tahmasbi e Rezaei [2008], respectivamente, e Morais e Barreto-Souza [2011], que introduzem a familia série de potência Weibull (SPW). Por outro lado, extensões da distribuição de Rayleigh foram discutidas em Kundu e Raqab [2005], Manesh e Khaledi [2008], Cordeiro et al. [2013] e Iriarte et al. [2015].

A vantagem do modelo é a sua interpretação. Em muitos problemas aplicados a ocorrência de falhas pode ser causada por uma série de fatores desconhecidos que podem atuar em conjunto ou indivi- 
dualmente. A variável $X_{i}$ representa o tempo que o elemento $i$ leva a ocorrência dado o evento de interesse, que consideramos segue a distribuição de Rayleigh e que é suficiente a ocorrência de um desses elementos para a ocorrência do evento de interesse.

O principal objetivo deste capítulo é estudar a distribuição RL, que pode ser usada como uma alternativa para os modelos Weibull, gama e exponencial, entre outros. Discutimos algumas propriedades para esta nova distribuição como seus momentos, e alguns procedimentos de estimação.

\subsection{O modelo}

\subsubsection{Funções de densidade e sobrevivência}

Proposição 4.2.1 Seja $T \sim R L(\theta, \sigma)$. Então, a função de sobrevivência de $T$ é dada por

$$
S_{T}(t ; \sigma, \theta)=\frac{S_{X}(t ; \sigma)\left\{(1+\theta) p^{2} S_{X}(t ; \sigma)-p\left(2 \theta+1+S_{X}(t ; \sigma)\right)+1+\theta\right\}}{(1+\theta)\left[1-p S_{X}(t ; \sigma)\right]^{2}},
$$

em que $p=\exp (-\theta), S_{X}(x ; \sigma)=\exp \left(-\frac{x^{2}}{2 \sigma^{2}}\right)$ é a função de sobrevivência da distribuição Rayleigh e $\sigma, \theta>0, t>0$. Vamos denotar $T \sim R L(\theta, \sigma)$ como a distribuição Rayleigh-Lindley.

Prova Usando a representação hierárquica, temos que

$$
\begin{aligned}
X_{1}, \ldots, X_{M} \mid M=m ; \sigma & \sim R(\sigma) e \\
M ; \theta & \sim D L(\theta) .
\end{aligned}
$$

Pode-se mostrar que $T \mid M=m \sim R(\sigma / \sqrt{m})$, em que $T=\min \left(X_{1}, \ldots, X_{M}\right)$. Por conseguinte, a função de sobrevivência marginal de $T$ é dada por

$$
\begin{aligned}
S_{T}(t ; \sigma, \theta) & =\sum_{m=1}^{\infty} S_{T}(t \mid M=m ; \sigma) P(M=m ; \theta) \\
& =\frac{1}{(1+\theta)} \sum_{m=1}^{\infty} \exp \left(-\frac{m t^{2}}{2 \sigma^{2}}\right) p^{m-1}[\theta(1-2 p)+(1-p)(1+\theta(m-1))] .
\end{aligned}
$$

Depois de algumas manipulações algébricas, o resultado é obtido.

Corolário 4.2.1 Se $T \sim R L(\theta, \sigma)$, então a função de densidade de probabilidade de $T$ é dada por

$$
f_{T}(t ; \sigma, \theta)=\frac{f_{X}(t ; \sigma)\left\{p^{2} S_{X}(t ; \sigma)-p\left[(1-\theta) S_{X}(t ; \sigma)+2 \theta+1\right]+1+\theta\right\}}{(1+\theta)\left[1-p S_{X}(t ; \sigma)\right]^{3}}
$$

e sua respectiva função taxa de risco é dada por

$$
h_{T}(t ; \sigma, \theta)=\frac{t\left[1-p S_{X}(t ; \sigma)\right]\left(p^{2} S_{X}(t ; \sigma)-p\left[(1-\theta) S_{X}(t ; \sigma)+2 \theta+1\right]+1+\theta\right)}{\sigma^{2}\left((1+\theta) p^{2} S_{X}(t ; \sigma)-p\left(2 \theta+1+S_{X}(t ; \sigma)\right)+1+\theta\right)},
$$

em que $p=\exp (-\theta), f_{X}(x ; \sigma)=\frac{x}{\sigma^{2}} e^{-\frac{x^{2}}{2 \sigma^{2}}}$ é a função de densidade da distribuição Rayleigh, $S_{X}(x ; \sigma)=\exp \left(-\frac{x^{2}}{2 \sigma^{2}}\right)$ é a função de sobrevivência da distribuição Rayleigh, e $\sigma, \theta>0, t>0$.

Observação 5 Nota-se que as funções de densidade, de sobrevivência e de distribuição acumulada 
podem ser escritas como

$$
\begin{aligned}
f_{T}(t ; \sigma, \theta) & =\frac{f_{X}(t ; \sigma)}{\eta(\theta)\left\{1-p S_{X}(t ; \sigma)\right\}^{3}}\left\{\gamma(\theta) S_{X}(t ; \sigma)+\beta(\theta)\right\} \\
S_{T}(t ; \sigma, \theta) & =\frac{S_{X}(t ; \sigma)}{\eta(\theta)\left\{1-p S_{X}(t ; \sigma)\right\}^{2}}\left\{\alpha(\theta) S_{X}(t ; \sigma)+\beta(\theta)\right\} \\
e & =\frac{1}{\eta(\theta)\left\{1-p S_{X}(t ; \sigma)\right\}^{2}}\left\{\delta(\theta) S_{X}^{2}(t ; \sigma)+\varepsilon(\theta) S_{X}(t ; \sigma)+\eta(\theta)\right\},
\end{aligned}
$$

$$
\begin{aligned}
\operatorname{com} & & & \\
\alpha(\theta) & =p^{2}(1+\theta)-p, & & \beta(\theta)=-(2 \theta+1) p+1+\theta, \\
\gamma(\theta) & =p(p+\theta-1), & & \delta(\theta)=p, \\
\varepsilon(\theta) & =-(1+\theta+p) e & & \eta(\theta)=1+\theta .
\end{aligned}
$$

Usamos esta notação a fim de estudar algumas propriedades desta distribuição.

\subsection{Principais propriedades}

Nesta seção algumas propriedades básicas da distribuição Rayleigh-Lindley são consideradas. Seja $T \sim R L(\sigma, \theta)$. Então,

1. $\lim _{\theta \rightarrow \infty} S_{T}(t ; \sigma, \theta)=e^{-\frac{t^{2}}{2 \sigma^{2}}}, \quad \forall t \geq 0$,

2. $\lim _{\theta \rightarrow 0^{+}} S_{T}(t ; \sigma, \theta)=0, \quad \forall t>0 \quad e$

3. $\lim _{\theta \rightarrow 0^{+}} S_{T}(0 ; \sigma, \theta)=1$.

Observação 6 A Propriedade 1 revela que, quando $\theta \rightarrow \infty$ a distribuição $R L$ converge para a distribuição de Rayleigh usual. As Propriedades 2 e 3 indicam que quando $\theta \rightarrow 0^{+}$, a distribuição $R L$ converge para uma variável aleatória degenerada em zero. A Figura 4.1 ilustra algumas das formas que a distribuição Rayleigh-Lindley pode assumir para diferentes valores de seus parâmetros.
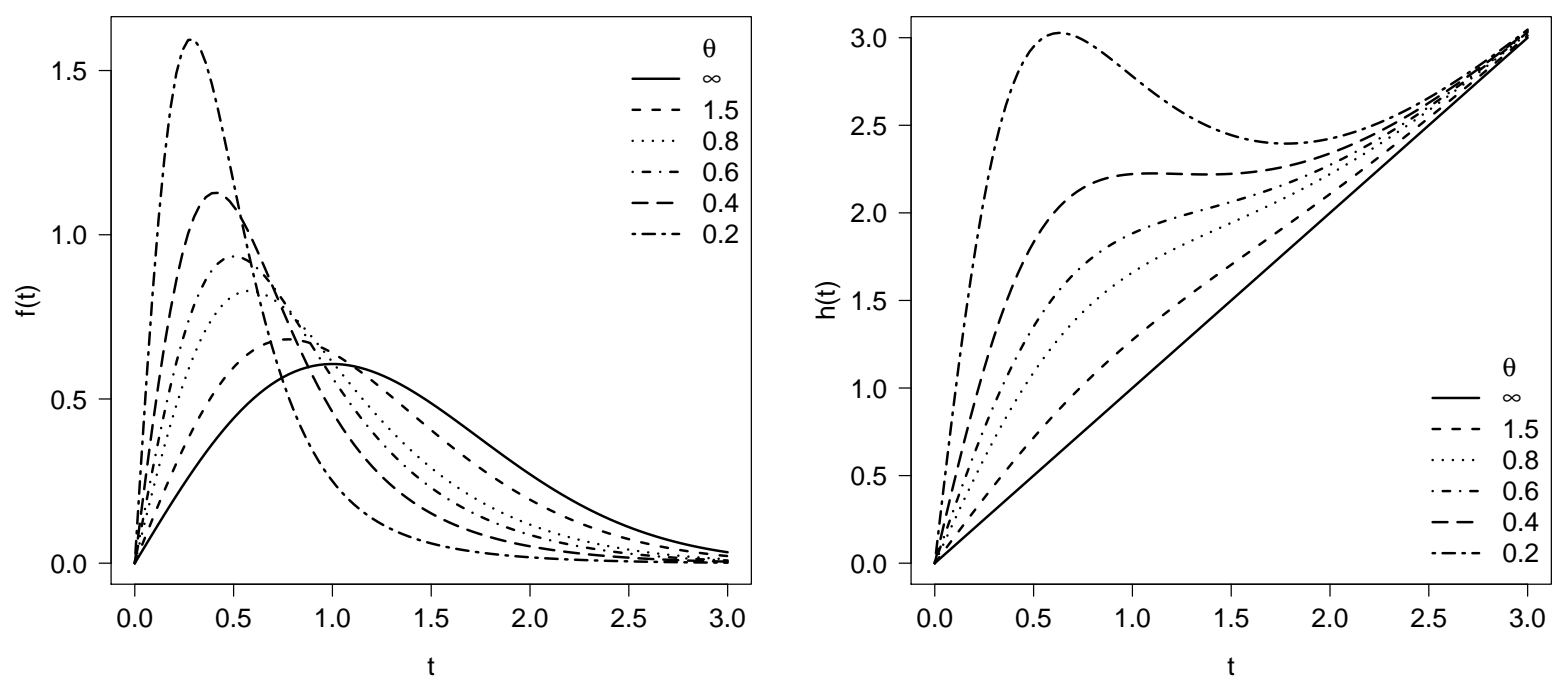

Figura 4.1: Gráfico das funções de densidade e taxa de risco para a distribuição RL. 


\subsubsection{Momentos e medidas complementares}

Os momentos da distribuição RL podem ser expressos em forma fechada em termos da função polilogaritmo. Lembramos que a função polilogaritmo de ordem $\lambda$ e argumento $z$ é definida pela série de Dirichlet

$$
\operatorname{Li}_{\lambda}(z):=\sum_{i=1}^{\infty} \frac{z^{i}}{i^{\lambda}}
$$

que é absolutamente convergente para todo complexo $\lambda$ e $z$ no interior do círculo unitário no plano- $z$ complexo (vide Apostol [2010] para mais detalhes).

Proposição 4.3.1 Se $T \sim R L(\sigma, \theta)$, então

$$
E\left(T^{r}\right)=\left(2 \sigma^{2}\right)^{r / 2} \frac{\Gamma\left(\frac{r}{2}+1\right)}{(1+\theta) p}\left[\theta(1-p) \operatorname{Li}_{\frac{r}{2}-1}(p)+(1-p-\theta p) \operatorname{Li}_{\frac{r}{2}}(p)\right], \quad r=1,2, \ldots
$$

Prova Para qualquer $r=1,2, \ldots$, o $r$-ésimo momento de $T$ pode ser calculado como segue:

$$
E\left[T^{r}\right]=\int_{0}^{\infty} t^{r} f(t ; \sigma, \theta) d t
$$

Ao fazer a mudança de variável $u=S_{X}(t ; \sigma)$ obtemos

$$
E\left[T^{r}\right]=\frac{\left(2 \sigma^{2}\right)^{r / 2}}{1+\theta} I_{r}(\theta)
$$

com

$$
I_{r}(\theta)=\int_{0}^{1}\{\log (1 / u)\}^{r / 2} \frac{\gamma(\theta) u+\beta(\theta)}{(1-p u)^{3}} d u .
$$

Do Corolário 2.6 em Jodrá e Jiménez-Gamero [2014], temos

$$
I_{r}(\theta)=\gamma(\theta) \frac{\Gamma\left(\frac{r}{2}+1\right)}{2 p^{2}}\left\{\operatorname{Li}_{\frac{r}{2}-1}(p)-\operatorname{Li}_{\frac{r}{2}}(p)\right\}+\beta(\theta) \frac{\Gamma\left(\frac{r}{2}+1\right)}{2 p}\left\{\operatorname{Li}_{\frac{r}{2}-1}(p)+\operatorname{Li}_{\frac{r}{2}}(p)\right\}
$$

e, portanto, o resultado.

A importância prática do resultado da Proposição 4.3.1 é que ele nos fornece uma maneira fácil de calcular os momentos de $T$ porque a função polilogaritmo está implementada nos sistemas de álgebra computacional como Maple e Mathematica, bem como na linguagem $\mathrm{R}$ [R Core Team, 2015] (vide, por exemplo, a função polylog dos pacotes pracma e copula).

Corolário 4.3.1 Se $T \sim R L(\sigma, \theta)$, então

$$
\begin{aligned}
E(T)= & \sqrt{\frac{\pi}{2}} \frac{\sigma}{p(1+\theta)}\left[\theta(1-p) \operatorname{Li}_{-\frac{1}{2}}(p)+(1-p-\theta p) \operatorname{Li}_{\frac{1}{2}}(p)\right], \\
\operatorname{Var}(T)= & \frac{2 \sigma^{2}}{(1+\theta) p^{2}}[\theta p-(1-p-\theta p) \log (1-p)] \\
& -\frac{\pi \sigma^{2}}{(1+\theta)^{2} p^{2}}\left[\theta(1-p) \operatorname{Li}_{-\frac{1}{2}}(p)+(1-p-\theta p) \operatorname{Li}_{\frac{1}{2}}(p)\right]^{2} .
\end{aligned}
$$

Corolário 4.3.2 Se $T \sim R L(\sigma, \theta)$, os coeficientes de assimetria $\left(\sqrt{\beta_{1}}\right)$ e curtose $\left(\beta_{2}\right)$ são, respectivamente,

$e$

$$
\sqrt{\beta_{1}}=\frac{\sqrt{\pi}\left[\frac{3}{4} \kappa_{3}(\theta)-\frac{3}{p \eta(\theta)} \kappa_{1}(\theta) \kappa_{2}(\theta)+\frac{2 \pi^{3}}{p^{2} \eta(\theta)^{2}} \kappa_{1}^{2}(\theta)\right]}{\eta(\theta) p\left[\frac{1}{p \eta(\theta)} \kappa_{2}(\theta)-\frac{\pi}{4 p^{2} \eta(\theta)^{2}} \kappa_{1}^{2}(\theta)\right]^{3 / 2}}
$$

$$
\beta_{2}=\frac{\frac{2}{p \eta(\theta)} \kappa_{4}(\theta)-\frac{3 \pi}{2 p^{2} \eta(\theta)^{2}} \kappa_{1}(\theta) \kappa_{3}(\theta)+\frac{6 \pi}{4 \eta(\theta)^{3} p^{3}} \kappa_{1}^{2}(\theta) \kappa_{2}(\theta)-\frac{3 \pi^{2}}{16 \eta(\theta)^{4} p^{4}} \kappa_{4}(\theta)}{\left(\frac{1}{\eta(\theta) p} \kappa_{2}(\theta)-\frac{1}{p^{2} \eta(\theta)} \kappa_{1}^{2}(\theta)\right)^{2}}
$$


em que $\kappa_{r}(\theta)=-\frac{\alpha(\theta)}{p} \operatorname{Li}_{\frac{r}{2}}(p)+\theta(1-p) \operatorname{Li}_{\frac{r}{2}-1}(p), \quad r=1,2,3,4$.

Observação 7 Quando $\theta \rightarrow \infty$, os coeficientes $\sqrt{\beta_{1}}$ e $\beta_{2}$, respectivamente, tendem a

$$
\sqrt{\beta_{1}}=\frac{2 \sqrt{\pi}(\pi-3)}{(4-\pi)^{3 / 2}} \quad \text { e } \quad \beta_{2}=\frac{32-3 \pi^{2}}{(4-\pi)^{2}}
$$

que são os coeficientes de assimetria e curtose da distribuição Rayleigh. A Figura 4.2 mostra os coeficientes de assimetria e curtose da distribuição RL.
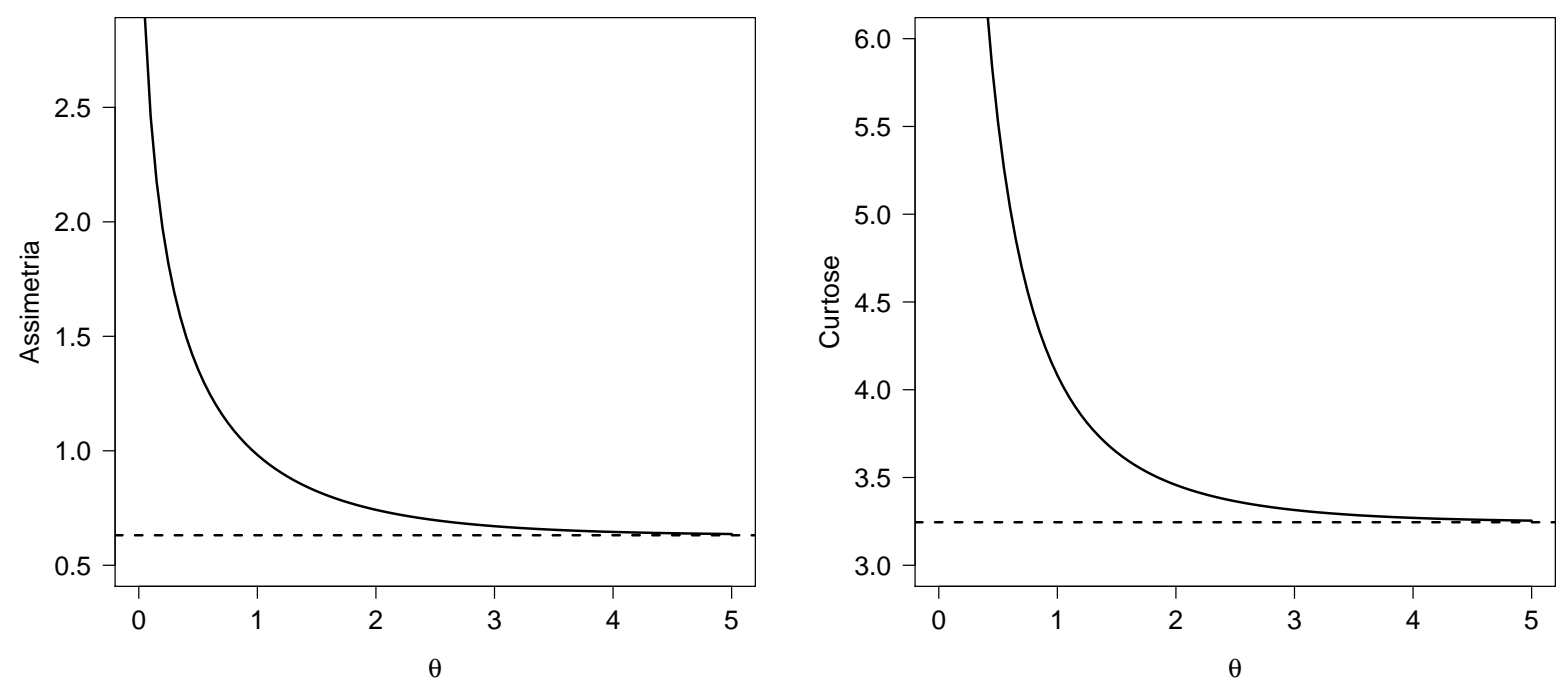

Figura 4.2: Gráfico dos coeficientes de assimetria e curtose para o modelo $R L$.

\subsubsection{Vida média residual}

A vida média residual de uma variável aleatória positiva $T$ com função de sobrevivência $S(t)$ é definida como

$$
e(t)=E(T-t \mid T>t)=\frac{\int_{t}^{\infty} S(y) d y}{S(t)}, \quad t>0 .
$$

Proposição 4.3.2 Para $T \sim R L(\sigma, \theta)$, a vida média residual é dada por

$$
e(t)=\frac{(2 \pi)^{1 / 2} \sigma\left(1-p S_{X}(t ; \sigma)\right)^{2} \sum_{m=1}^{\infty} m^{-1 / 2} p^{m-1}[\kappa(\theta)+\theta(1-p) m] \Phi\left(-\frac{m t}{\sigma^{2}}\right)}{S_{X}(t ; \sigma)\left[\alpha(\theta) S_{X}(t ; \sigma)+\beta(\theta)\right]}, \quad t>0,
$$

em que $\kappa(\theta)=1-p-\theta p$.

Note que a série do numerador é convergente, pois

$$
\sum_{m=1}^{\infty} m^{-3 / 2} p^{m-1} \Phi\left(-\frac{m t}{\sigma^{2}}\right) \leq \sum_{m=1}^{\infty} m^{-1 / 2} p^{m-1} \Phi\left(-\frac{m t}{\sigma^{2}}\right) \leq \sum_{m=1}^{\infty} p^{m-1}<\infty .
$$

Portanto, para fins numéricos a expressão em (4.1) pode ser aproximada por uma sequência finita de valores. 


\subsection{Estimação}

\subsubsection{Método dos momentos}

Sejam $t_{1}, \ldots, t_{n}$ observações de uma amostra aleatória a partir da distribuição $R L(\sigma, \theta)$. Os estimadores de momentos são a solução para as equações

$$
E(T)=\bar{t} \quad \text { e } \quad \operatorname{Var}(T)=\overline{t^{2}},
$$

em que $\overline{t^{j}}=n^{-1} \sum_{i=1}^{n} t_{i}^{j}, j=1,2$. Para $\sigma$, esta solução é dada por

$$
\widetilde{\sigma}_{\text {mom }}=\sqrt{\frac{2}{\pi}}(1+\theta) p \bar{t}^{2}\left[\{\theta(1-p)\} \operatorname{Li}_{\frac{1}{2}}(p)+(1-p-\theta p) \operatorname{Li}_{\frac{-1}{2}}(p)\right]^{-1},
$$

enquanto que $\widetilde{\theta}_{\text {mom }}$ é a solução para a seguinte equação

$$
g_{1}(\theta)=B_{t}
$$

$\operatorname{com} B_{t}=\overline{t^{2}} / \bar{t}^{2} \mathrm{e}$

$$
g_{1}(\theta)=\frac{4(1+\theta) p[\theta p-(1-p-\theta p) \log (1-p)]}{\pi\left[\theta(1-p) \operatorname{Li}_{\frac{1}{2}}(p)+(1-p-\theta p) \operatorname{Li}_{\frac{-1}{2}}(p)\right]^{2}} .
$$

\subsubsection{Algoritmo EM para dados censurados}

Nesta subseção discutiremos a estimativa dos parâmetros para uma amostra censurada do modelo RL. Sejam $Z_{1}, \ldots, Z_{n}$ e $C_{1}, \ldots, C_{n}$ os tempos de falhas e de censura à direita para cada observação, respectivamente. Sejam $T_{i}=\min \left(Z_{i}, C_{i}\right)$ e $\delta_{i}=I\left(Z_{i} \leq C_{i}\right)$ os tempos observados e os indicadores de falha, $i=1, \ldots, n$, em que $I(\cdot)$ é a função indicadora. Adicionando a suposição de que $T_{i}$ e $C_{i}$ são independentes e que a censura é não informativa, a função de log-verossimilhança para $\boldsymbol{\psi}=(\theta, \sigma)$ é dada por

$$
\begin{aligned}
\ell(\boldsymbol{\psi})= & \sum_{i=1}^{n}\left[\log S_{T}\left(t_{i} ; \boldsymbol{\psi}\right)+\delta_{i} \log h_{T}\left(t_{i} ; \boldsymbol{\psi}\right)\right] \\
= & -n \log (1+\theta)+\sum_{i=1}^{n}\left\{-\left(2+\delta_{i}\right) \log \left(1-p S_{X}\left(t_{i} ; \sigma\right)\right)-\frac{t_{i}^{2}}{2 \sigma^{2}}+\delta_{i}\left(\log t_{i}-2 \log \sigma\right)\right. \\
& \left.+\delta_{i} \log \left(\gamma(\theta) S_{X}\left(t_{i} ; \sigma\right)+\beta(\theta)\right)+\left(1-\delta_{i}\right) \log \left(\alpha(\theta) S_{X}\left(t_{i} ; \sigma\right)+\beta(\theta)\right)\right\} .
\end{aligned}
$$

A maximização directa de (4.3) pode ser difícil. Por esta razão, desenvolvemos o algoritmo EM a fim de obter um procedimento de estimação mais eficiente.

O algoritmo EM é uma ferramenta bem conhecida para a estimação de MV quando os dados não observados ou variáveis latentes são apresentados na modelagem (Dempster et al. [1977]). Seja $T_{1}, \ldots, T_{n}$ uma amostra aleátoria de $T \sim R L(\theta, \sigma)$. Aqui, o vector de parâmetros é $\boldsymbol{\psi}=(\theta, \sigma) \in \mathbb{R}_{+}^{2}$. Sejam $\ell_{c}\left(\boldsymbol{\psi} \mid \boldsymbol{t}_{c}\right)$ e $Q(\boldsymbol{\psi} \mid \widehat{\boldsymbol{\psi}})=\mathbb{E}\left[\ell_{c}\left(\boldsymbol{\psi} \mid \boldsymbol{t}_{c}\right) \mid \boldsymbol{t}_{c}, \widehat{\boldsymbol{\psi}}\right]$ denotando a função de log-verossimilhança completa e seu valor esperado condicionado, respectivamente. Sejam $\boldsymbol{t}=\left(t_{1}, \ldots, t_{n}\right)^{\top}, \boldsymbol{m}=\left(m_{1}, \ldots, m_{n}\right)^{\top}$ e $\boldsymbol{t}_{c}=\left(\boldsymbol{t}^{\top}, \boldsymbol{m}^{\top}\right)^{\top}$ os dados observados, não observados e completos, respectivamente. Agora escreveremos detalhadamente a implementação da estimação de MV para os parâmetros de distribuição RL utilizando o algoritmo EM. Usando a representação hierárquica a função log-verosimilhança completa para o modelo, a menos de uma constante, é dada por

$$
\ell_{c}\left(\boldsymbol{\psi} \mid \boldsymbol{t}_{c}\right)=-\sum_{i=1}^{n}\left(\frac{m_{i} t_{i}^{2}}{2 \sigma^{2}}+2 \delta_{i} \log \sigma\right)+\ell_{c}\left(\theta \mid \boldsymbol{t}_{c}\right),
$$


em que $\ell_{c}\left(\theta \mid \boldsymbol{t}_{c}\right)=-n \log (1+\theta)+\sum_{i=1}^{n}\left[\left(m_{i}-1\right) \log p+\log \left[\theta(1-2 p)+(1-p)\left(1+\theta\left(m_{i}-1\right)\right)\right]\right]$. Seja $\widehat{m}_{i}=\mathbb{E}\left[M_{i} \mid t_{i}, \psi=\widehat{\psi}\right]$. Com esta notação, temos que

$$
Q(\boldsymbol{\psi} \mid \widehat{\boldsymbol{\psi}})=-\sum_{i=1}^{n}\left(\frac{\widehat{m}_{i} t_{i}^{2}}{2 \sigma^{2}}+2 \delta_{i} \log \sigma\right)+Q(\theta \mid \widehat{\theta}),
$$

em que $Q(\theta \mid \widehat{\theta})=-n \log (1+\theta)-\theta \sum_{i=1}^{n}\left[\left(\widehat{m}_{i}-1\right)+W_{i}(\theta)\right]$ e $W_{i}(\theta)=\mathbb{E}[\log [\theta(1-2 p)+(1-$ $\left.\left.p)\left(1+\theta\left(M_{i}-1\right)\right)\right] \mid \boldsymbol{t}_{c}\right]$. Note-se que a maximização de $Q(\boldsymbol{\psi} \mid \widehat{\psi})$ é direta em relação a $\sigma$. Por outro lado, para $W_{i}(\theta)$ podemos usar uma abordagem semelhante à de Lee e Xu [2004]. Especificamente, seja $\left\{m_{r}, r=1, \ldots, R\right\}$ uma amostra aleatória da distribuição condicional $M_{i} \mid T_{i}=t_{i}, \boldsymbol{\psi}=\widehat{\boldsymbol{\psi}}$, de modo que a quantidade $W_{i}(\theta)$ pode ser aproximada como segue:

$$
W_{i}(\theta) \approx \frac{1}{R} \sum_{r=1}^{R} \log \left[\theta(1-2 p)+(1-p)\left(1+\theta\left(m_{r}-1\right)\right)\right] .
$$

Por outro lado, depois de algumas manipulações algébricas, é possível provar que para $i=1, \ldots, n$,

$$
\mathbb{E}\left[M_{i} \mid t_{i}, \delta_{i}, \boldsymbol{\psi}\right]= \begin{cases}\frac{p^{2} s_{i}-p\left(s_{i}(1-\theta)+2 \theta+1\right)+1+\theta}{\left(1-p s_{i}\right)\left[p^{2} s_{i}(1+\theta)-p\left(s_{i}+2 \theta+1\right)+1+\theta\right]} & , \text { se } \delta_{i}=0, \\ \frac{p^{3} s_{i}^{2}-p^{2} s_{i}\left(s_{i}(1-\theta)+4 \theta\right)+p\left(4 s_{i} \theta-2 \theta-1\right)+1+\theta}{\left(1-p s_{i}\right)\left[p^{2} s_{i}-p\left(s_{i}(1-\theta)+2 \theta+1\right)+1+\theta\right]} & , \text { se } \delta_{i}=1,\end{cases}
$$

em que $s_{i}=\exp \left\{-\frac{t_{i}^{2}}{2 \sigma^{2}}\right\}$. Note que (4.4) implica que $\widehat{m}_{i}$ é facilmente calculável. Em resumo, o algoritmo EM é implementado usando os seguintes passos:

- Passo-E: Dado $\widehat{\boldsymbol{\psi}}=\widehat{\boldsymbol{\psi}}^{(k)}$, calcular $\widehat{m}_{i}^{(k+1)}=\mathbb{E}\left[M_{i} \mid t_{i}, \delta_{i}, \boldsymbol{\psi}^{(k+1)}\right]$ dado em (4.4).

- Passo-CM I: Atualize o parâmetro $\sigma$ como

$$
\sigma^{(k+1)}=\sqrt{\frac{\sum_{i=1}^{n} \widehat{m}_{i}^{(k+1)} t_{i}^{2}}{2 \sum_{i=1}^{n} \delta_{i}}} .
$$

e supondo que existe pelo menos uma falha.

- Passo-CM II: Atualize o parâmetro $\theta$ como

$$
\theta^{(k+1)}=\arg \max _{\theta}\left[-n \log (1+\theta)-\theta \sum_{i=1}^{n}\left(\widehat{m}_{i}^{(k+1)}-1+W_{i}(\theta)\right)\right] .
$$

As iterações são repetidas até que uma regra de convergência adequada é satisfeita. Por exemplo, $\left|\ell\left(\boldsymbol{\psi}^{(k+1)}\right)-\ell\left(\boldsymbol{\psi}^{(k)}\right)\right|$ é suficientemente pequeno. Como mostrado na Observação 5 , o modelo Rayleigh é um caso particular quando $\theta \rightarrow \infty$. Por esta razão, pode-se fixar $\widehat{\theta}$ num valor elevado (digamos $\theta(0)=1$ ) e $\sigma(0)=\sqrt{\overline{t^{2}}}$ (o EMV para $\sigma$ no modelo Rayleigh) como os valores iniciais para o algoritmo. Finalmente, os erros padrões podem ser estimados com base na matriz hessiana.

Observação 8 O passo-CM II pode ser muito lento. Uma alternativa é usar a ideia em Lin et al. [2007] e substituir o Passo-CM II seguinte por:

- Passo-CML II: Atualizar $\theta^{(k+1)}$ otimizando a seguinte função de log-verossimilhança perfilada:

$$
\theta^{(k+1)}=\arg \max _{\theta} \ell\left(\theta, \sigma^{(k+1)}\right) .
$$


- Note que para $\delta_{i}=1, i=1, \ldots n$, é recuperado o caso quando todas as observações são não censuradas(completas).

\subsection{Estudo de simulação}

Nesta seção, apresentamos um estudo de simulação para avaliar o procedimento de estimação proposto com base ao algoritmo EM, dado pela Seção 4.4.2. Para simular um valor da distribuição $\mathrm{RL}$, usamos o seguinte algoritmo:

- Passo 1: Simular $M \sim D L(\theta)$ se $M^{*} \sim L(\theta)$, então, $M=\left\lfloor M^{*}\right\rfloor+1 \sim L D(\theta)$ no pacote VGAM em $\mathrm{R}$ (permite simular valores da distribuição Lindley).

- Passo 2: Simular $W_{1}, \ldots, W_{M} \sim R(\sigma)$ (se $U \sim U(0,1)$, então $\sqrt{-2 \sigma^{2} \log U} \sim R(\sigma)$ ).

- Passo 3: Definir $T=\min \left(W_{1}, \ldots, W_{M}\right)$.

Por construção $T \sim L R(\theta, \sigma)$. Realizamos um estudo de simulação considerando os seguintes casos:

- $\theta \in\{0.25,0.5,0.75,1.0\}$;

- O paramêtro de escala $\sigma \in\{1,5,10\}$;

- O tamanho da amostra $n \in\{20,30,50,75,100,200\}$.

Para cada combinação de $(\theta, \sigma, n)$, simulamos 1000 réplicas e para cada uma, calculamos (se possível) a estimativa de máxima verossimilhança(usando o algoritmo EM apresentado) e os estimadores de momentos. Apresentamos o viés para tais estimadores, os desvios padrões para os estimadores de momentos, a média dos erros padrão estimados dos EMV com base na matriz hessiana observada, a raiz do erro quadrado médio $(\sqrt{E Q M})$ para os EMV e a proporção de vezes em que não existem (prop.n.e.) e os estimadores de momentos. As Tabelas 4.1 e 4.2 mostram os resultados que ilustram o comportamento dos EMV e os estimadores de momentos. Como podemos ver, os resultados são satisfatórios entre as combinações de parâmetros considerados. Como esperado, a medida que o tamanho da amostra aumenta as estimativas são mais próximos ao valor verdadeiro de $\sigma$ e $\theta$. Além disso, desvios-padrão e a $\sqrt{E Q M}$ estão mais próximos. Nota-se a partir dos estudos de simulação que os EMV's de $\sigma$ e $\theta$ apresentam menores vieses e erros padrões estimados do que os correspondentes valores obtidos com os métodos de momentos. Observamos que os resultados obtidos são aceitáveis, mesmo para pequenas amostras ( $n=20$ para EMV's e $n=30$ para os estimadores de momentos). 


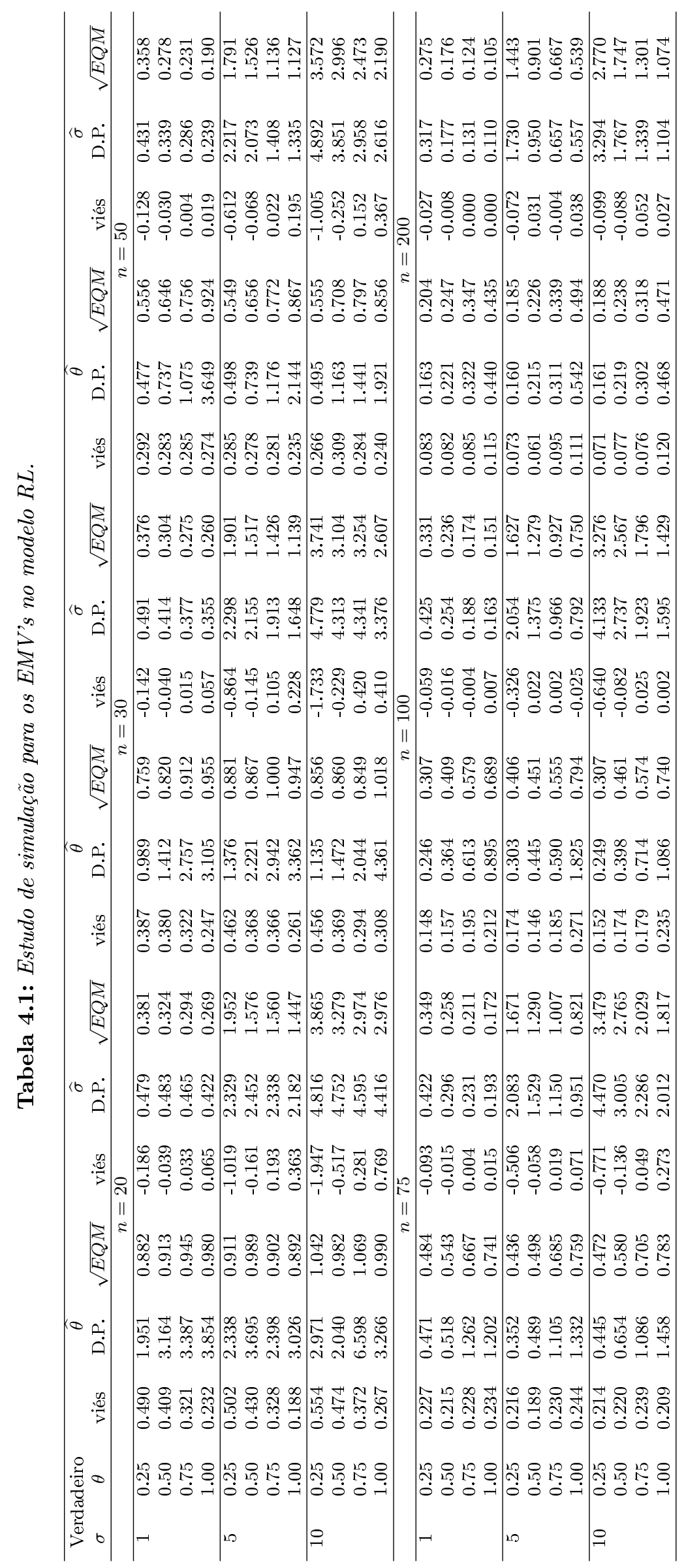




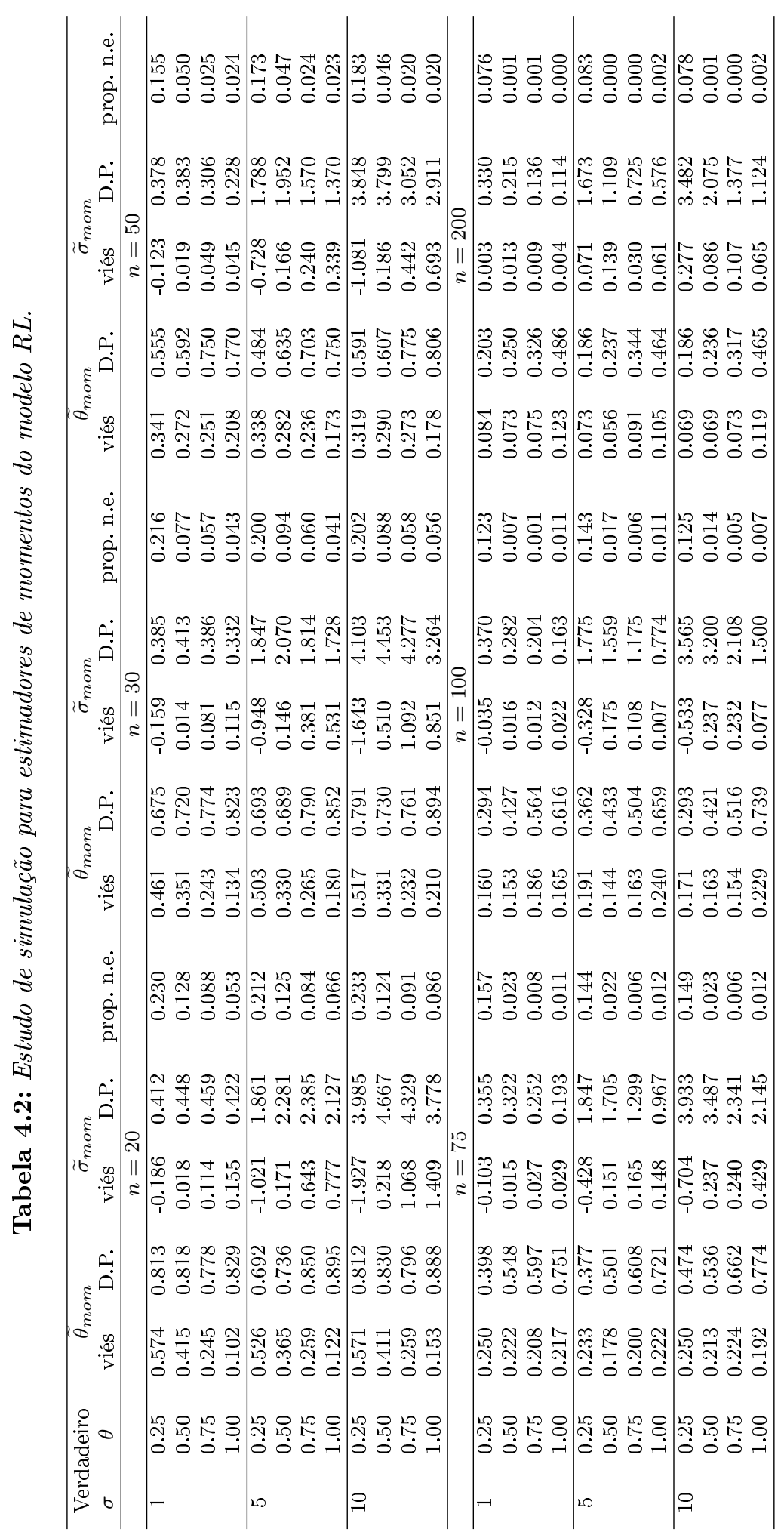




\subsection{Ilustração}

Nesta seção analisamos dois conjuntos de dados usando as distribuições de Rayleigh e RayleighLindley. Além disso, analisamos os conjuntos de dados usando dois modelos parcimoniosos, incluindo um parâmetro de forma e um de escala, que são as distribuições Weibull (W) e gama (G). Estas distribuições são geralmente utilizadas para analisar este tipo de conjunto de dados.

\subsubsection{Tempos de sobrevivência de cobaias}

O seguinte conjunto de dados foi previamente analisado em Bjerkedal [1960], Gupta et al. [1997] e Kundu et al. [2008]. Os dados representam os tempos de sobrevivência de cobaias (em dias) injectados com doses diferentes de bacilos da tuberculose. O conjunto de dados completos encontrase no Apêndice A.1

Na Tabela 4.3 apresentamos as estatísticas descritivas para os tempos de sobrevivência de cobaias.

Tabela 4.3: Estatísticas descritivas para os tempos de sobrevivência de cobaias.

\begin{tabular}{ccccc}
\hline$n$ & $\bar{t}$ & $s^{2}$ & $\sqrt{b_{1}}$ & $b_{2}$ \\
\hline 72 & 99.819 & 6580.122 & 1.758 & 2.459 \\
\hline
\end{tabular}

Na Tabela 4.4 mostramos as estimativas para todos os modelos considerados. Note-se que os critérios AIC e BIC sugerem que a distribuição RL fornece um melhor ajuste para este conjunto de dados. Além disso, também aplicamos três testes de bondade de ajuste vistos no Capítulo 2. Os resultados são apresentados na Tabela 4.5. Note-se que os três testes de bondade de ajuste dizem que os dados vieram da distribuição RL. Finalmente, na Figura 4.3 apresenta-se a fda para os modelos ajustados.

Tabela 4.4: Estimativas dos parâmetros, desvíos padrões(d.p.) e valor da função log-verossimilhança para os modelos $R, W, G$ e RL para os tempos de sobrevivência de cobaias.

\begin{tabular}{ccccc}
\hline Parâmetros & R (d.p.) & W (d.p.) & G (d.p.) & RL (d.p.) \\
\hline$\sigma$ & $90.699(5.344)$ & $110.555(9.934)$ & $47.956(8.409)$ & $188.258(71.719)$ \\
$\theta$ & - & $1.393(0.118)$ & $2.081(0.323)$ & $0.198(0.152)$ \\
\hline AIC & 818.592 & 798.295 & 792.495 & 787.206 \\
BIC & 820.869 & 802.849 & 797.048 & 791.760 \\
\hline
\end{tabular}

Tabela 4.5: Testes de bondade de ajustes para o modelo RL.

\begin{tabular}{cccc}
\hline Critério & KS & AD & CVM \\
\hline Observado & 0.098 & 1.122 & 0.177 \\
Valor-p & 0.497 & 0.299 & 0.317 \\
\hline
\end{tabular}

\subsubsection{Tempos de falha de componentes}

Este conjunto de dados corresponde a tempos de falha de 50 componentes (medidos em unidades de 1.000 horas). O conjunto de dados completo é tomado a partir de Murthy et al. [2004], página 195, conjunto de dados 9.2. O conjunto de dados completo é dado no Apêndice A.1

Na Tabela 4.6 apresentamos as estatísticas descritivas para o conjunto de dados dos tempos de falha, em que é possível observar que os dados apresentan uma curtose alta e pela Figura 4.4, que existe presença se observações discrepantes. 


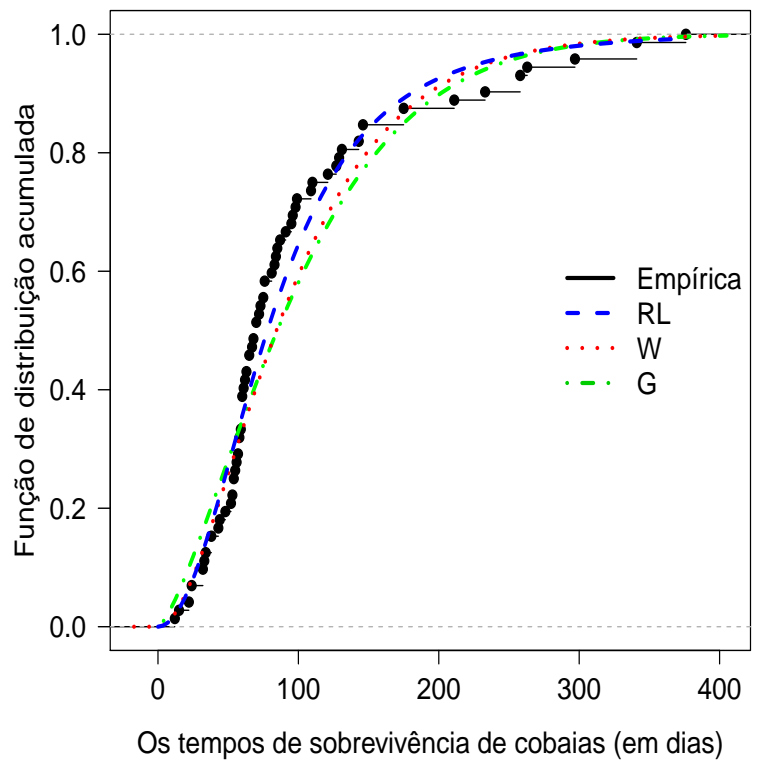

Figura 4.3: Função de distribuição acumulada para os modelos ajustados aos dados dos tempos de sobrevivência de cobaias.

Tabela 4.6: Estatisticas descritivas para o conjunto de dados dos tempos de falha de componentes.

\begin{tabular}{ccccc}
\hline$n$ & $\bar{t}$ & $s^{2}$ & $\sqrt{b_{1}}$ & $b_{2}$ \\
\hline 50 & 0.2949 & 0.072 & 1.824 & 5.905 \\
\hline
\end{tabular}
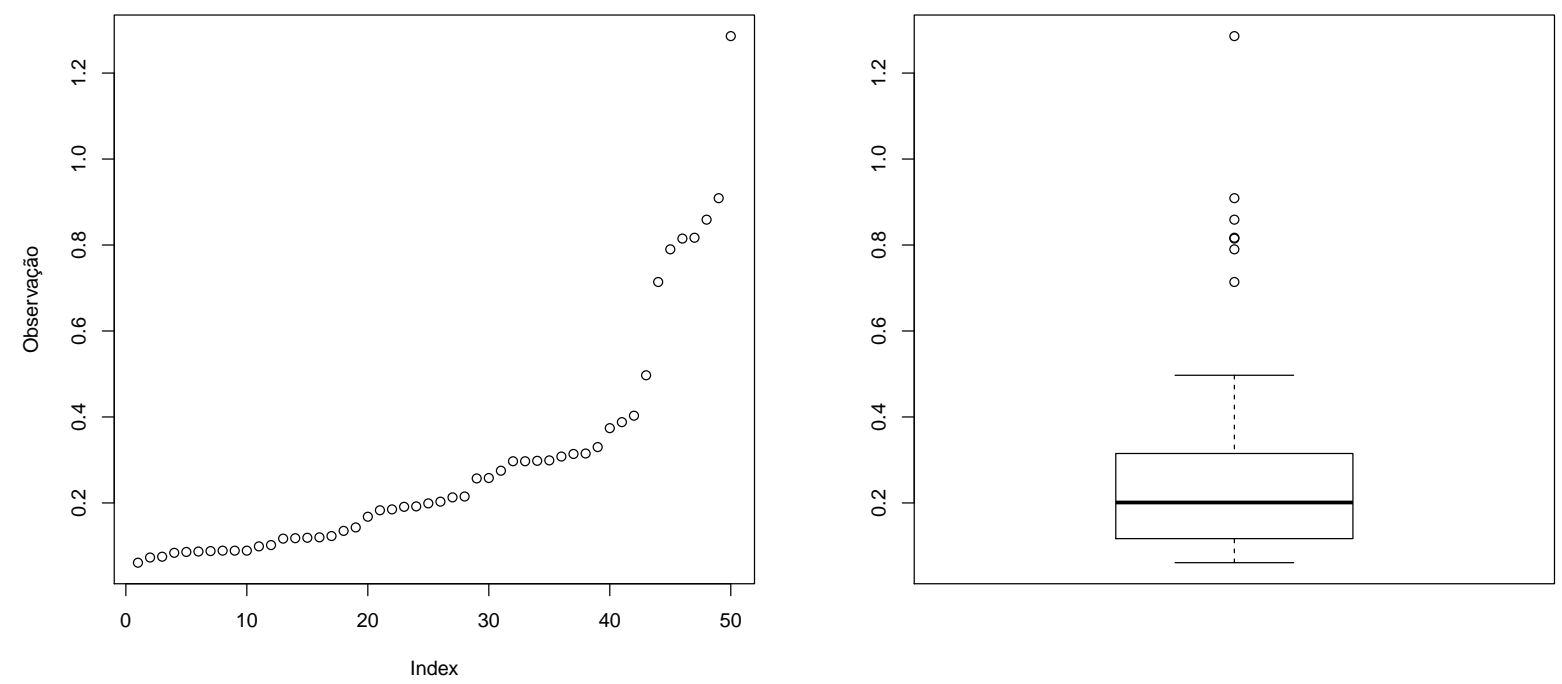

Figura 4.4: Graficos de dispersão e boxplot dos dados.

A Tabela 4.7 mostra as estimativas dos parâmetros para os mesmos modelos considerados no último exemplo. Nota-se que os critérios AIC e BIC sugerem que RL fornece melhor ajuste para estes dados que os modelos R, W e G. Adicionalmente, a Tabela 4.8 mostra os testes de bondade de ajuste para este conjunto de dados. Os três testes de bondade de ajuste sugerem que os dados vieram da distribuição RL. Finalmente, a Figura 4.5 mostra as funções de densidade ajustadas com 
o histograma deste conjunto de dados, em que a linha tracejada em azul é do modelo RL.

Tabela 4.7: Estimativas dos parâmetros e os valores da log-verossimilhança para os modelos $R, W, G$ e $R L$ para o conjunto de dados dos tempos de falha.

\begin{tabular}{ccccc}
\hline Parâmetros & R (d.p.) & W (d.p.) & G (d.p.) & RL (d.p.) \\
\hline$\sigma$ & $0.281(0.020)$ & $0.320(0.038)$ & $0.172(0.037)$ & $0.605(0.264)$ \\
$\theta$ & - & $1.258(0.129)$ & $1.719(0.316)$ & $0.155(0.135)$ \\
\hline AIC & 1.948 & -22.495 & -25.618 & -28.159 \\
BIC & 3.860 & -18.671 & -21.794 & -24.335 \\
\hline
\end{tabular}

Tabela 4.8: Testes de bondade de ajuste para o modelos RL para o conjunto de dados dos tempos de falha.

\begin{tabular}{cccc}
\hline Critério & KS & AD & CVM \\
\hline Observado & 0.124 & 1.273 & 0.136 \\
Valor-p & 0.428 & 0.241 & 0.435 \\
\hline
\end{tabular}

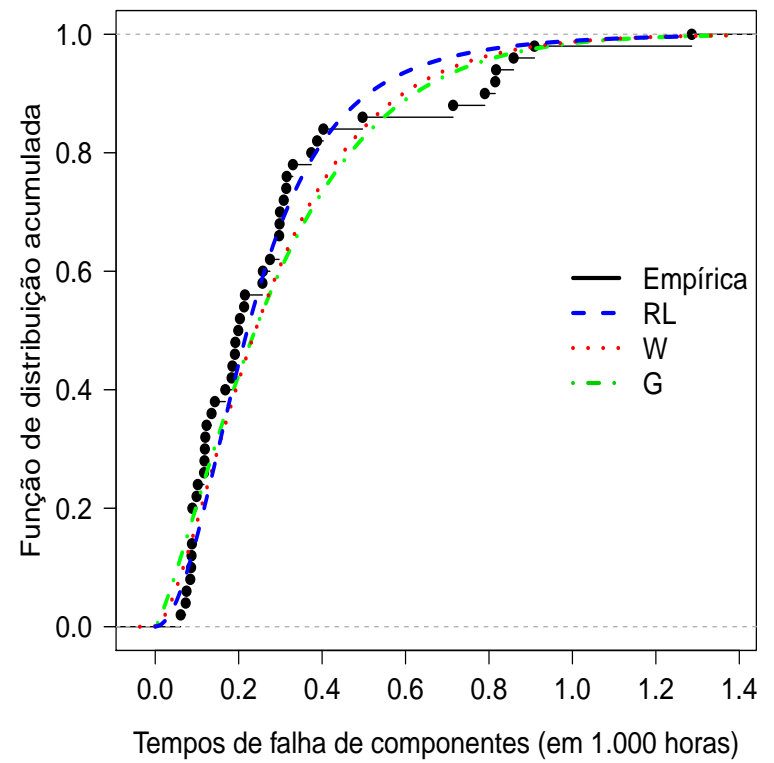

Figura 4.5: Função de distribuição acumulada para os modelos ajustados no conjunto de dados de tempos de falhas.

\subsection{Observações finais}

Neste capítulo é introduzida a distribuição Rayleigh-Lindley (RL), que é obtida compondo as distribuições Rayleigh e a Lindley discreta. Esta nova distribuição pode ser usada como uma alternativa para os modelos Weibull e gama já que é parcimoniosa, pois contém só dois parâmetros. Foi feito um estudo de simulação para comparar o comportamento dos estimadores de máxima verossimilhança e momentos, em que se verifica que para pequenos tamanhos amostrais, digamos $n=20$ o EMV's são mais próximos dos verdadeiros valores. Finalmente na aplicação, aos dois conjuntos de dados analisados, o modelo RL mostrou um melhor ajuste segundo os critérios AIC e BIC, quando é comparado com os modelos gama, Weibull e Rayleigh. 


\section{Capítulo 5}

\section{Distribuição exponencial segmentada potência}

Neste capítulo uma extensão da distribuição exponencial segmentada com base na distribuição do máximo de uma amostra aleatória é considerada. Propriedades de sua função densidade e su função de risco são investigadas. Inferência por máxima verosimilhança é discutida e a matriz de informação de Fisher é obtida. Os resultados de duas aplicaçôes a dados reais são relatados, em que o modelo é ajustado usando máxima verosimilhança. As aplicações ilustram o melhor desempenho da nova distribuição quando comparada com modelos alternativos recentemente propostos na literatura.

\subsection{Motivação}

Lehmann [1953] propôs uma família de distribuições, cuja distribuição acumulada $F(z)$, é a potência racional de outra função de distribuição acumulada continua e diferenciável $F_{0}(z)$, na literatura este é conhecido como modelo de alternativas de Lehmann. Este modelo é dado por

$$
\mathcal{F}_{F_{0}}(z ; \alpha)=\left\{F_{0}(z)\right\}^{\alpha}, \quad z \in \mathbb{R}
$$

em que $F_{0}$ é uma fda e $\alpha$ é um inteiro ou um número racional. Devido ao seu papel central nessa construção, vamos referir-nos $F_{0}$ como o núcleo. Este modelo tem sido discutido amplamente por Arnold [2004] e Gupta e Gupta [2008]. Durrans [1992], num contexto hidrológico, deriva este modelo como uma distribuição de estatística de ordem fracionária, que nós chamamos de distribuições potência.

Sem referenciar Lehmann (1953), Durrans [1992] estendeu a expressão (5.1) permitindo $\alpha$ variar ao longo do conjunto $\mathbb{R}^{+}$, referindo-se as distribuições resultantes como sendo de estatísticas de ordem fracionárias. Assumindo $F_{0}$ absolutamente contínua com a densidade $f_{0}$ nesta extensão de (5.1), a função densidade de uma variável aleatória, $Z$, com essa distribuição é dada por

$$
\varphi_{F_{0}}(z ; \alpha)=\alpha f_{0}(z)\left\{F_{0}(z)\right\}^{\alpha-1}, \quad z \in \mathbb{R}, \quad \alpha \in \mathbb{R}^{+} .
$$

Escrevemos $Z \sim F O_{F_{0}}(\alpha)$ neste caso.

Durrans [1992] considerou o caso em que $F_{0}$ em (5.2) é a distribuição normal padrão, assim a fdp é

$$
\varphi_{\Phi}(z ; \alpha)=\alpha \phi(z)\{\Phi(z)\}^{\alpha-1}, \quad z \in \mathbb{R}, \quad \alpha \in \mathbb{R}^{+},
$$

e foi denominada como a distribuição gaussiana generalizada. Gupta e Gupta [2008] também considerarum a distribuição $F O_{\Phi}(\alpha)$ com mais detalhes. Eles se referiram a Lehmann [1953], mas aparentemente não tinham conhecimento do trabalho de Durrans [1992]. Eles se referiam à classe de distribuições com densidade (5.3) como o modelo normal potência. Para mais detalhes sobre (5.3), vide Pewsey et al. [2012].

Mais geralmente, será adequado considerar modelos de potência, ou alternativas de Lehmann, que envolvem uma família de funções de distribuição paramétrica no núcleo. Assim, começaremos com uma família paramétrica, $\left\{F_{0}(x ; \boldsymbol{\theta}): \boldsymbol{\theta} \in \boldsymbol{\Theta}\right\}$, de distribuições e considerar um modelo mais flexível da forma

$$
F(x ; \boldsymbol{\theta}, \alpha)=\left[F_{0}(x ; \boldsymbol{\theta})\right]^{\alpha} .
$$

Neste capítulo, consideraremos os modelos do tipo (5.4). Com aplicações para a confiabilidade 
em mente, consideraremos o caso particular do modelo (5.4) no qual a família de distribuições do núcleo corresponde à distribuição exponencial segmentada. Este modelo será apropriado quando as taxas de falhas sofrem mudanças bruscas em horários específicos devido, normalmente, a mudanças ambientais.

O modelo exponencial segmentado (MES), apresentado no Capítulo 2, surge como uma alternativa muito atraente aos modelos paramétricos usados na análise de tempos de vida. Embora paramétrico em sentido estrito, o MES pode ser pensado como um modelo semiparamétrico.Esta característica agradável do modelo ES é que ele nos permite aproximar funções de risco das mais variadas formas de modo bastante satisfatório.

Este capítulo está focado no estudo da distribuição que é gerada quando se considera a família de distribuições (5.2) com a distribuição exponencial segmentada. A distribuição resultante é o modelo exponencial segmentado potência (ESP), com função de risco mais flexível do que o modelo exponencial segmentada (ES) para o qual a função de risco é constante nos intervalos e diferente entre intervalos. Esta flexibilização permite que a função de risco nos intervalos possa assumir diferentes formas (inclusive banheira descontínua). A forma da função de risco em cada intervalo é controlada pelo parâmetro $\alpha$.

A motivação para a introdução desta nova distribuição surge a partir de um desejo de ter um modelo com um comportamento diferente da função de risco nos sub-intervalos de $(0, \infty)$, mas não necessariamente constante como é o caso com a distribuição ES. A introdução de um único parâmetro potência, $\alpha$, conduzindo à distribuição ESP atinge este objetivo em certa medida. A função de risco correspondente é diferente e não é constante nos diferentes sub-intervalos. O modelo de ESP com sua variedade relativamente parcimoniosa dos parâmetros (apenas um a mais do que o modelo ES), por vezes, vai servir como uma extensão bastante adequada para o ajuste de conjuntos de dados. Situações práticas em que a modelagem por partes é mais adequada abundam na literatura. A distribuição ESP discutida neste capítulo herda uma estrutura por partes da distribuição ES, mas já não tem uma suposição muitas vezes irrealista de taxas de risco que são constantes nos sub-intervalos.

A distribuição ESP resultante com pelo menos dois parâmetros (dependendo do numero intervalos) pode ser utilizada para ajustar dados resultantes de experimentos na teoria da confiabilidade tempos e tempos de vida.

Este modelo pode ser usado para dados positivos de confiabilidade ou de sobrevivência e é, portanto, uma alternativa viável para modelos tais como half-normal, exponencial, gama e Weibull, entre outros.

\subsection{Modelo exponencial segmentado potência}

Definição 5.2.1 Usando a equação (5.2) a função de distribuição do modelo exponencial segmentado, obtemos a distribuição exponencial segmentada potência com os parâmetros $\boldsymbol{\lambda}$ e $\alpha$ e os tempos de partição a, denotado por $\operatorname{ESP}(\boldsymbol{\lambda}, \alpha, \boldsymbol{a})$, é

$$
F(t ; \boldsymbol{\lambda}, \alpha, \boldsymbol{a})=\left[F_{0}(t ; \boldsymbol{\lambda}, \boldsymbol{a})\right]^{\alpha}, \quad t>0,
$$

em que $F_{0}(\cdot ; \boldsymbol{\lambda}, \boldsymbol{a})$ é a fda da distribuição exponencial segmentada, igual $1-S_{0}(\cdot ; \boldsymbol{\lambda}, \boldsymbol{a})$ descrita nas equações (2.4)-(2.7). A função de densidade é dada por

$$
f(t ; \boldsymbol{\lambda}, \alpha, \boldsymbol{a})=\alpha\left[F_{0}(t ; \boldsymbol{\lambda}, \boldsymbol{a})\right]^{\alpha-1} f_{0}(t ; \boldsymbol{\lambda}, \boldsymbol{a}), \quad t>0,
$$

em que $f_{0}(\cdot ; \boldsymbol{\lambda}, \boldsymbol{a})$ é a $f d p$ da distribuição exponencial segmentada, como em (2.5).

\subsubsection{Principais propriedades}

A função de risco é calculada como $h(t \mid \boldsymbol{\lambda}, \alpha, \boldsymbol{a})=f(t \mid \boldsymbol{\lambda}, \alpha, \boldsymbol{a}) /[1-F(t \mid \boldsymbol{\lambda}, \alpha, \boldsymbol{a})]$. Esta função de risco é segmentada, mas não admite uma representação simples. A Figura 5.1 mostra a função de densidade e de risco com $L=2$ e uma partição do tempo de $a_{1}=2$ (um caso em que um único ponto 
de mudança está envolvido) para alguns valores dos parâmetros $\lambda_{1}, \lambda_{2}$ e $\alpha$. A Figura 5.1 mostra a flexibilidade do modelo proposto, apresentando formas diferentes nos intervalos, dependendo dos valores dos parâmetros selecionados. A função de risco pode assumir três formas em cada intervalo: crescente $(\alpha>1)$, decrescente $(\alpha<1)$ e constante $(\alpha=1)$. O último caso corresponde ao modelo ES tradicional.
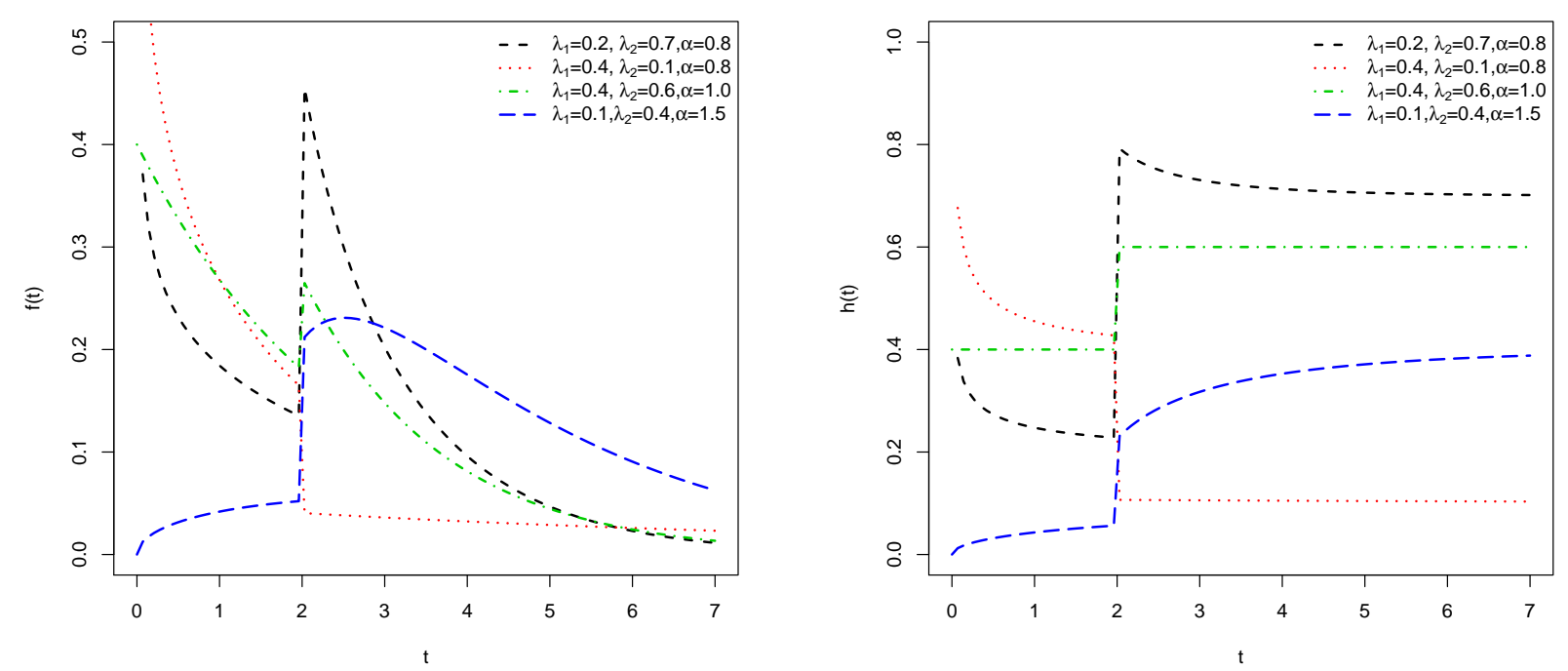

Figura 5.1: Função de densidade (esquerda) e função de risco (direita) para uma partição de tempo em que $L=2$ e $a_{1}=2$ para vários valores de $\lambda_{1}, \lambda_{2}$ e $\alpha$.

Casos particulares do modelo de ESP são os seguintes:

- $\alpha=1$ : modelo exponencial segmentado, [Friedman , 1982]

- $L=1$ : modelo exponencial exponencializado, [Gupta e Kundu, 2001]

- $\alpha=1$ e $L=1$ :modelo exponencial.

Proposição 5.2.1 Se $T \sim E S P(\boldsymbol{\lambda}, \alpha, \boldsymbol{a})$, então seus momentos assumem a forma

$$
\begin{gathered}
E\left(T^{n}\right)=\alpha \sum_{\ell=1}^{L} \lambda_{\ell}^{-n} \sum_{k=0}^{\infty}\left(\begin{array}{c}
\alpha-1 \\
k
\end{array}\right) \frac{(-1)^{k}}{(k+1)^{n+1}} \kappa_{\ell}^{k+1} \exp \left\{(k+1) \lambda_{\ell} a_{\ell-1}\right\} \\
\times\left[\Gamma\left(n+1,(k+1) \lambda_{\ell} a_{\ell-1}\right)-\Gamma\left(n+1,(k+1) \lambda_{\ell} a_{\ell}\right)\right],
\end{gathered}
$$

em que $\left(\begin{array}{l}\alpha \\ k\end{array}\right)$ é o coeficiente binomial generalizado definido como $\left(\begin{array}{l}\alpha \\ k\end{array}\right)=\prod_{i=1}^{k}(\alpha-i+1) / \prod_{i=1}^{k}(k-i+1)$ $e \Gamma(n, x)=\int_{x}^{\infty} u^{n-1} e^{-u} d u$ é a função gama incompleta (superior).

Prova Por definição,

$$
\begin{aligned}
E\left(T^{n}\right) & =\int_{0}^{\infty} t^{n} f(t ; \boldsymbol{\lambda}, \alpha, \boldsymbol{a}) d t \\
& =\alpha \sum_{\ell=1}^{L} \int_{a_{\ell-1}}^{a_{\ell}} t^{n} \kappa_{\ell} \lambda_{\ell} \exp \left\{-\lambda_{\ell}\left(t-a_{\ell-1}\right)\right\}\left[1-\exp \left\{-\sum_{d=1}^{L} \lambda_{d} \nabla_{d}(t)\right\}\right]^{\alpha-1} d t .
\end{aligned}
$$


Usando a identidade $(1+b)^{c}=\sum_{k=0}^{\infty}\left(\begin{array}{l}c \\ k\end{array}\right) b^{k}$ (note que se $c$ é um número inteiro haverá apenas $c+1$ termos diferentes de zero nesta expansão) e a definição de $\nabla_{d}(t)$ em $(2.7)$, temos que

$$
\begin{aligned}
E\left(T^{n}\right)= & \alpha \sum_{\ell=1}^{L} \kappa_{\ell} \lambda_{\ell} \sum_{k=0}^{\infty}\left(\begin{array}{c}
\alpha-1 \\
k
\end{array}\right)(-1)^{k} \exp \left\{-I(\ell>1) k \sum_{d=1}^{\ell-1} \lambda_{d}\left(a_{d}-a_{d-1}\right)+(k+1) \lambda_{\ell} a_{\ell-1}\right\} \\
& \times \int_{a_{\ell-1}}^{a_{\ell}} t^{n} \exp \left\{-(k+1) \lambda_{\ell} t\right\} d t \\
= & \alpha \sum_{\ell=1}^{L} \lambda_{\ell} \sum_{k=0}^{\infty}\left(\begin{array}{c}
\alpha-1 \\
k
\end{array}\right)(-1)^{k} \kappa_{\ell}^{k+1} \exp \left\{(k+1) \lambda_{\ell} a_{\ell-1}\right\} \int_{a_{\ell-1}}^{a_{\ell}} t^{n} \exp \left\{-(k+1) \lambda_{\ell} t\right\} d t .
\end{aligned}
$$

Usando a mudança $w=(k+1) \lambda_{\ell} t$, temos que a última integral assume a forma

$$
\begin{aligned}
\int_{a_{\ell-1}}^{a_{\ell}} t^{n} \exp \left\{-(k+1) \lambda_{\ell} t\right\} d t & =\frac{1}{(k+1)^{n+1} \lambda_{\ell}^{n+1}} \int_{(k+1) \lambda_{\ell} a_{\ell-1}}^{(k+1) \lambda_{\ell} a_{\ell}} w^{n} e^{-w} d w \\
& =\frac{1}{(k+1)^{n+1} \lambda_{\ell}^{n+1}}\left[\Gamma\left(n+1,(k+1) \lambda_{\ell} a_{\ell-1}\right)-\Gamma\left(n+1,(k+1) \lambda_{\ell} a_{\ell}\right)\right] .
\end{aligned}
$$

Substituindo o resultado de (5.9) na integral em (5.8) o resultado é obtido.

A Proposição 5.2.1 pode ser usada para calcular qualquer momento desejado dado um conjunto de valores para os parâmetros de $\boldsymbol{\lambda}$ e $\alpha$, o parâmetros de tempo de partição $L$ e $\boldsymbol{a}$. Em particular, temos o seguinte corolário.

\section{Corolário 5.2.1}

$$
E(T)=\alpha \sum_{\ell=1}^{L} \lambda_{\ell}^{-1} \sum_{k=0}^{\infty}\left(\begin{array}{c}
\alpha-1 \\
k
\end{array}\right) \frac{\left(-\kappa_{\ell}\right)^{k+1}}{(k+1)^{2}}\left[\left\{(k+1) \lambda_{\ell} a_{\ell}+1\right\} e^{-(k+1) \lambda_{\ell}\left(a_{\ell}-a_{\ell-1}\right)}-\left\{(k+1) \lambda_{\ell} a_{\ell-1}+1\right\}\right] .
$$

A Figura 5.2 mostra a média, a variância e os coeficientes de assimetria e curtose em função do parâmetro $\alpha$, para alguns valores dos parâmetros $\lambda_{1}$ e $\lambda_{2}$. Nas figuras $L=2$ e $a_{1}=2$.

A Figura 5.2 indica que as medidas de assimetria e curtose como funções de $\alpha$ podem assumir formas diferentes, dependendo dos valores dos parâmetros de intensidade $\lambda_{l}$ 's.

Proposição 5.2.2 Se $T \sim E S P(\boldsymbol{\lambda}, \alpha, \boldsymbol{a})$ a sua função geradora de momentos assume a forma

$$
M_{T}(u)=\alpha \sum_{\ell=1}^{L} \lambda_{\ell}^{-n} \sum_{k=0}^{\infty}\left(\begin{array}{c}
\alpha-1 \\
k
\end{array}\right) \frac{(-1)^{k} \kappa_{\ell}^{k+1}\left[\exp \left\{u a_{l}-\lambda_{l}(k+1)\left(a_{l}-a_{l-1}\right)\right\}-\exp \left\{u a_{l-1}\right\}\right]}{(k+1)^{n+1}\left(u-\lambda_{l}(k+1)\right)} .
$$

Prova Da mesma forma que a prova da Proposição 5.2.1, temos que

$$
\begin{aligned}
M_{T}(u)= & E\left(e^{T u}\right) \\
= & \alpha \sum_{\ell=1}^{L} \lambda_{\ell} \sum_{k=0}^{\infty}\left(\begin{array}{c}
\alpha-1 \\
k
\end{array}\right)(-1)^{k} \kappa_{\ell}^{k+1} \exp \left\{(k+1) \lambda_{\ell} a_{\ell-1}\right\} \\
& \times \int_{a_{\ell-1}}^{a_{\ell}} \exp \left\{-\left[(k+1) \lambda_{\ell}-u\right] t\right\} d t .
\end{aligned}
$$

Usando a mudança de variável $w=(k+1) \lambda_{\ell}-u$ na integral, o resultado é obtido. 

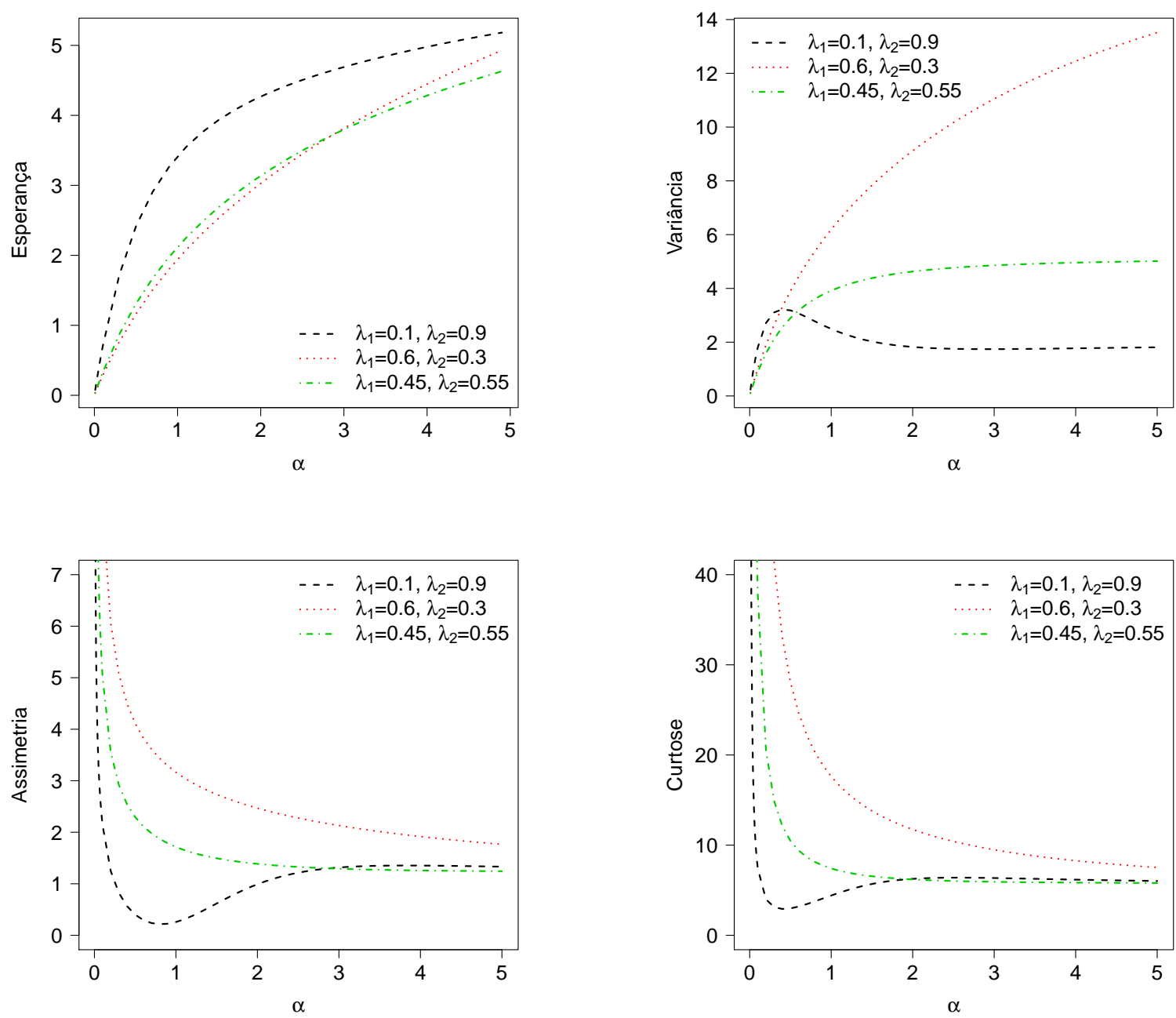

Figura 5.2: Algumas características da distribuição ESP para valores diferentes dos parâmetros. Painel superior esquerdo: média. Painel superior direito: variância. Painel inferior esquerdo: coeficiente de assimetria. Painel inferior direito: coeficiente de curtose. Em todos os casos $L=2$ e $a_{1}=2$.

\subsection{Inferência}

\subsubsection{A função de log-verossimilhança}

Dada uma amostra aleatória $t_{1}, \ldots, t_{n}$ da distribuição $\operatorname{ESP}(\boldsymbol{\lambda}, \alpha, \boldsymbol{a})$, em que $\boldsymbol{a}$ é um vector de partição de tempo conhecido, a função de log-verossimilhança para $\boldsymbol{\psi}=(\boldsymbol{\lambda}, \alpha)$ é dada por

$$
\begin{aligned}
l(\boldsymbol{\psi})= & n \log \alpha+(\alpha-1) \sum_{i=1}^{n} \log \left[1-\exp \left(-\sum_{l=1}^{L} \lambda_{l} \nabla_{l}\left(t_{i}\right)\right)\right]+\sum_{l=1}^{L} n_{l}\left(\log \lambda_{l}-\lambda_{l}\left(\bar{t}_{l}-a_{l-1}\right)\right) \\
& -\sum_{l=2}^{L} n_{l} \sum_{j=1}^{l-1} \lambda_{j}\left(a_{j}-a_{j-1}\right)
\end{aligned}
$$

em que $n_{l}$ e $\bar{t}_{l}$ são o número de observações e a média dos tempos observados no intervalo $\left(a_{l-1}, a_{l}\right)$ $\ell=1,2, \ldots L$, respectivamente.

\subsubsection{A função escore}

O vector escore é dado por $\mathbf{U}(\psi)=\left(\frac{\partial l(\psi)}{\partial \lambda_{1}}, \ldots, \frac{\partial l(\psi)}{\partial \lambda_{L}}, \frac{\partial l(\psi)}{\partial \alpha}\right)^{T}$, em que 


$$
\begin{aligned}
\frac{\partial l(\psi)}{\partial \lambda_{\ell}} & =(\alpha-1) \sum_{i=1}^{n} \frac{\nabla_{\ell}\left(t_{i}\right) e^{-u_{i}}}{1-e^{-u_{i}}}+n_{\ell}\left(\lambda_{\ell}^{-1}-\left(\bar{t}_{\ell}-a_{\ell-1}\right)\right)+\left(a_{\ell}-a_{\ell-1}\right) \sum_{l=\ell+1}^{L} n_{l}, \quad \ell=1, \ldots, L-1, \\
\frac{\partial l(\psi)}{\partial \lambda_{L}} & =(\alpha-1) \sum_{i=1}^{n} \frac{\nabla_{L}\left(t_{i}\right) e^{-u_{i}}}{1-e^{-u_{i}}}+n_{L}\left(\lambda_{L}^{-1}-\left(\bar{t}_{L}-a_{L-1}\right)\right) \\
e & \\
\frac{\partial l(\psi)}{\partial \alpha} & =\frac{n}{\alpha}+\sum_{i=1}^{n} \log \left(1-e^{-u_{i}}\right),
\end{aligned}
$$

em que $u_{i}=\sum_{l=1}^{L} \lambda_{l} \nabla_{l}\left(t_{i}\right)$. O estimador de máxima verossimilhança para $\boldsymbol{\psi}$, digamos $\widehat{\boldsymbol{\psi}}$, é obtido resolvendo a equação $\mathbf{U}(\boldsymbol{\psi})=0$. Esta solução pode ser obtida utilizando procedimentos numéricos tais como o algoritmo de Newton-Raphson.

\subsubsection{Matriz de informação observada}

A matriz de variâncias assintótica de $\widehat{\boldsymbol{\psi}}$ pode ser estimada por $\widehat{\operatorname{Var}(\widehat{\boldsymbol{\psi}}})=\{\boldsymbol{B}(\widehat{\boldsymbol{\psi}})\}^{-1}$, o inverso da matriz de informação observada, que é dada por

$$
-\boldsymbol{B}(\psi)=\left(\begin{array}{ccccc}
\frac{\partial^{2} l(\boldsymbol{\psi})}{\partial \lambda_{1}^{2}} & \frac{\partial^{2} l(\boldsymbol{\psi})}{\partial \lambda_{1} \partial \lambda_{2}} & \cdots & \frac{\partial^{2} l(\boldsymbol{\psi})}{\partial \lambda_{1} \partial \lambda_{L}} & \frac{\partial^{2} l(\boldsymbol{\psi})}{\partial \lambda_{1} \partial \alpha} \\
\cdot & \frac{\partial^{2} l(\boldsymbol{\psi})}{\partial \lambda_{2}^{2}} & \cdots & \frac{\partial^{2} l(\boldsymbol{\psi})}{\partial \lambda_{2} \partial \lambda_{L}} & \frac{\partial^{2} l(\psi)}{\partial \lambda_{2} \partial \alpha} \\
\vdots & \vdots & \ddots & \vdots & \vdots \\
\cdot & \cdot & \ldots & \frac{\partial^{2} l(\boldsymbol{\psi})}{\partial \lambda_{L}^{2}} & \frac{\partial^{2} l(\boldsymbol{\psi})}{\partial \lambda_{L} \partial \alpha} \\
\cdot & . & \cdots & \cdot & \frac{\partial^{2} l(\boldsymbol{\psi})}{\partial \alpha^{2}}
\end{array}\right) .
$$

Os elementos de $\boldsymbol{B}(\boldsymbol{\psi})$ são dados por

$$
\begin{aligned}
\frac{\partial^{2} l(\boldsymbol{\psi})}{\partial \lambda_{\ell}^{2}} & =-(\alpha-1) \sum_{i=1}^{n} \frac{\nabla_{\ell}^{2}\left(t_{i}\right) e^{-u_{i}}}{\left(1-e^{-u_{i}}\right)^{2}}-n_{\ell} \lambda_{\ell}^{-2}, \quad \ell=1, \ldots, L, \\
\frac{\partial^{2} l(\boldsymbol{\psi})}{\partial \lambda_{\ell} \partial \lambda_{\ell^{*}}} & =-(\alpha-1) \sum_{i=1}^{n} \frac{\nabla_{\ell}\left(t_{i}\right) \nabla_{\ell^{*}}\left(t_{i}\right) e^{-u_{i}}}{\left(1-e^{-u_{i}}\right)^{2}}, \quad 1 \leq \ell<\ell^{*} \leq L, \\
\frac{\partial^{2} l(\boldsymbol{\psi})}{\partial \lambda_{\ell} \partial \alpha} & =\sum_{i=1}^{n} \frac{\nabla_{\ell}\left(t_{i}\right) e^{-u_{i}}}{1-e^{-u_{i}}}, \quad \ell=1, \ldots, L \\
e & \\
\frac{\partial^{2} l(\boldsymbol{\psi})}{\partial \alpha^{2}} & =-\frac{n}{\alpha^{2}} .
\end{aligned}
$$

\subsubsection{Função de log-verossimilhança com presença de censura à direita}

Por outro lado, na presença de censura à direita podemos adotar o seguinte esquema. Partimos do principio de que os tempos de censura e de falhas são independentes e são denotados por $Y_{i}$ e $C_{i}$, para $i=1, \ldots, n$ respectivamente. Os tempos observados são dados por $T_{i}=\min \left(Y_{i}, C_{i}\right)$ e o indicador de falha é denotado como $\delta_{i}=I\left(Y_{i} \leq C_{i}\right)$. Sejam $Y_{i}, C_{i}$ e $\delta_{i}$ os tempos de vida, censura e indicador de censuras como no capítulo anterior. A função de log-verossimilhança para $\boldsymbol{\psi}=(\boldsymbol{\lambda}, \alpha)$ (vide Lagakos [1979] para mais detalhes) é dada por 


$$
l(\boldsymbol{\psi})=\sum_{i=1}^{n}\left[\delta_{i} \log f\left(t_{i} ; \boldsymbol{\lambda}, \alpha, \boldsymbol{a}\right)+\left(1-\delta_{i}\right) \log S\left(t_{i} ; \boldsymbol{\lambda}, \alpha, \boldsymbol{a}\right)\right] .
$$

Para $\delta_{i}=1, i=1, \ldots, n$, a equação (5.13) é reduzida a (5.11). Finalmente, com base na equação (5.13) a inferência pode ser realizada de uma maneira similar tal como foi feito no caso censurado, como descrito acima.

O procedimento descrito nesta seção assume que o vector de partição $\boldsymbol{a}$ é conhecido. Na prática, tanto $L$, o número de intervalos na partição de $(0, \infty)$, e os valores exatos dos $a_{l}$ 's serão frequentemente desconhecidos e precisam ser sugeridos (ou estimados) a partir dos dados em questão. Uma abordagem razoável para este problema consiste em considerar no modelo $L=1,2, \ldots, L^{*}$ em que $L^{*}$ é um número relativamente pequeno, uma vez que um grande número de pontos de mudanças no modelo seria pouco provável de ser encontrado. Então, para cada valor de $L$, uma pesquisa sobre os valores alternativos de $a_{e l}$ 's pode ser implementado para identificar os limites da partição que maximizem a verossimilhança. Finalmente, pode-se comparar as verossimilhanças maximizadas para $L=1,2, \ldots, L^{*}$ para identificar o modelo preferido. A utilização de uma função de penalidade com base no número de parâmetros do modelo, tal como o critério de Akaike, será útil aqui, escolhendo sempre necessariamente o modelo mais apropriado correspondente ao caso $L=L^{*}$. Alguns esquemas de reamostragem, tal como, por exemplo, jackknife (Miller [1974]), permitirão determinar a variância das estimativas dos parâmetros neste cenário mais complexo. Uma abordagem alternativa, que é muito mais simples, consiste em traçar a função de risco acumulado empírica dos dados e, a partir de uma inspeção visual, identificar o número e localização dos possíveis pontos de mudança e, em seguida, usando esses valores como os valores conhecidos de $L$ e $\boldsymbol{a}$.

\subsection{Estudo de simulação}

Nesta seção apresentamos um estudo de simulação para o modelo apresentado na Seção 5.2. Assumimos $L=3$ e duas partições de tempo $a=(0.5,2)$ e $(2,6)$. Para cada partição, assumimos dois conjuntos de parâmetros, para $\boldsymbol{\lambda}=\left(\lambda_{1}, \lambda_{2}, \lambda_{3}\right), \boldsymbol{\lambda}=(1.1,0.8,0.5)$ e $\boldsymbol{\lambda}=(0.4,0.2,0.5)$, respectivamente. Para $\alpha$, assumimos dois valores: 0.8 e 1.2 (i.e., valores decrescentes e crescentes na função de risco em cada intervalo na partição de tempo). Para o tamanho da amostra, consideramos os casos $n=20, n=30, n=50$ e $n=100$. Para cada combinação de partição de tempo, $\lambda, \alpha$ e tamanho de amostra, simulamos 10.000 réplicas do modelo apresentado na Seção 5.2 e obtemos as estimativas de parâmetros usando máxima verossimilhança. Os resultados são apresentados na Tabela 5.1. Para cada caso, calculamos a média, o desvio padrão (DP) e a probabilidade de cobertura (PC, i.e., a proporção de vezes que o respectivo intervalo de confiança contém o verdadeiro valor do parâmetro). O último termo foi construído com base na distribuição assintótica para os estimadores de $\log \lambda_{i}, i=1,2,3$ e $\log \alpha$ (Sen et al. [2010], Teorema 8.3.1.). Observa-se que a estimativa de todos os parâmetros é aceitável. Como é esperado, o viés é reduzido quando $n$ aumenta. Finalmente, as PC's tornam-se mais próximas do valor nominal (95\%) quando $n$ aumenta. 


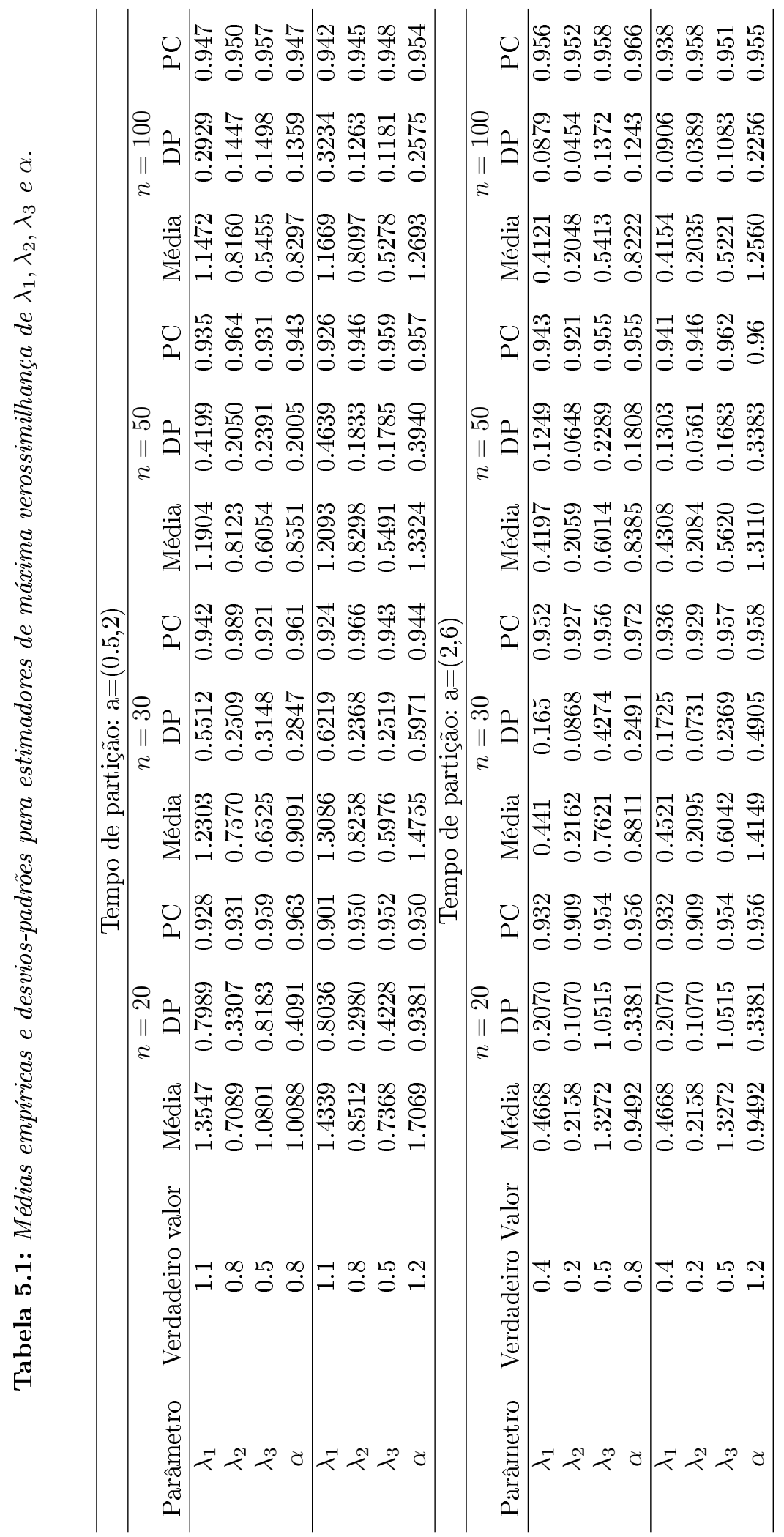




\subsection{Aplicações}

Nesta seção vamos ilustrar o uso do modelo ESP com dois conjuntos de dados reais, mostrando sua flexibilidade e aplicabilidade, quando comparado com outros modelos bem conhecidos na literatura.

O primeiro conjunto de dados é o tradicional conjunto de dados do Kevlar 49, que inclui 101 observações medidas completamente. Neste caso, nós comparamos a nossa proposta com outros modelos apresentados na literatura: a distribuição half-normal generalizada (HNG), proposta por Cooray e Ananda [2008], as extensões da distribuição exponencial propostas por Nadarajah e Haghighi [2011] (NH) e Gupta e Kundu [2001] (EG) e a distribuição kumaraswamy half-normal generalizada (Kw-HNG) proposta por Cordeiro et al. [2012].

O segundo conjunto de dados está relacionada com pacientes com câncer que receberam radioterapia. Os dados são caracterizados por algumas observações censuradas. Neste caso, nós comparamos o nosso modelo com outras abordagens para a modelagem de dados de tempo de vida, tais como a distribuição Weibull exponencializada de Mudholkar e Srivastava [1995] e a distribuição gamma exponencializada de Nadarajah e Kotz [2006b].

\subsubsection{Filamentos de Kevlar 49 /epoxy a 90\% de nível de estresse (exemplo sem cen- suras)}

Nesta subseção, apresentamos uma aplicação a um conjunto de dados reais relacionados com 101 observações que representam o tempo de resistência à ruptura de kevlar 49/epoxy que foram submetidos a pressão contínua constante no nível de estresse $90 \%$ (455.2 Ksi) e uma temperatura de $25^{\circ} \mathrm{C}$, até que todos tinham falhado, de modo que têm-se dados completos com tempos exatos de falha. Para estudos anteriores com este banco de dados veja (Andrews e Herzberg [1985] e Barlow et al. [1984]). O conjunto de dados pode ser encontrado no Apêndice A.1. Ressaltamos que este conjunto de dados não é o mesmo que o conjunto de dados do capítulo 3, mas tem as mesmas referências bibliográficas. A Figura 5.3 mostra a função de risco cumulativo com base na função de distribuição empírica para este conjunto de dados.

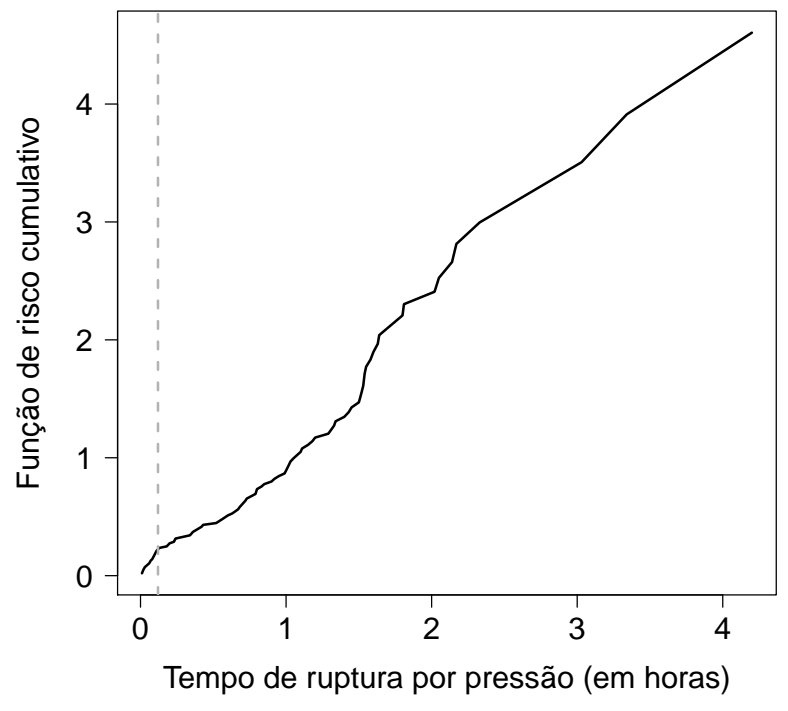

Figura 5.3: Função de risco cumulativo.

A Figura 5.3 sugere uma divisão dos tempos de vida em dois intervalos, isto é, $L=2$ e $a_{1}=0.12$. Além disso, comparamos nosso modelo ESP com outros modelos considerados na literatura como mencionado acima. A parametrização dos modelos pode ser vista na Tabela 2.3. A Tabela 5.2 mostra as estimativas para todos os modelos e o critério de Akaike (AIC, Akaike, H. [1974]). Am- 
bos modelos ES $(L=2)$ e o $\operatorname{ESP}(L=2)$ ajustam melhor os dados do que os modelos que não envolvem ponto de mudança, mas o modelo $\operatorname{ESP}(L=2)$ é selecionado segundo o critério de Akaike.

Tabela 5.2: As estimativas dos parâmetros (com (DP) indicando o desvio padrão) e as distribuições ajustadas para o conjunto de dados completos de kevlar.

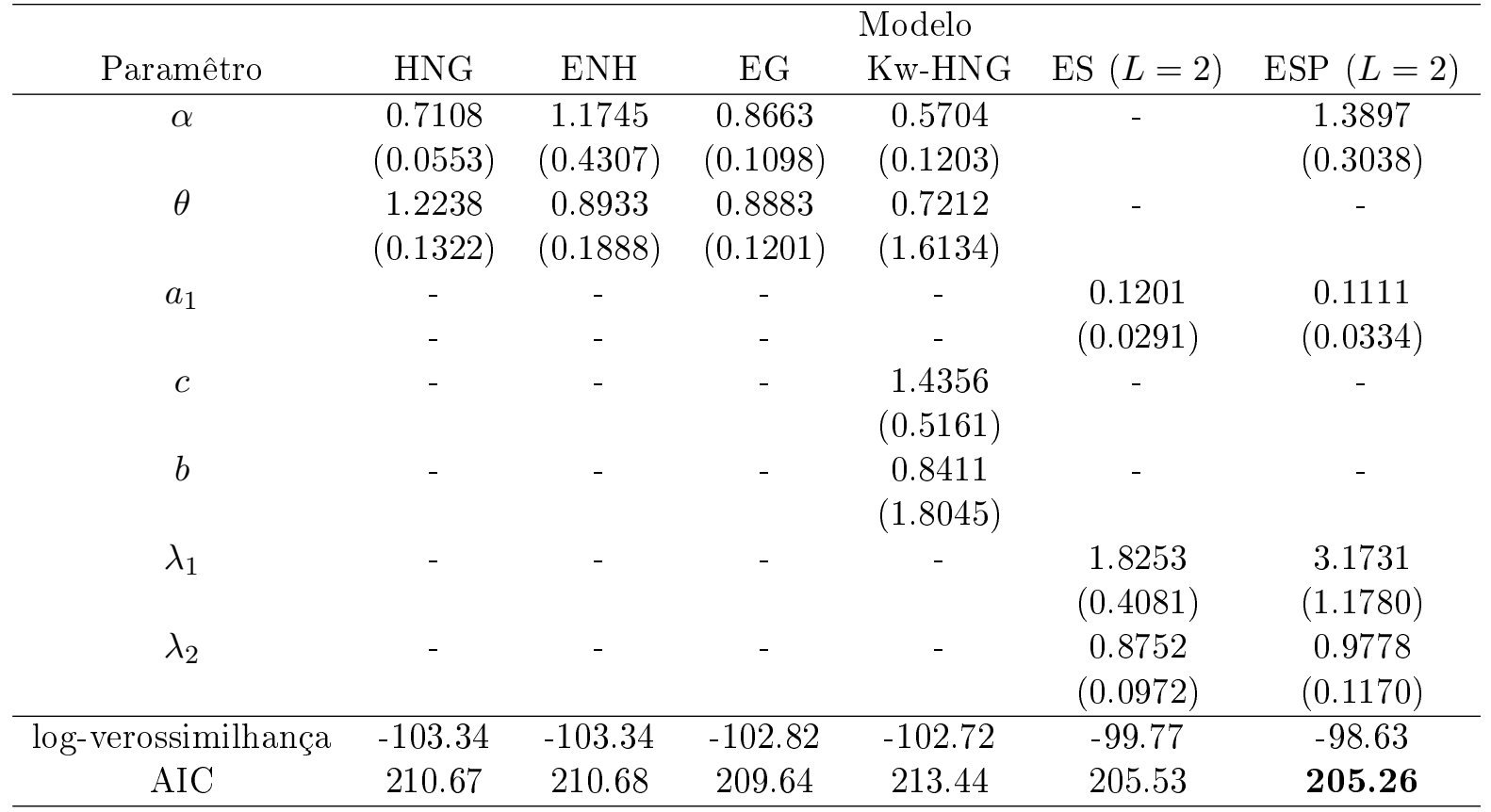

Para este conjunto de dados, Cooray e Ananda [2008] discutiram a possibilidade de que o dado maior registrado (7.89) pode ser discrepante, o que pode estar influenciando as estimativas. Por esta razão, ajustamos os mesmos modelos, mas sem o dado maior. Na Tabela 5.3 mostramos os resultados. Mais uma vez, o melhor ajuste é fornecido pelo modelo ESP com $L=2$ em comparação com os outros modelos em questão. Notamos que, em contraste com os resultados de Cooray e Ananda [2008], obtém-se um melhor encaixe para o modelo ESP com ou sem o ponto final dos dados, que mostra a flexibilidade do nosso modelo para os dados ajustados, com alta ou baixa curtose. Note-se que o teste da razão de versomilhanças pode ser utilizado para testar a hipótese $H_{0}: \alpha=1$ versus $H_{1}: \alpha \neq 1$ para decidir entre o modelo ES e ESP. Com o conjunto de dados completo, o valor-p do teste é de 0.131 , mas sem a última observação, o valor-p é reduzido para 0.065 . Em ambos os casos, a preferência para o modelo de ESP pode ser marginalmente fundamentada. Finalmente, a evidência visual de que o ajuste do modelo ESP é melhor é fornecida pela Figura 5.4. 
Tabela 5.3: As estimativas dos parâmetros (com (DP), indicando desvio padrão) e distribuições ajustadas (dados kevlar sem observação 101).

\begin{tabular}{|c|c|c|c|c|c|c|}
\hline Paramêtro & HNG & ENH & EG & $\begin{array}{l}\text { Modelo } \\
\text { Kw-HNG }\end{array}$ & $\mathrm{ES}(L=2)$ & $\operatorname{ESP}(L=2)$ \\
\hline \multirow[t]{2}{*}{$\alpha$} & 0.7824 & 1.2543 & 0.9119 & 0.7834 & - & 1.4779 \\
\hline & $(0.0645)$ & $(0.3778)$ & $(0.1162)$ & $(0.2472)$ & & $(0.3167)$ \\
\hline$\theta$ & $\begin{array}{c}1.1572 \\
(0.1133)\end{array}$ & $\begin{array}{c}0.7408 \\
(0.3375)\end{array}$ & $\begin{array}{c}0.9857 \\
(0.1309)\end{array}$ & $\begin{array}{c}0.8537 \\
(1.3619)\end{array}$ & - & - \\
\hline \multirow[t]{2}{*}{$a_{1}$} & - & - & - & - & 0.1113 & 0.1113 \\
\hline & - & - & - & - & $(0.0291)$ & $(0.0155)$ \\
\hline$c$ & - & - & - & $\begin{array}{c}0.9231 \\
(0.3079)\end{array}$ & - & - \\
\hline$b$ & - & - & - & $\begin{array}{c}0.6820 \\
(1.0704)\end{array}$ & - & - \\
\hline$\lambda_{1}$ & - & - & - & - & $\begin{array}{c}1.8761 \\
(0.4304)\end{array}$ & $\begin{array}{c}3.4581 \\
(1.2048)\end{array}$ \\
\hline$\lambda_{2}$ & - & - & - & - & $\begin{array}{c}0.9474 \\
(0.1053)\end{array}$ & $\begin{array}{c}1.0704 \\
(0.1251)\end{array}$ \\
\hline log-verossimilhança & -93.84 & -95.21 & -95.26 & -93.77 & -92.42 & -90.72 \\
\hline $\mathrm{AIC}$ & 191.67 & 194.41 & 194.51 & 195.53 & 190.84 & 189.44 \\
\hline
\end{tabular}
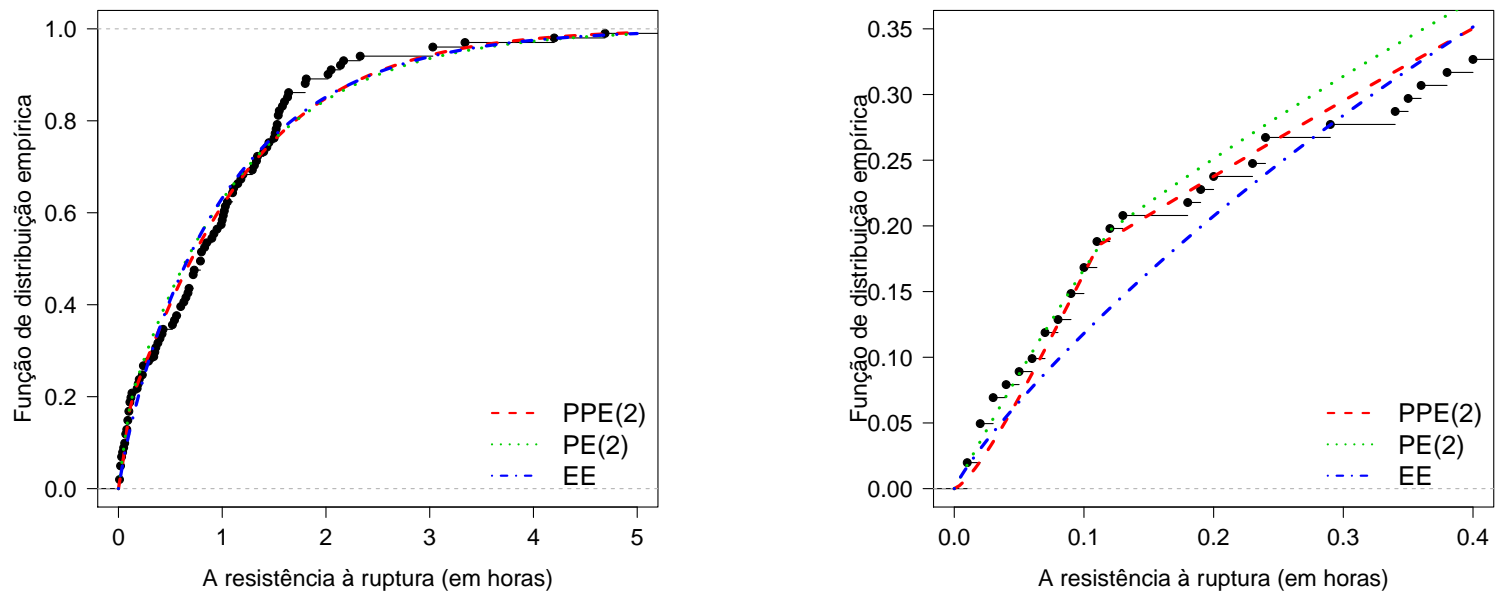

Figura 5.4: Função de distribuição empírica e funções de distribuição cumulativa estimada para alguns modelos do conjunto de dados kevlar 49/epoxy (sem o ponto final dos dados). O painel à direita é um destaque.

\subsubsection{Estudo de radioterapia (dados censurados)}

Este conjunto de dados está relacionada a pacientes com determinado tipo de câncer que receberam radioterapia. O tempo (em dias) foi medido a partir do momento em que o paciente recebeu o tratamento até a morte do paciente ou o último tempo de controle para o qual temos informações. O conjunto de dados completos [Louzada-Neto et al., 2001] é mostrado abaixo, em que ${ }^{+}$indica os pacientes com informações incompletas (censurados).

$7,34,42,63,64,74^{+}, 83,84,91,108,112,129,133,133,139,140,140,146,149,154,157,160,160,165$, $173,176,185^{+}, 218,225,241,248,273,277,279^{+}, 297,319^{+}, 405,417,420,440,523^{+}, 523,583,594,1101$, 
Tabela 5.4: Critério de AIC para o conjunto de dados de radioterapia.

\begin{tabular}{ccccc}
\hline & WE & GE & $\mathrm{ES}(2)$ & $\mathrm{ESP}(2)$ \\
\hline log-verossimilhança & -45.27 & -48.30 & -44.67 & -41.93 \\
AIC & 96.53 & 102.59 & 95.33 & $\mathbf{9 1 . 8 6}$ \\
\hline
\end{tabular}

$1116^{+}, 1146,1226^{+}, 1349^{+}, 1412^{+}, 1417$.

A principal característica deste conjunto de dados é a ausência de tempos observados (falha ou censurados) entre os días 594 e 1101 (um período de 507 dias, mais de um ano). Esta é uma característica que faz termos dificuldade na obtenção de um bom ajuste para qualquer modelo que não seja segmentado. Claro que, com uma definição apropriada para a partição dos tempos de vida, o modelo ESP pode adaptar-se a este problema.

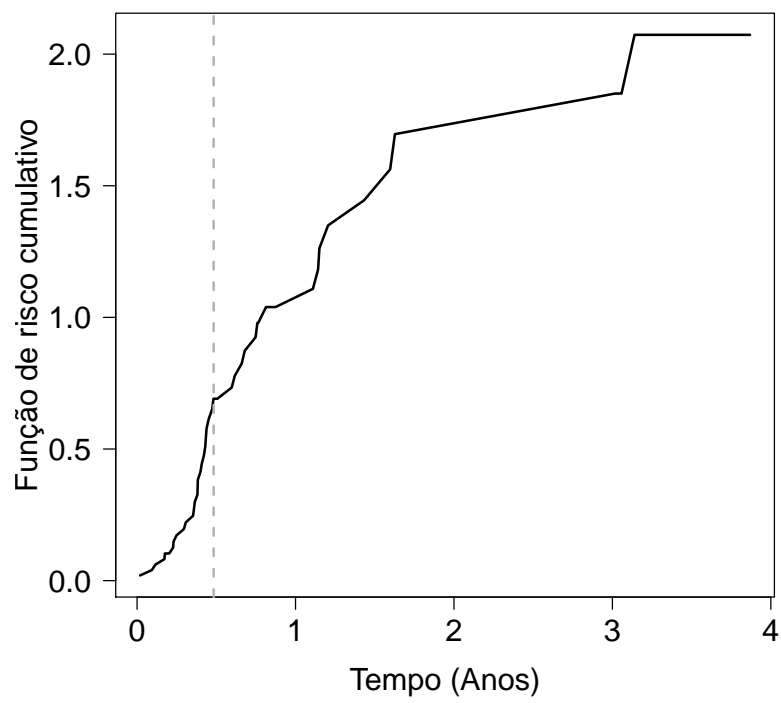

Figura 5.5: Função de risco cumulativo estimado para dados do estudo de radioterapia.

A Figura 5.5 mostra a função de risco acumulado estimado para o conjunto de dados de radioterapia com base na estimativa de Kaplan-Meier. A partir desta figura, pode-se sugerir uma partição em dois ou três intervalos. O modelo ESP com $L=2$ intervalos de tempo foi comparado com o modelo Weibull exponencializado (Mudholkar e Srivastava [1995]) e o modelo Gamma Exponencializado (Nadarajah e Kotz [2006b]). A inferência dos modelos foi baseada na função de verossimilhança para dados censurados de uma forma semelhante a expressão (5.13). As funções de densidade e de sobrevivência para esses modelos são apresentados na Tabela 2.2. Note que ambos modelos têm três parâmetros. Na Tabela 5.4 mostramos o valor do critério AIC para os três modelos, sugerindo a seleção do modelo ESP com 2 intervalos de tempo. O valor- $p$ para o teste da razão de verossimilhanças da hipótese $H_{0}: \alpha=1$ versus $H_{1}: \alpha \neq 1$ é 0.019 , com um nível de significância $<5 \%$, indicando que o modelo ESP é melhor para este conjunto de dados em relação ao modelo ES. Estimativas para este modelo são apresentada na Tabela 5.5. Finalmente, a Figura 5.6 ilustra o melhor desempenho do modelo ESP entre os outros modelos considerados. 
Tabela 5.5: Parâmetros estimados para o modelo ESP.

\begin{tabular}{ccc}
\hline Paramêtro & Estimativas & DP \\
\hline$\lambda_{1}$ & 2.7238 & 0.4157 \\
$\lambda_{2}$ & 0.6434 & 0.0223 \\
$\alpha$ & 2.4153 & 0.4588 \\
$a_{1}$ & 0.4832 & 0.0295 \\
\hline
\end{tabular}

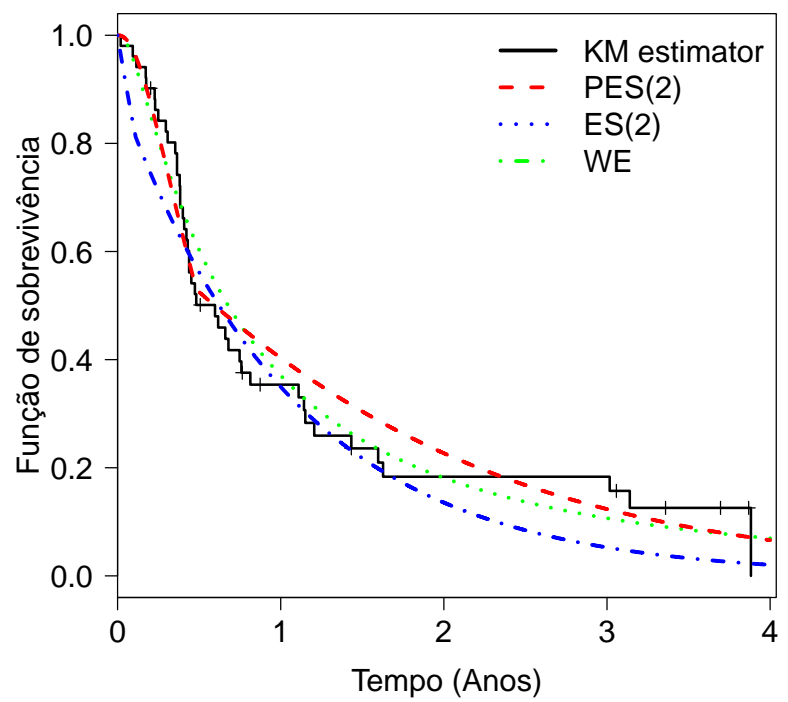

Figura 5.6: Função de sobrevivência estimado para os dados do estudo de radioterapia sob o modelo ESP(2), $E S(2)$ e WE.
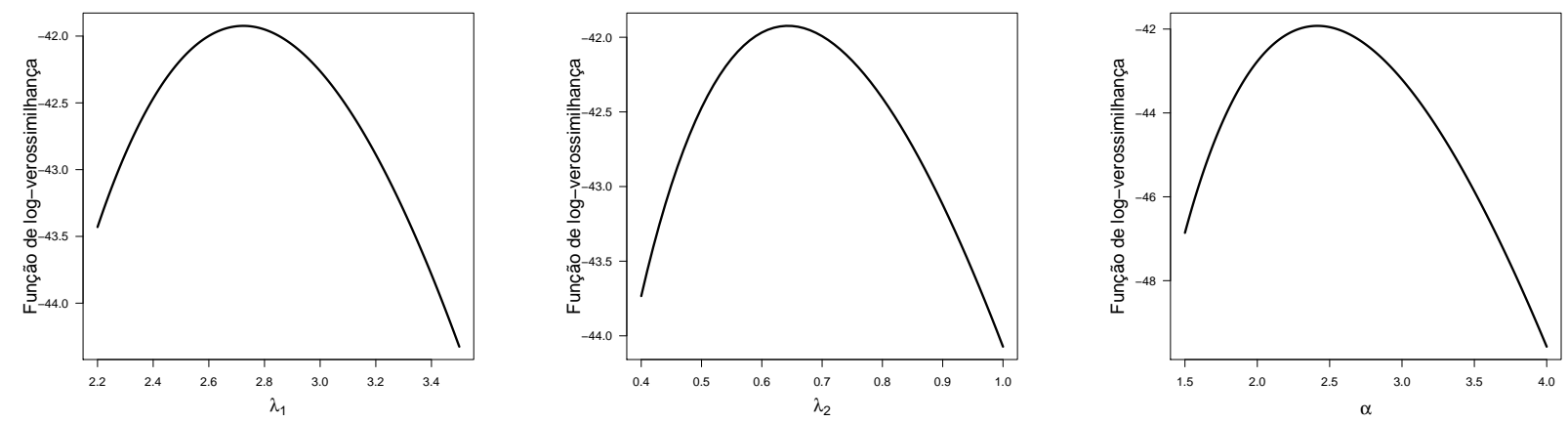

Figura 5.7: Perfil de log-verossimilhança para $\lambda_{1}, \lambda_{2}$ e $\alpha$ no modelo ESP com observações censuradas no conjunto de dados do estudo de radioterapia.

\subsection{Observações finais}

Neste capítulo uma nova distribuição segmentada, mais flexivel, que a exponencial (que é um caso particular), é apresentada, denominada distribuição ESP. Ela acomoda diferentes formas não constantes para a função nos diferentes intervalos de tempo. O modelo depende de uma partição de tempo que pode ser tomada como sendo conhecida, ou pode ser estimada a partir dos dados. Estudos de simulação sugerem que os parâmetros podem ser estimados de uma forma aceitável para diferentes tamanhos de amostra com base no método de máxima verosimilhança. Na aplicação, o critério de AIC foi utilizado para seleção de modelos. O modelo ESP foi ajustado com outros 
modelos propostos recentemente na literatura para o conhecido conjunto de dados Kevlar 49/epoxy e os dados de radioterapia para um certo tipo de câncer, caracterizado pela presença de muitas observações censuradas. Em ambos os casos, o desempenho do modelo ESP foi o melhor entre os modelos considerados. 


\section{Capítulo 6}

\section{Distribuição half-normal potência}

Neste capítulo consideramos uma extensão da distribuição half-normal com base na distribuição do máximo de uma amostra aleatória. Mostra-se que esta distribuição pertence à familia das distribuiçôes beta generalizada half-normal. Propriedades de sua densidade são investigadas, estimação por máxima verossimilhança é implementada e a matriz de informação de Fisher é derivada. Uma ilustração com dados reais é apresentada e as comparaçôes com extensões alternativas da distribuição half-normal revelam o bom desempenho do modelo.

\subsection{Motivação}

A distribuição half-normal é um modelo que tem sido muito utilizado para o modelagem de dados na área de sobrevivência e confiabilidade. A função de densidade pode ser encontrada na Tabela 2.2 e na expressão (2.15).

Este capítulo concentramos em estudar a distribuição que é gerada quando consideramos a fda do modelo half-normal na equação (5.2). A distribuição half-normal introduzida é chamada de distribuição half-normal potência (HNP). A família HNP é uma subfamília das distribuições consideradas em Pescim et al. [2010]. A distribuição HNP tem apenas dois parâmetros e pode ser usada para ajuste de dados positivos de confiabilidade ou de sobrevivência, sendo assim uma alternativa às distribuições half-normal, gama e Weibull, entre outras. Uma vantagem do modelo PHN é que ele pode produzir funções de risco na forma de banheira, o que não acontece com os modelos de Weibull e half-normal.

\subsection{Distribuição half-normal potência}

Definição 6.2.1 Uma variável aleatória T segue uma distribuição half-normal potência com parâmetro de escala $\sigma$ e parâmetro de forma $\alpha$ se sua fdp é dada por

$$
f_{T}(t ; \sigma, \alpha)=\frac{2 \alpha}{\sigma} \phi\left(\frac{t}{\sigma}\right)\left(2 \Phi\left(\frac{t}{\sigma}\right)-1\right)^{\alpha-1},
$$

em que $\sigma>0, \alpha>0, t>0$. Usamos a notação $T \sim H N P(\sigma, \alpha)$.

Observação 9 Note que para $\alpha=1$, obtemos o modelo half-normal com parâmetro de escala $\sigma$.

A Figura 6.1 representa graficamente a função de densidade para o modelo HNP para alguns valores dos parâmetros $\sigma$ e $\alpha$.

\subsubsection{Principais propriedades}

A seguir, as funções de distribuição acumulada, de sobrevivência, de risco e de quantis são derivadas para o modelo HNP.

Definição 6.2.2 A função de distribução para $T \sim H N P(\sigma, \alpha)$ é dada por

$$
F(t ; \sigma, \alpha)=\left(2 \Phi\left(\frac{t}{\sigma}\right)-1\right)^{\alpha}
$$

em que $\sigma>0, \alpha>0$ e $t>0$. 

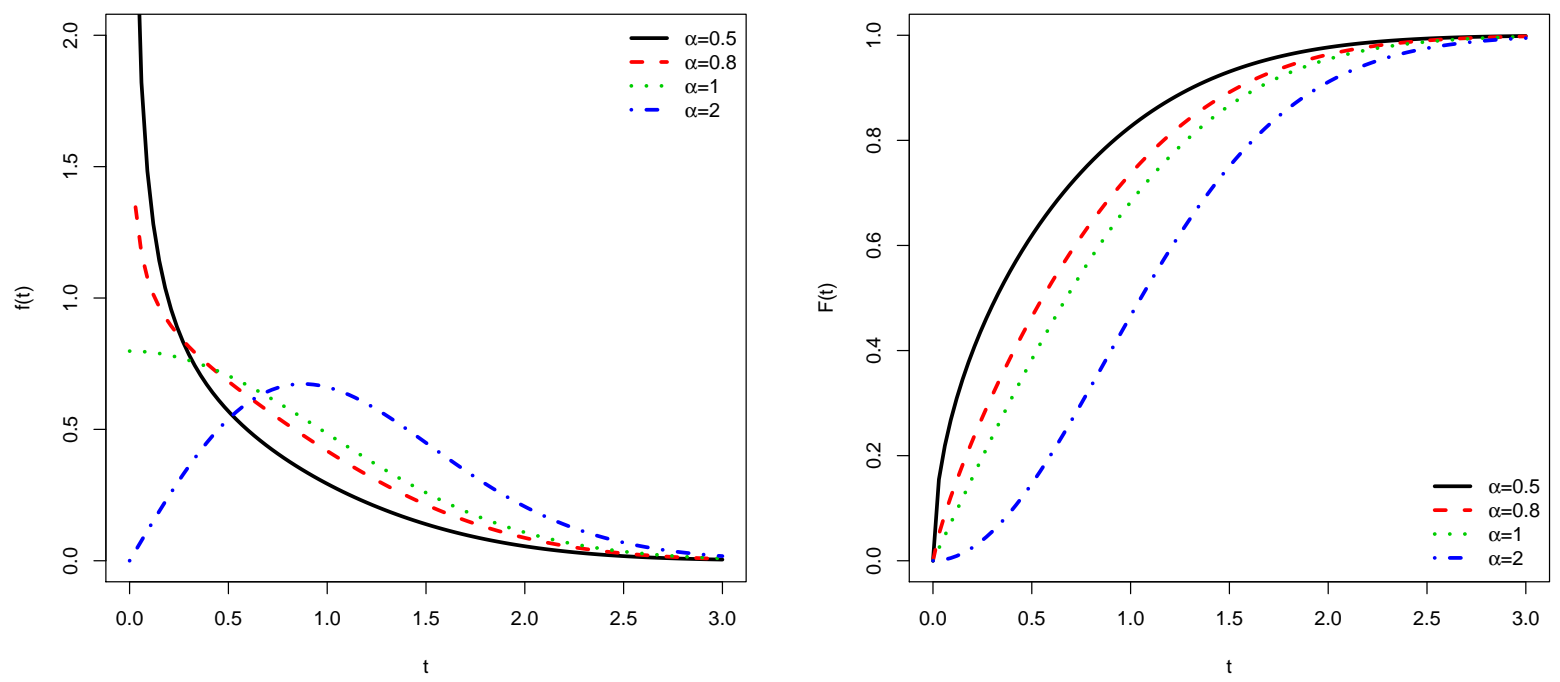

Figura 6.1: Função de densidade de $T$ para $\sigma=1, \alpha=0.5,0.8,1,1.2,2$. (esquerda) e função de distribução para $\sigma=1$ e $\alpha=0.5,1,1.2,4,10$.(direita) do modelo PHN.

As correspondentes funções de sobrevivência e de risco são, respectivamente, dadas por

$$
S(t ; \sigma, \alpha)=1-F_{T}(t)=1-\left(2 \Phi\left(\frac{t}{\sigma}\right)-1\right)^{\alpha}
$$

$\mathrm{e}$

$$
h(t ; \sigma, \alpha)=\frac{f(t)}{S(t)}=\frac{\frac{2 \alpha}{\sigma} \phi\left(\frac{z}{\sigma}\right)\left(2 \Phi\left(\frac{t}{\sigma}\right)-1\right)^{\alpha-1}}{1-\left(2 \Phi\left(\frac{t}{\sigma}\right)-1\right)^{\alpha}} .
$$

A Figura 6.1 mostra os gráficos da função de distribuição e a função de sobrevivência para alguns valores de parâmetros $\sigma$ e $\alpha$. A Figura 6.2 mostra o gráfico da função de sobrevivência e da função de risco para a distribuição potência half-normal. Observe que há casos em que a função de risco é inicialmente decrescente e, em seguida, vai crescendo sendo assim da forma de banheira.

Proposição 6.2.1 Seja $T \sim H N P(\sigma, \alpha)$. Temos que a função de quantis é dada por

$$
Q(p)=\sigma \Phi^{-1}\left(\frac{1+p^{1 / \alpha}}{2}\right), \quad 0<p<1 .
$$

Assim, temos que

1. primeiro quartil $=\sigma \Phi^{-1}\left(\frac{1+4^{1 / \alpha}}{2^{2 / \alpha+1}}\right)$

2. $\operatorname{Mediana}(T)=\sigma \Phi^{-1}\left(\frac{1+2^{1 / \alpha}}{2^{1 / \alpha+1}}\right)$

3. terceiro quartil $=\sigma \Phi^{-1}\left(\frac{3+4^{1 / \alpha}}{2^{2 / \alpha+1}}\right)$.

Observação 10 Note que as funções $F_{T}(t ; \sigma, \alpha), Q(p ; \sigma, \alpha), S(t ; \sigma, \alpha)$ e $h(t ; \sigma, \alpha)$ dependem apenas de $\Phi(\cdot)$, que está implementada na linguagem $R$ Core Team [2015] como a função pnorm.

\subsubsection{Caracterizações da distribuição PHN}

Na concepção de um modelo estocástico para um problema de modelagem estatística, se o modelo obedece a certas características de interesse. Para este fim, o investigador irá invocar as 

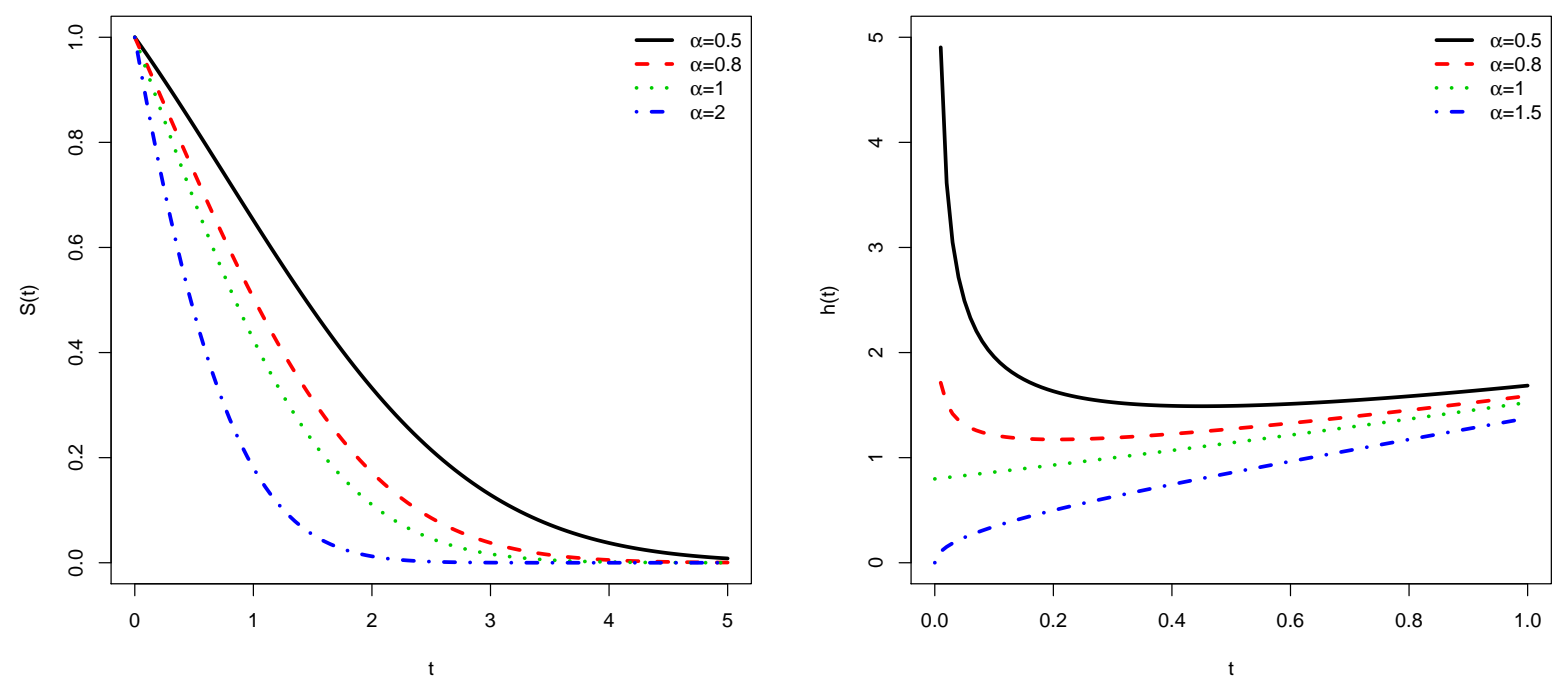

Figura 6.2: Função de sobrevivência $T$ para $\sigma=1$ e $\alpha=0.5,0.8,1,1.2,2$.(direita) e função de risco para $\sigma=1$ e $\alpha=0.5,0.8,1,1.2,2$.

caracterizações da distribuição selecionada. De um modo geral, o problema de caracterização de uma distribuição é um problema importante em vários campos e tem atraído recentemente a atenção de muitos investigadores. Consequentemente, vários resultados de caracterizações foram relatados na literatura. Essas caracterizações foram estabelecidas em muitas direções diferentes. Nesta seção, apresentamos ainda mais caracterizações da distribuição PHN. Estas caracterizações são baseadas na esperança condicional de uma função da variável aleatória. Aqui, nós empregamos uma única função $\psi$ de $T$ e os resultados da caracterização em termos de $\psi(T)$.

Proposição 6.2.2 Seja $T: \Omega \rightarrow(a, b)$ uma variável aleatória contínua com $f d a \quad F$. Seja $\psi(T)$ uma função diferenciável em $(a, b)$ com $\lim _{T \rightarrow b^{-}} \psi(t)=1$. Em seguida, para $\delta \neq 1$,

$$
E[\psi(T) \mid T \leq t]=\delta \psi(t), \quad t \in(a, b),
$$

se, e somente se,

$$
\psi(t)=(F(t))^{\frac{1}{\delta}-1}, \quad t \in(a, b)
$$

Prova É direta.

A forma mais geral da equação (6.2) é dada abaixo.

Proposição 6.2.3 Seja $T: \Omega \rightarrow(a, b)$ uma variável aleatória contínua com $f d a \quad F$. Seja $\psi(t)$ uma função diferenciável em $(a, b)$ com $\lim _{t \rightarrow a^{+}} \psi(t)=\delta>1$ e $\lim _{t \rightarrow b^{-}} \psi(t)=\infty$. Então,

$$
E\left[(\psi(T))^{\delta} \mid T \leq t\right]=\delta(\psi(t))^{\delta-1}, \quad t \in(a, b),
$$

se, e somente se,

$$
\psi(t)=\delta\left[1+(F(t))^{\frac{1}{\delta-1}}\right]^{-1}, \quad t \in(a, b) .
$$


Prova A partir da equação (6.3), temos que

$$
\int_{a}^{t}(\psi(u))^{\delta} f(u) d u=\delta(\psi(t))^{\delta-1} F(t)
$$

Tomando as derivadas de ambos lados da equação acima e reorganizando os termos, chegamos a

$$
\frac{f(t)}{F(t)}=(\delta-1)\left\{-\frac{\psi^{\prime}(t)}{\psi(t)}+\frac{\psi^{\prime}(t)}{\psi(t)-\delta}\right\}
$$

Integrando ambos lados desta equação de $t$ a $b$ e usando a condição $\lim _{t \rightarrow b^{-}} \psi(t)=\infty$, nós obtemos a expressão (6.4).

Observação 11 (a) Tomando, por exemplo, $(a, b)=(0, \infty)$ e $\psi(t)=\left(2 \Phi\left(\frac{t}{\sigma}\right)-1\right)^{\frac{\alpha(1-\delta)}{\delta}}$, a Proposição 6.2.2 dá uma caracterização da distribuição de PHN. (b) Tomando, por exemplo, $(a, b)=(0, \infty)$ e $\psi(t)=\frac{\delta}{1+\left(2 \Phi\left(\frac{t}{\sigma}\right)-1\right)^{\frac{\alpha}{\delta-1}}}$, a Proposição 6.2.3 dá uma caracterização da distribuição PHN.

\subsubsection{Momentos}

Os momentos do modelo PHN podem ser calculados numericamente com a rotina "integrate" do software R (R Core Team [2015]). A seguinte proposição apresenta o $r$-ésimo momento de uma variável aleatória seguindo a distribuição PHN.

Proposição 6.2.4 O r-ésimo momento da variável aleatória $T \sim H N P(\sigma, \alpha)$ é dado por

$$
\mu_{r}=E\left(T^{r}\right)=\alpha \sigma^{r} \xi_{r}(\alpha), \quad r=1,2, \ldots,
$$

em que $\xi_{r}(\alpha)=\int_{0}^{1}\left(\Phi^{-1}\left(\frac{1+u}{2}\right)\right)^{r} u^{\alpha-1} d u$, que podem ser calculados numericamente.

Prova A definição do momento implica

$$
\mu_{r}=E\left(T^{r}\right)=\int_{0}^{\infty} \frac{2 \alpha}{\sigma} t^{r} \phi\left(\frac{t}{\sigma}\right)\left(2 \Phi\left(\frac{t}{\sigma}\right)-1\right)^{\alpha-1} d t
$$

O resultado segue depois de fazer a mudança de variável $u=2 \Phi\left(\frac{t}{\sigma}\right)-1$.

Corolário 6.2.1 Por conseguinte, os quatro primeiros momentos e a variância são dadas por

1. $\mu_{1}=E(T)=\alpha \sigma \xi_{1}(\alpha)$,

2. $\mu_{2}=E\left(T^{2}\right)=\alpha \sigma^{2} \xi_{2}(\alpha)$,

3. $\mu_{3}=E\left(T^{3}\right)=\alpha \sigma^{3} \xi_{3}(\alpha)$,

4. $\mu_{4}=E\left(T^{4}\right)=\alpha \sigma^{4} \xi_{4}(\alpha)$

$e$

5. $\operatorname{Var}(T)=\alpha \sigma^{2}\left[\xi_{2}(\alpha)-\alpha \xi_{1}^{2}(\alpha)\right]$. 
Corolário 6.2.2 Os coeficientes de assimetria e curtose são dados, respectivamente, pelas expressões

$e$

$$
\sqrt{\beta_{1}}=\frac{\kappa_{3}(\alpha)-3 \alpha \xi_{1}(\alpha) \xi_{2}(\alpha)+2 \alpha^{2} \xi_{1}(\alpha)^{3}}{\sqrt{\alpha}\left(\xi_{2}(\alpha)-\alpha \xi_{1}(\alpha)^{2}\right)^{3 / 2}}
$$

$$
\beta_{2}=\frac{\xi_{4}(\alpha)-4 \alpha \xi_{1}(\alpha) \xi_{3}(\alpha)+6 \alpha^{2} \xi_{1}(\alpha)^{2} \xi_{2}(\alpha)-3 \alpha^{3} \xi_{1}(\alpha)^{4}}{\alpha\left(\xi_{2}(\alpha)-\alpha \xi_{1}(\alpha)^{2}\right)^{2}} .
$$

Na Figura 6.3 apresentamos a esperança e a variância em função dos parâmetros $\alpha$ e $\sigma$. Também apresentamos os coeficientes de assimetria e de curtose para alguns valores do parâmetro $\alpha$. Note que o caso $\alpha=1$ coincide com o coeficiente de assimetria e curtose do modelo half-normal padrão(linha tracejada), que são dados por

$$
\sqrt{\beta_{1}}=\frac{\sqrt{2}(4-\pi)}{(\pi-2)^{3 / 2}}=0.995 \quad \text { e } \quad \beta_{2}=\frac{\pi(3 \pi-4)-12}{(\pi-2)^{2}}=3.869, \quad \text { respectivamente. }
$$

\subsubsection{Entropia de Shannon}

A entropia de uma variável aleatória T é uma medida de sua incerteza. A medida de entropia de Shannon é definida por

$$
J_{S}=-E\left(\log \left(f_{T}(t)\right)\right) .
$$

Verifica-se, depois de algumas manipulações algébricas que a entropia de Shannon para o modelo HNP é:

$$
J_{S}=-\log (2)-\log (\alpha)+\log (\sigma)+\log (\sqrt{2 \pi})+\frac{\alpha \kappa_{2}(\alpha)}{2}+\frac{\alpha-1}{\alpha},
$$

com $\kappa_{2}(\alpha)$ definido na Proposição (6.2.4).

Observação 12 Note-se que quando $\alpha=1$ (que é a distribuição half-normal), temos que $\kappa_{2}(\alpha)=$ $\alpha^{-1}$. Em seguida, obtém-se, como um caso particular, a entropia de Shannon da distribuição halfnormal (vide Ahsanullah et al. [2014]), que é dada por

$$
J_{S}=\frac{1}{2}-\log \left(\sqrt{\frac{2}{\pi}} \frac{1}{\sigma}\right)
$$

\subsection{Inferência}

Nesta seção, serão discutidos os estimadores de momentos, e de máxima verosimilhança, assim como a matriz de informação de Fisher do modelo HNP.

\subsubsection{Estimadores de momentos}

Utilizando os dois primeiros momentos, as equações de momento no modelo HNP são dadas por

$$
\bar{T}=\alpha \sigma \kappa_{1}(\alpha) .
$$

e

$$
\overline{T^{2}}=\alpha \sigma^{2} \kappa_{2}(\alpha) .
$$

Resolvendo para $\sigma$ na equação (6.6), segue que

$$
\sigma=\frac{\bar{T}}{\alpha \kappa_{1}(\alpha)} .
$$

Assim, substituindo $\sigma$ dado na equação (6.8), em (6.7), segue que

$$
\overline{T^{2}} \alpha \kappa_{1}(\alpha)^{2}-\bar{T}^{2} \kappa_{2}(\alpha)=0 .
$$



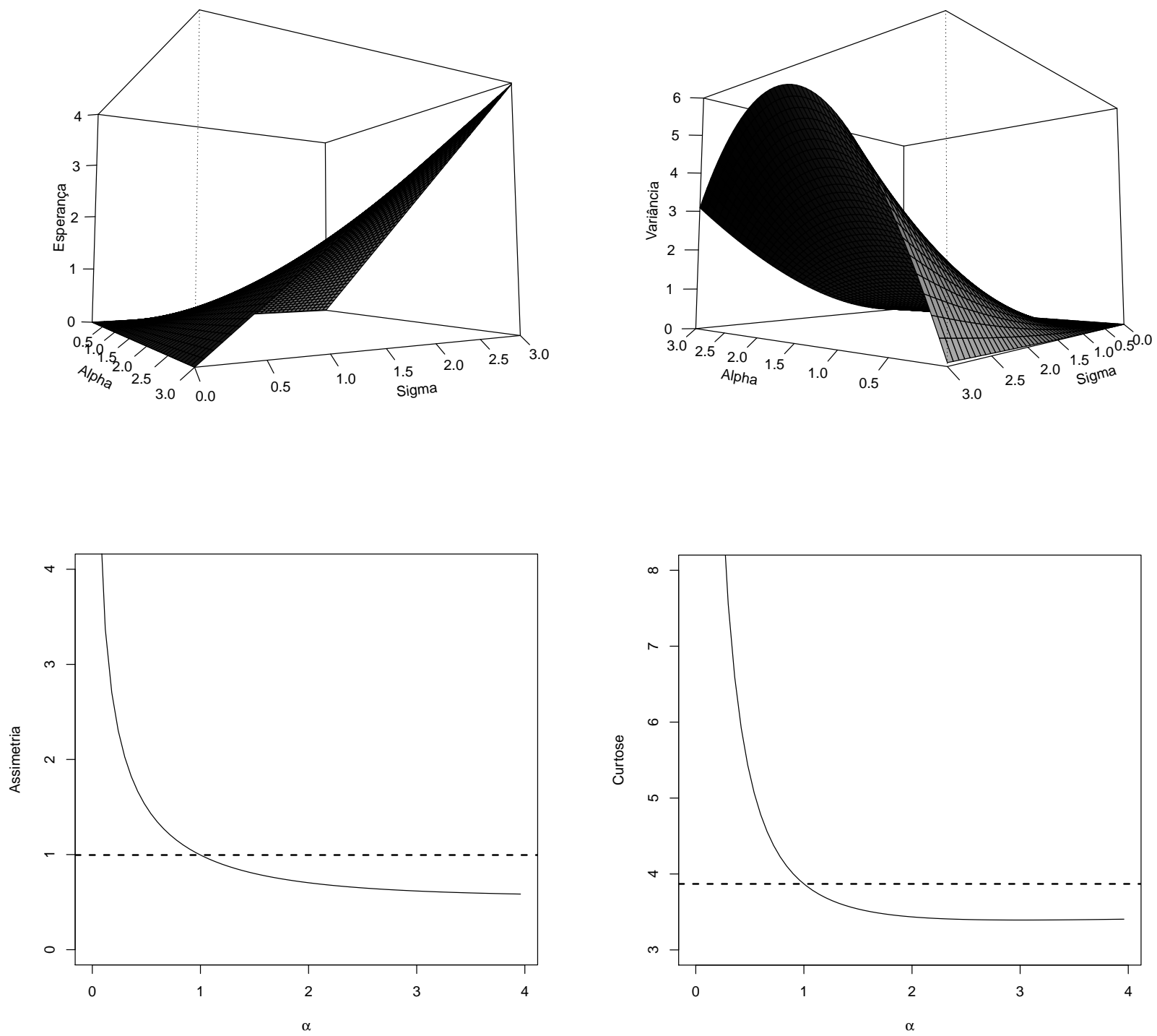

Figura 6.3: Algumas características da distribuição PHN para um conjunto diferente de parâmetros. Painel superior esquerdo: média. Painel superior direito: variância. Painel inferior esquerdo: coeficiente de assimetria. Painel inferior direito: coeficiente de curtose.

Resolvendo a equação dada em (6.7) para $\alpha$ obtemos $\widehat{\alpha}_{M}$, e, portanto, a substituição $\alpha$ por $\widehat{\alpha}_{M}$ na equação (6.8) obtemos $\widehat{\sigma}_{M}$. Isto conduz a estimadores momentos $\left(\widehat{\sigma}_{M}, \widehat{\alpha}_{M}\right)$ para $(\sigma, \alpha)$. A equação dada em (6.9), pode ser resolvida numericamente usando a função solve disponível em software MAPLE.

\subsubsection{A função de log-verosimilhança}

Seja $t_{1}, \ldots, t_{n}$ observações de uma amostra aleátoria da variável aleátoria $T \sim \operatorname{PHN}(\sigma, \alpha)$. A função de verossimilhança para $\theta=(\sigma, \alpha)$ é dada por $\sum_{i=1}^{n} l\left(\theta ; t_{i}\right)$, em que $l(\theta ; t)$ a função de verossimilhança para $\theta$ dado $T$, que fica dada por

$$
l(\theta ; t)=\log (2)+\log (\alpha)-\log (\sigma)-\log (\sqrt{2 \pi})-\frac{t^{2}}{2 \sigma^{2}}+(\alpha-1) \log \left(2 \Phi\left(\frac{t}{\sigma}\right)-1\right) .
$$




\subsubsection{Função escore}

Depois de algumas manipulações algébricas, obtemos que a função de score é $\sum_{i=1}^{n} S_{\theta}\left(\theta ; t_{i}\right)$, em que $S_{\theta}(\theta, t)=\partial l(\theta, t) / \partial \theta$ é o vetor $\left(S_{\sigma}, S_{\alpha}\right)$ com elementos

$$
S_{\sigma}=-\frac{1}{\sigma}+\frac{t^{2}}{\sigma^{3}}-\frac{2(\alpha-1)}{\sigma^{2}}\left(\frac{t \phi\left(\frac{t}{\sigma}\right)}{2 \Phi\left(\frac{t}{\sigma}\right)-1}\right) \quad e \quad S_{\alpha}=\frac{1}{\alpha}+\log \left[2 \Phi\left(\frac{t}{\sigma}\right)-1\right] .
$$

As segundas derivadas de $l(\theta ; t)$ são

$$
\begin{aligned}
& \frac{\partial^{2} l(\theta ; t)}{\partial \sigma^{2}}=\frac{1}{\sigma^{2}}-\frac{3 t^{2}}{\sigma^{4}}+\frac{2(\alpha-1) t \phi\left(\frac{t}{\sigma}\right)}{\sigma^{3}\left[2 \Phi\left(\frac{t}{\sigma}\right)-1\right]}\left(2-\frac{t^{2}}{\sigma^{2}}-\frac{2 t \phi\left(\frac{t}{\sigma}\right)}{\sigma\left(2 \Phi\left(\frac{t}{\sigma}\right)-1\right)}\right), \\
& \frac{\partial^{2} l(\theta ; t)}{\partial \alpha^{2}}=-\frac{1}{\alpha^{2}} \quad e \frac{\partial^{2} l(\theta ; t)}{\partial \sigma \partial \alpha}=-\frac{2 t \phi\left(\frac{t}{\sigma}\right)}{\sigma^{2}\left(2 \Phi\left(\frac{t}{\sigma}\right)-1\right)} .
\end{aligned}
$$

\subsubsection{Matriz de informação de Fisher}

Usando as segundas derivadas acima, a matriz de informação de Fisher para a distribuição HNP pode ser escrito como

$$
I_{F}(\sigma, \alpha)=\left(\begin{array}{cc}
I_{\sigma \sigma} & I_{\sigma \alpha} \\
I_{\sigma \alpha} & I_{\alpha \alpha}
\end{array}\right)
$$

com elementos dados por

$$
\begin{aligned}
& I_{\sigma \sigma}=-\frac{1}{\sigma^{2}}+\frac{3 \alpha \kappa_{2}(\alpha)}{\sigma^{2}}-\frac{2(\alpha-1)}{\sigma^{3}}\left[2 a_{11}-\frac{a_{31}}{\sigma^{2}}-\frac{2 a_{22}}{\sigma}\right], \\
& I_{\sigma \alpha}=\frac{1}{\alpha^{2}} \text { e } I_{\alpha \alpha}=\frac{2}{\sigma^{2}} a_{11} .
\end{aligned}
$$

em que $a_{i j}=E\left[T^{i}\left(\frac{\phi(T / \sigma)}{2 \Phi(T / \sigma)-1}\right)^{j}\right]$ pode ser calculado numericamente.

\subsection{Estudo de simulação}

Um simples algoritmo pode ser formulado para gerar valores a partir da distribuição HNP.

(i) Simular $Y \sim U(0,1)$ e

(ii) Calcular $T=\sigma \Phi^{-1}\left(\frac{1+Y^{1 / \alpha}}{2}\right)$.

Tabela 6.1: Médias empíricas e desvios padrão para diferentes valores de $\alpha$.

\begin{tabular}{ccccccc}
\hline \multicolumn{2}{c}{$n=20$} & \multicolumn{2}{c}{$n=50$} & \multicolumn{2}{c}{$n=100$} \\
\hline$\alpha$ & $\hat{\alpha}(D P)$ & $\sqrt{E Q M}$ & $\hat{\alpha}(D P)$ & $\sqrt{E Q M}$ & $\hat{\alpha}(D P)$ & $\sqrt{E Q M}$ \\
\hline 0.8 & $0.834(0.188)$ & 0.139 & $0.814(0.116)$ & 0.093 & $0.804(0.085)$ & 0.068 \\
1.0 & $1.054(0.237)$ & 0.186 & $1.015(0.147)$ & 0.116 & $1.002(0.103)$ & 0.082 \\
2.0 & $2.115(0.506)$ & 0.392 & $2.042(0.312)$ & 0.245 & $2.025(0.203)$ & 0.162 \\
3.0 & $3.189(0.744)$ & 0.582 & $3.084(0.447)$ & 0.353 & $3.047(0.300)$ & 0.240 \\
5.0 & $5.285(1.234)$ & 0.954 & $5.123(0.779)$ & 0.610 & $5.036(0.505)$ & 0.405 \\
\hline
\end{tabular}


A Tabela 6.1 mostra os resultados de estudos de simulações, que ilustram o comportamento do EMVs para 1000 amostras geradas de tamanhos $n=20,50$ e 100 da população com distribuição $H N P(1, \alpha)$. Para cada amostra gerada, os EMVs foram calculados numericamente utilizando o procedimento de Newton-Raphson. Média, desvio padrão (DP) e raiz do erro quadrático méio $(\sqrt{E Q M})$ são apresentadas. Observe que, a medida que o tamanho da amostra aumenta, as estimativas estão mais próximos do verdadeiro valor e, os desvios-padrões e $\sqrt{E Q M}$ se tornam menores, sugerindo a consistência dos estimadores.

\subsection{Ilustração}

Consideramos agora o cálculo de estimadores de máxima verosimilhança para o modelos HNG proposto por Cooray e Ananda [2008] e o modelo HNP baseado em um conjunto de dados real.

\subsubsection{Dados de vulcões}

Os dados reais correspondem a alturas (in 100× pés) de 219 vulcões analizados em Tukey [1977]. Este conjunto de dados foi recentemente analisado em Castillo et al. [2011]. O conjunto de dados completos pode ser encontrado no Apêndice A.1. A Tabela 6.2 apresenta as estatísticas descritivas básicas para os conjuntos de dados.

Tabela 6.2: As estatísticas descritivas para o conjunto de dados das alturas do vulcões

\begin{tabular}{cccccccc}
\hline Dados & Média & Mediana & Moda & DP & Variância & Assimetria & Curtose \\
\hline \hline Vulcões & 70.25 & 65 & 56 & 43.01 & 1850.563 & 0.84 & 3.48 \\
\hline
\end{tabular}

Usando os resultados da Seção 6.3.1, os estimadores de momentos são usados como estimativas iniciais para o abordagem de máxima verosimilhaça.

Tabela 6.3: Estimativas dos parâmetros dos modelos HNG e PHN para o conjuntos de dados dos Vulcões e suas correspondentes estatísticas AIC, CAIC e BIC.

\begin{tabular}{cc|cc|ccc}
\hline Dados & Modelo & $\sigma$ & $\alpha$ & AIC & CAIC & BIC \\
\hline \hline Vulcões & HNP & 70.084 & 1.55 & 2229.835 & 2229.891 & 2236.613 \\
& HNG & 88.736 & 1.297 & 2233.454 & 2233.509 & 2240.232 \\
\hline
\end{tabular}

Tabela 6.4: Testes de bondade de ajuste.

\begin{tabular}{cccc}
\hline Dados & Modelo & \multicolumn{2}{c}{ Estatística } \\
& & $\mathrm{W}^{*}$ & $\mathrm{~A}^{*}$ \\
\hline \hline Vulcões & HNP & 0.017 & 0.172 \\
& HNG & 0.059 & 0.469 \\
\hline
\end{tabular}

A Tabela 6.3 mostra os parâmetros estimados por máxima verosimilhança usando o bbmle pacote no programa R (2014)(R Core Team [2015]). Para cada modelo apresentamos o valor da estimativa de máxima verosimilhança e o correspondente critério de informação de Akaike (AIC), também consideramos o critério de AIC modificado(CAIC) e o critério de informação Bayesiano. Pode-se notar que a AIC, CAIC e BIC mostram melhor ajuste do modelo HNP.

Os modelos ajustados em Castillo et al. [2011] tem aproximadamente AIC 2236.604. A Figura 6.4 apresenta o ajuste para ambos modelos com o histograma e a função de sobrevivência empírica com as estimações dos parâmetros dos modelos HNP e HNG, também mostra a boa concordância com o modelo HNP.

Finalmente, nós aplicamos as estatísticas de teste Cramér-von Mises $\left(W^{*}\right)$ e Anderson-Darling $\left(A^{*}\right)$. 

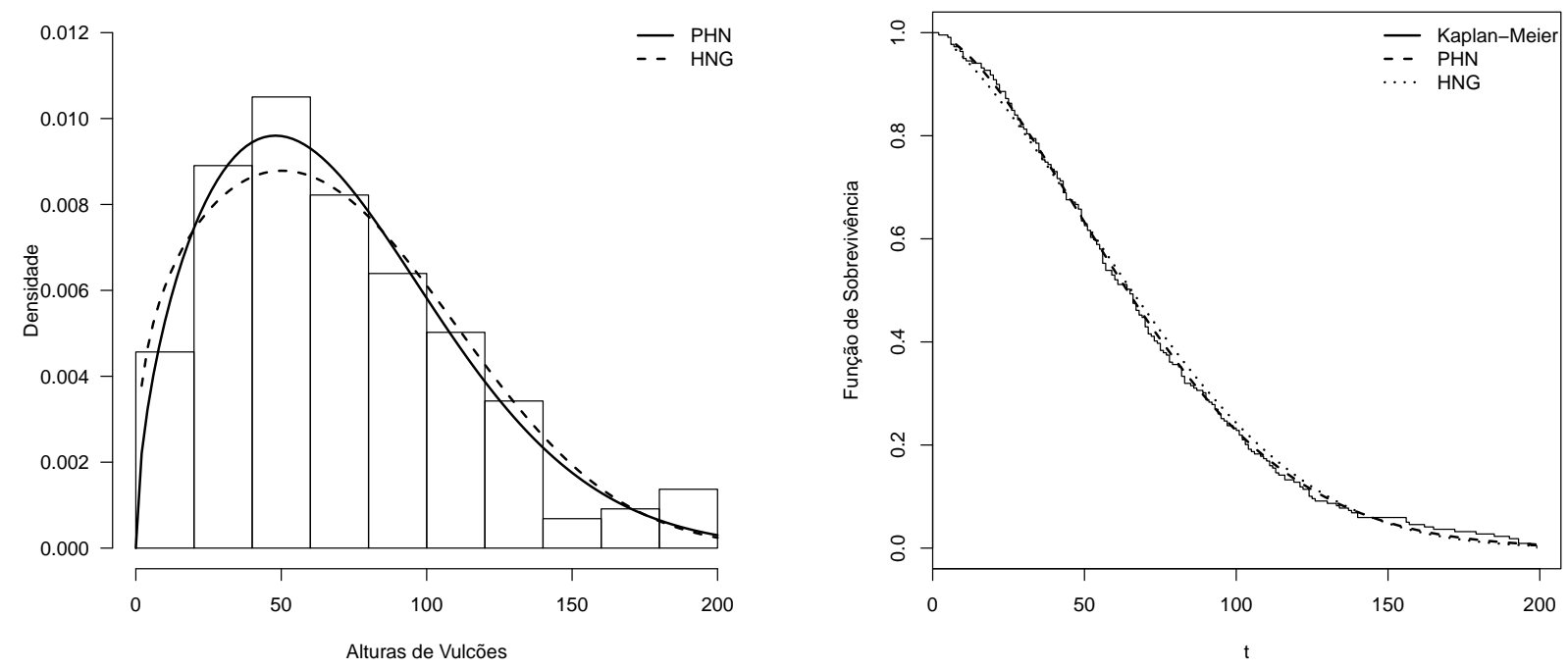

Figura 6.4: Histograma para conjuntos de dados de alturas do vulcão, linhas representam distribuições ajustadas utilizando estimadores de máxima verossimilhança(esquerda) e funções de sobrevivência e a sobrevivência empírica(direita).

Como visto en los capítulos anteriores, quanto menor os valores das estatísticas $W^{*}$ e $A^{*}$, melhor o ajuste para os dados. Os valores destas estatísticas para os modelos são dadas na Tabela 6.4. Como esperado, os valores de $W^{*}$ e $A^{*}$ sugerem que o modelo HNP fornece um melhor ajuste que o modelo HNG para os dados analisados.

\subsubsection{Dados de Engenharia}

O conjunto de dados usados nesta aplicação foi previamente analisado em Birnbaum e Saunders [1958], relacionados com o tempo de vida em ciclos de $10^{-3}$ de alumínio $6061-T 6$ de peças cortadas em ângulo paralelo com o sentido de rotação, oscilando em a taxa de 18 ciclos/s em um máximo de pressão de 31,000psi, com um tamanho de amostra total de 101 unidades. O conjunto de dados completo é dado no Apêndice A.1. A Tabela 6.5 apresenta as estatísticas descritivas básicas para os conjuntos de dados.

Tabela 6.5: Estatísticas descritivas para os dados de Engenharia.

\begin{tabular}{cccccccc}
\hline Dados & Média & Mediana & Moda & DP & Variância & Assimetria & Curtose \\
\hline \hline Engenharia & 133.7 & 133 & 142 & 22.36 & 499.78 & 0.326 & 0.973 \\
\hline
\end{tabular}

Usando os resultados da seção 6.3.1, os estimadores de momentos são usados como estimativas iniciais para o abordagem de máxima verosimilhaça.

Tabela 6.6: Estimativas dos parâmetros do modelo para o conjunto de dado de engenharia e suas correspondentes estatísticas AIC, CAIC e BIC.

\begin{tabular}{cc|cc|ccc}
\hline Dados & Modelo & $\sigma$ & $\alpha$ & AIC & CAIC & BIC \\
\hline \hline Engenharia & HNP & 55.091 & 40.639 & 919.736 & 919.859 & 924.966 \\
& HNG & 148.06 & 4.255 & 943.803 & 943.925 & 949.033 \\
\hline
\end{tabular}

A Tabela 6.6 mostra os parâmetros estimados por máxima verosimilhança usando o bbmle pacote no programa R (2014)(R Core Team [2015]). Pode-se notar que os critérios AIC, CAIC e 

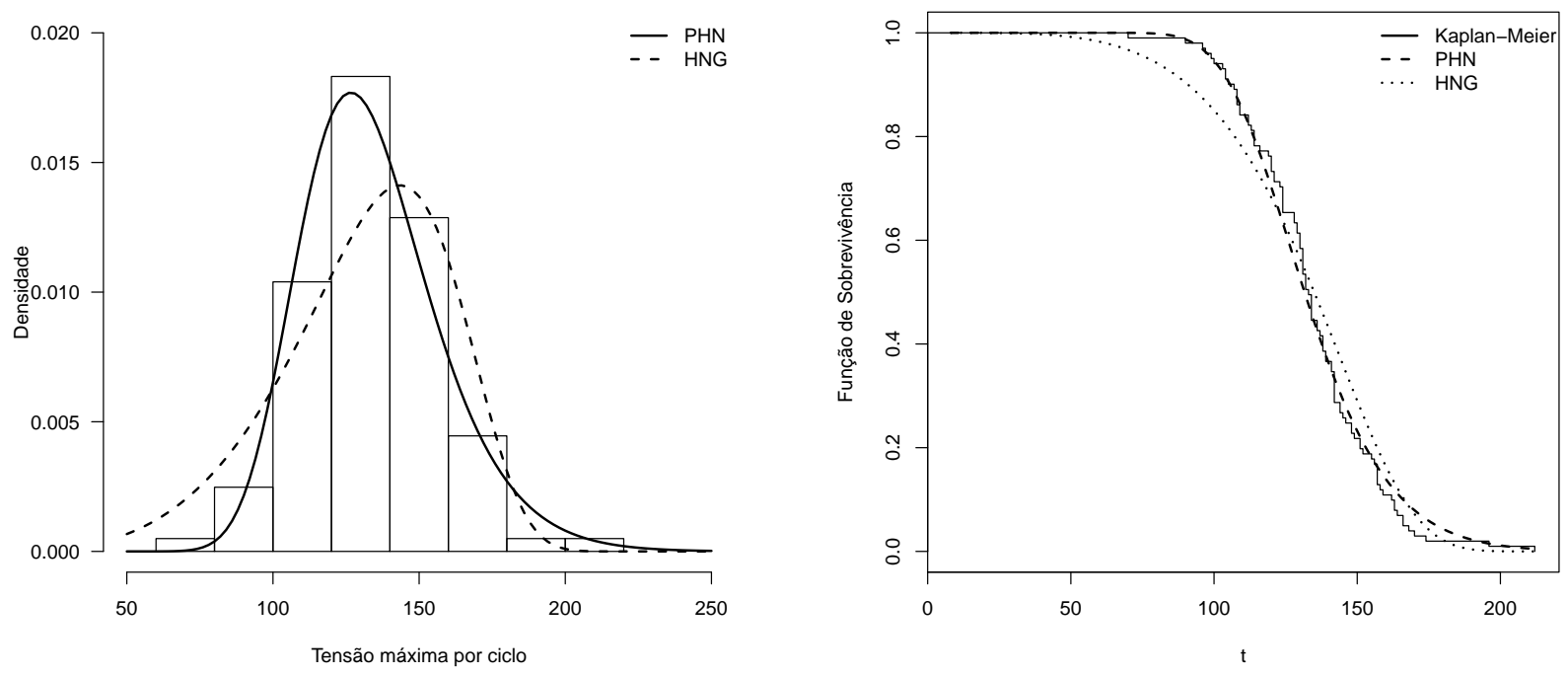

Figura 6.5: Histograma para conjuntos de dados de engenharia, linhas representam distribuições ajustadas utilizando estimadores de máxima verossimilhança(esquerda) e funções de sobrevivência e a sobrevivência empirica(direita).

Tabela 6.7: Testes de bondade de ajuste.

\begin{tabular}{cccc}
\hline Dados & Modelo & \multicolumn{2}{c}{ Estatística } \\
& & $\mathrm{W}^{*}$ & $\mathrm{~A}^{*}$ \\
\hline \hline I3 & HNP & 0.106 & 0.602 \\
& HNG & 0.263 & 1.684 \\
\hline
\end{tabular}

BIC mostram também o melhor ajuste para o modelo HNP.

A Figura 6.5 apresenta o ajuste para ambos modelos com o histograma e a função de sobrevivência empírica com as estimações dos parâmetros dos modelos HNP e HNG, também mostra uma boa concordância para o modelo HNP.

Finalmente, aplicamos as estatísticas de teste Cramér-von Mises $\left(W^{*}\right)$ e Anderson-Darling $\left(A^{*}\right)$. Os valores destas estatísticas para os dois modelos são dadas na Tabela 6.7. Como é esperado, os valores de $W^{*}$ e $A^{*}$ também são menores para o modelo HNP do que o modelo HNG para esse conjunto de dados.

\subsection{Observações finais}

Este capítulo está focado no estudo de um sub-modelo da família de modelos introduzidos em Pescim et al. [2010]. Este modelo tem dois parâmetros e é uma alternativa à half-normal generalizada HNG estudada em Cooray e Ananda [2008]. A maioria dos resultados apresentam expressões explicitas. Um estudo de simulação é conduzida para o parâmetro de forma e se mostra que o EMV apresentar viés pequeno para tamanhos pequeno e médio da amostra. Na aplicação, os critérios AIC, CAIC e BIC são utilizados para comparação de modelos, que mostram que o modelo proposto apresenta melhor ajuste nos conjuntos de dados analisados do que o modelo proposto por Cooray e Ananda [2008]. 


\section{Capítulo 7}

\section{Modelo geométrico half-normal potência com fração de cura}

Neste capitulo considera-se o modelo de cura geométrico apresentado em Rodrigues et al. [2009] usando para $S_{0}(\cdot)$, a função de sobrevivência das células carcinogênicas, uma extensão da distribuição half-normal baseada na distribuição do máximo de uma amostra aleatória. É discutida a estimação de máxima verossimilhança do modelo e finalmente, o modelo é ajustado a um banco de dados reais(Melanoma), comparando-o com outras alternativas para $S_{0}(\cdot)$.

\subsection{Motivação}

Considere a distribuição half-normal potência, discutida no capítulo anterior e em Gómez e Bolfarine [2015]. Lembrando que a função de sobrevivência de $T$ é dada por, em que $T \sim H N P(\sigma, \alpha)$

$$
S_{P H N}(t ; \sigma, \alpha)=1-\left(2 \Phi\left(\frac{t}{\sigma}\right)-1\right)^{\alpha},
$$

em que $\sigma>0$ é o parâmetro de escala, $\alpha>0$ o parâmetro de forma, e $t>0$. Em muitos problemas médicos, tais como as doenças cardíacas crónicas e vários tipos diferentes de câncer, um indivíduo com dano cumulativo pode ser causada por várias causas desconhecidas ou factores de risco. Esta degradação leva a um processo de fadiga, cujos tempos de vida de propagação pode ser adequadamente modelado com a distribuição half-normal potência. O objetivo principal deste capítulo é propor uma nova distribuição, a geométrica potência half-normal com fração de cura (GHNPfc), concebida em um cenário de causas latentes com fração de cura, em que não há informação acerca de qual causa foi responsável pela morte do indivíduo ou pelo reaparecimento do tumor. Note que apenas o valor mínimo dos tempos é observado com uma parte dos indivíduos sendo suscetíveis. A dificuldade surge quando parte da população não é suscetível ao evento de interesse. Por exemplo, em estudos clínicos uma população podem responder favoravelmente a um tratamento, sendo considerados curados. A proporção desta fração da população que não é susceptível ao evento de interesse é geralmente denominado como a fração de cura. Distribuições que acomodam uma fração de curados tem sido amplamente desenvolvidas. Talvez o mais popular tipo de distribuições taxa de cura é a distribuição da mistura introduzida por Boag [1949] e Berkson e Gage [1952]. Esta distribuição, pressupõe que uma certa proporção dos doentes, digamos $p$, são curados, no sentido de que eles não apresentão o evento de interesse e pode ser visto como imune ou curado para a causa da morte em estudo (PerdonA $\tilde{A}_{i}$ e Louzada-Neto [2011]). A referência fundamental sobre as distribuições mistura é Maller e Zhou [1996]. Logo, temos uma distribuição mistura HNP (MHNP) com função de sobrevivência dada por

$$
S_{M H N P}(t)=p_{0}+\left(1-p_{0}\right) S_{H N P}(t), \quad t>0
$$

em que $S_{H N P}(t)$ é dado na equação (7.1) e $p_{0}$ é a fração de cura.

\subsection{Formulação do Modelo}

A distribuição GHNPfc é derivada como segue. Para um indivíduo na população, seja $M$ o número observável de causas (fatores de risco) do evento de interesse para ese indivíduo. Suponha 
que $M$ segue uma distribuição geométrica com parâmetro $\theta$ e função de probabilidade

$$
P(M=m)=(1-\theta) \theta^{m}, \quad m=0,1, \ldots
$$

O tempo da $j$-ésima causa para produzir o evento de interesse é indicada por $Z_{j}, j=1, \ldots, M$. Assumimos que, condicional em $M, Z_{j}$ são independentes e identicamente distribuídas (i.i.d.) com HNP dado na equação (6.1). Além disso, vamos supor que $Z_{1}, Z_{2}, \ldots$ são independentes de $M$. O tempo observável do evento de interesse é definido pelas variáveis aleatórias $T=\min \left(Z_{1}, \ldots, Z_{n}\right)$, e $T=\infty$ se $M=0 \operatorname{com} P(T=\infty \mid M=0)=1$.

Proposição 7.2.1 Sob essa configuração, a função de sobrevivência para a população é dada por

$$
S_{G H N P f c}(t)=\frac{1-\theta}{1-\theta\left[1-\left(2 \Phi\left(\frac{t}{\sigma}\right)-1\right)^{\alpha}\right]}
$$

\section{Prova}

O resultado é inmediato, ao se considerar na função de sobrevivência dada em Rodrigues et al. [2009], a função de sobrevivência dada em (7.1).

A fração de cura é dada por $S_{G H N P f c}(\theta)=1-\theta$ (não é uma função de sobrevivência própria). A função densidade correspondente é dada por

$$
f_{G H N P f_{c}}(t)=\theta(1-\theta) f_{H N P}(t)\left\{1-\theta\left[1-\left(2 \Phi\left(\frac{t}{\sigma}\right)-1\right)^{\alpha}\right]\right\}^{-2}
$$

em que $f_{H N P}(t)$ é a fdp da distribuição half-normal potência. A função de risco da população é dada por

$$
h_{G H N P f c}(t)=\frac{\theta f_{H N P}(t)}{1-\theta\left[1-\left(2 \Phi\left(\frac{t}{\sigma}\right)-1\right)^{\alpha}\right]}
$$

em que $f_{G H N P}(t)$ e $h_{G H N P}(t)$ são funções improprias, pois $S_{G H N P}(t)$ não é uma função de sobrevivência própria. A distribuição em (7.4), pode ser escrita como a distribuição mistura padrão (Berkson e Gage [1952]).

$$
S_{G H N P f c}(t)=(1-\theta)+\theta\left\{\frac{(1-\theta)\left[1-\left(2 \Phi\left(\frac{t}{\sigma}\right)-1\right)^{\alpha}\right]}{1-\theta\left[1-\left(2 \Phi\left(\frac{t}{\sigma}\right)-1\right)^{\alpha}\right]}\right\}
$$

Em seguida, a partir de (7.7) e (7.2), a função de sobrevivência para a populacão não-curada, a partir, do modelo geometrico half-normal potência , é dado por

$$
S_{G H N P}(t)=P(T>t \mid M \geq 1)=\frac{(1-\theta)\left[1-\left(2 \Phi\left(\frac{t}{\sigma}\right)-1\right)^{\alpha}\right]}{1-\theta\left[1-\left(2 \Phi\left(\frac{t}{\sigma}\right)-1\right)^{\alpha}\right]}, \quad t>0
$$

Notamos que $S_{G H N P}(0)=1$ e $S_{G H N P}(\infty)=0$, de modo que (7.8) é a função de sobrevivência prôpria.

A fdp do modelo GHNP é dada por

$$
f_{G H N P}(t)=\frac{(1-\theta) 2 \alpha \phi\left(\frac{t}{\sigma}\right)\left(2 \Phi\left(\frac{t}{\sigma}\right)-1\right)^{\alpha-1}}{\sigma\left(1-\theta\left[1-\left(2 \Phi\left(\frac{t}{\sigma}\right)-1\right)^{\alpha}\right]\right)^{2}}, \quad t>0
$$

A Figura 7.1 apresenta as funções de densidade de probabilidade GHNP para alguns valores fixos de $\theta$. Os gráficos destas figuras mostram que a distribuição GHNP é flexível e que o valor de $\theta$ tem um substancial efeito na sua assimetria e curtose, como se observa na Figura 7.3 .

De (7.9) e (7.8), é fácil verificar que a função de risco do modelo GHNP correspondente para a população não-curada é dada por

$$
h_{G H N P}(t)=\frac{h_{H N P}(t)}{\left(1-\theta\left[1-\left(2 \Phi\left(\frac{t}{\sigma}\right)-1\right)^{\alpha}\right]\right)^{2}}, \quad t>0
$$



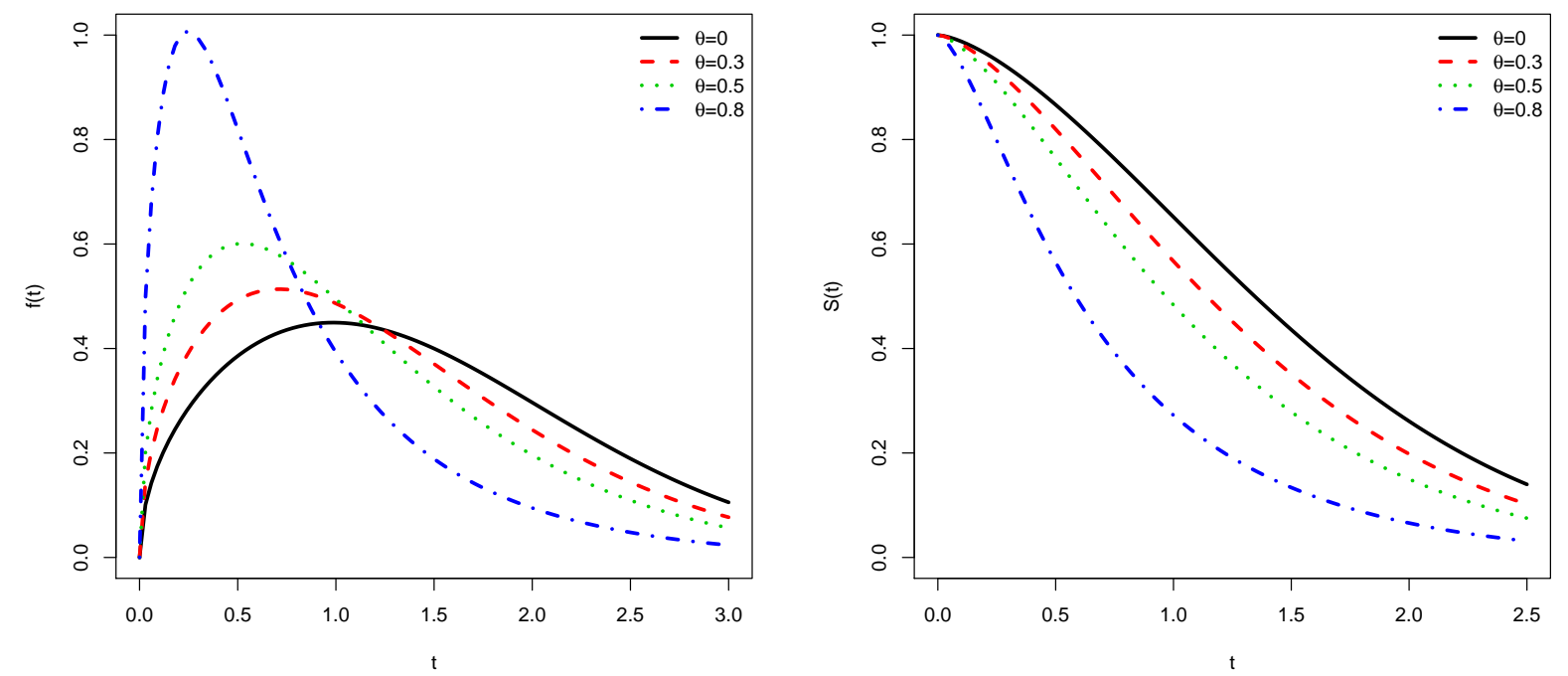

Figura 7.1: Gráficos das funções densidade(esquerda) e sobrevivência(direita) da distribuição geométrica half-normal potência para $\sigma=1.5$, e $\alpha=1.5$.

em que $h_{H N P}(t)$ é a função de risco de uma distribuição HNP.
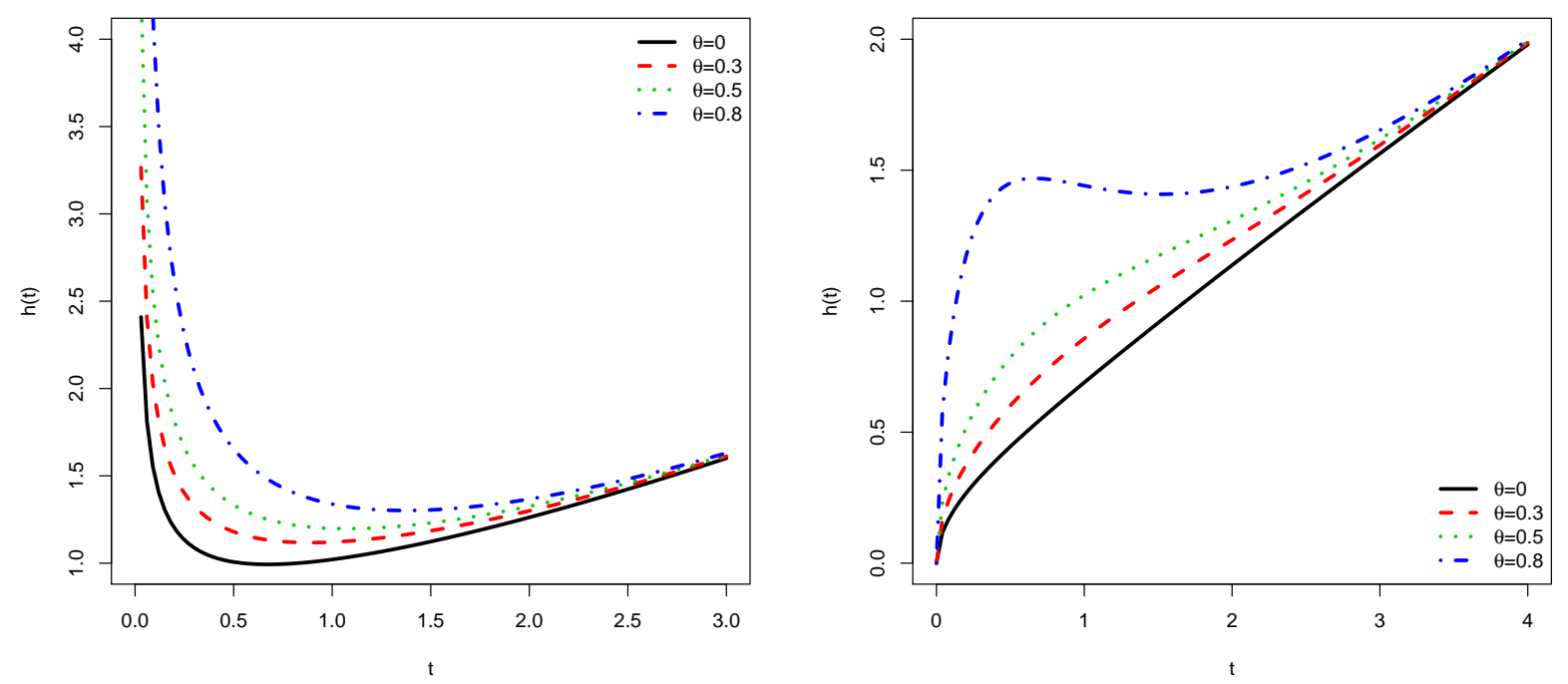

Figura 7.2: Gráficos das funções de risco da distribuição Geométrica Half-Normal Potência para $\sigma=$ $1.5, \alpha=0.5($ esquerda),$\alpha=1.5$ (direita)

\subsubsection{Momentos}

Os momentos do modelo GHNP podem ser calculados numericamente com a rotina de "integrate' do software R. A seguinte proposição apresenta os $r$-ésimos momentos de uma variável aleatória seguindo a distribuição GHNP.

Proposição 7.2.2 Os r-ésimos momentos da variável aleatória $T \sim G P H N(\sigma, \alpha, \theta)$, são dados por

$$
\mu_{r}=E\left(T^{r}\right)=\alpha \sigma^{r}(1-\theta) \eta_{r}(\alpha, \theta), \quad r=1,2, \ldots
$$

em que $\eta_{r}(\alpha, \theta)=\int_{0}^{1} \frac{\left(\Phi^{-1}\left(\frac{1+u}{2}\right)\right)^{r} u^{\alpha-1}}{\left(1-\theta(1-u)^{\alpha}\right)^{2}} d u$, são calculados numericamente. 
Prova A definição do momento implicam

$$
\mu_{r}=E\left(T^{r}\right)=\int_{0}^{\infty} t^{r} \frac{(1-\theta) 2 \alpha \phi\left(\frac{t}{\sigma}\right)\left(2 \Phi\left(\frac{t}{\sigma}\right)-1\right)^{\alpha-1}}{\sigma\left(1-\theta\left[1-\left(2 \Phi\left(\frac{t}{\sigma}\right)-1\right)^{\alpha}\right]\right)^{2}} d t
$$

O resultado segue depois de fazer a mudança de variável $u=2 \Phi\left(\frac{t}{\sigma}\right)-1$.

Corolário 7.2.1 Por conseguinte, os quatro primeiros momentos são dadas por

1. $\mu_{1}=E(T)=\alpha(1-\theta) \sigma \eta_{1}(\alpha, \theta)$

2. $\mu_{2}=E\left(T^{2}\right)=\alpha(1-\theta) \sigma^{2} \eta_{2}(\alpha, \theta)$

3. $\mu_{3}=E\left(T^{3}\right)=\alpha(1-\theta) \sigma^{3} \eta_{3}(\alpha, \theta)$

4. $\mu_{4}=E\left(T^{4}\right)=\alpha(1-\theta) \sigma^{4} \eta_{4}(\alpha, \theta)$

Corolário 7.2.2 Os coeficientes de assimetria e curtose são indicados, respectivamente, por

$$
\sqrt{\beta_{1}}=\frac{\eta_{3}(\alpha, \theta)-3 \alpha(1-\theta) \eta_{1}(\alpha, \theta) \eta_{2}(\alpha, \theta)+2 \alpha^{2}(1-\theta)^{2} \eta_{1}^{3}(\alpha, \theta)}{\sqrt{\alpha(1-\theta)}\left(\eta_{2}(\alpha, \theta)-\alpha(1-\theta) \eta_{1}^{2}(\alpha, \theta)\right)^{3 / 2}}
$$

$e$

$$
\beta_{2}=\frac{\eta_{4}(\alpha, \theta)-4 \alpha(1-\theta) \eta_{1}(\alpha, \theta) \eta_{3}(\alpha, \theta)+6 \alpha^{2}(1-\theta)^{2} \eta_{1}(\alpha, \theta)^{2} \eta_{2}(\alpha, \theta)-3 \alpha^{3}(1-\theta)^{3} \eta_{1}^{4}(\alpha, \theta)}{\alpha(1-\theta)\left(\eta_{2}(\alpha, \theta)-\alpha(1-\theta) \eta_{1}^{2}(\alpha, \theta)\right)^{2}} .
$$

Tabela 7.1: Momentos para algumas combinações de parâmetros da distribuição GHNP.

\begin{tabular}{c|ccccccccccccc}
\hline & \multicolumn{9}{|c}{$\theta=0$} \\
& \multicolumn{2}{|c}{$\sigma=1$} & \multicolumn{4}{c}{$\theta=0.5$} \\
& $\alpha=1$ & $\alpha=2$ & $\alpha=1$ & $\alpha=2$ & $\alpha=1$ & $\alpha=2$ & $\alpha=1$ & $\alpha=2$ & $\alpha=1$ & $\alpha=2$ & $\alpha=1$ & $\alpha=2$ \\
\hline$\mu_{1}$ & 0.798 & 1.128 & 1.596 & 2.257 & 0.586 & 0.624 & 1.172 & 1.247 & 0.364 & 0.274 & 0.729 & 0.548 \\
$\mu_{2}$ & 1 & 1.637 & 4 & 6.547 & 0.627 & 0.859 & 2.507 & 3.435 & 0.313 & 0.357 & 1.252 & 1.429 \\
$\mu_{3}$ & 1.596 & 2.821 & 12.766 & 22.568 & 0.916 & 1.444 & 7.327 & 11.559 & 0.413 & 0.588 & 3.304 & 4.706 \\
$\mu_{4}$ & 3 & 5.547 & 48 & 88.744 & 1.636 & 2.808 & 26.169 & 44.924 & 0.701 & 1.133 & 11.211 & 18.124 \\
$\sqrt{\beta_{1}}$ & $\mathbf{0 . 9 9 5}$ & 0.704 & 0.995 & 0.704 & 1.436 & 1.004 & 1.436 & 1.004 & 2.188 & 2.242 & 2.188 & 2.242 \\
$\beta_{2}$ & $\mathbf{3 . 8 6 9}$ & 3.435 & 3.869 & 3.435 & 5.311 & 3.414 & 5.311 & 3.414 & 9.079 & 7.943 & 9.079 & 7.943 \\
\hline
\end{tabular}

Observação 13 A partir da Tabela \%.1, notamos que para $\alpha=\sigma=1$ e $\theta=0$ os coeficientes de assimetria e curtose coincidem com os coeficientes da distribuição $H N, \sqrt{\beta_{1}}=0.995$ e $\beta_{2}=3.869$.

\subsection{Inferência}

Seja $C_{i}$ o tempo de censura. Observamos $Y_{i}=\min \left\{T_{i}, C_{i}\right\}$ e $\delta_{i}=I\left(T_{i} \leq C_{i}\right)$ é tal que $\delta_{i}=1$ se $T_{i}$ é um tempo para evento, e $\delta_{i}=0$ se é censura a direita, $i=1, \ldots, n$. Seja $\gamma$ o vetor de parâmetros da distribuição do tempo do evento $\mathrm{Z}$ em 7.1. De $n$ pares de tempos e indicadores de censura $\left(y_{1}, \delta_{1}\right), \ldots,\left(y_{n}, \delta_{n}\right)$ a correspondente função de verosimilhança sob a censura não informativa é

$$
L(\gamma, \theta) \propto \prod_{i=1}^{n}\left\{f_{G H N P f c}\left(y_{i} ; \gamma, \theta\right)\right\}^{\delta_{i}}\left\{S_{G H N P f c}\left(y_{i} ; \gamma, \theta\right)\right\}^{1-\delta_{i}},
$$

em que $S_{G H N P f c}\left(y_{i} ; \gamma, \theta\right)$ e $f_{G H N P f c}\left(y_{i} ; \gamma, \theta\right)$ são equações dadas em (7.4) e (7.5) respectivamente. 

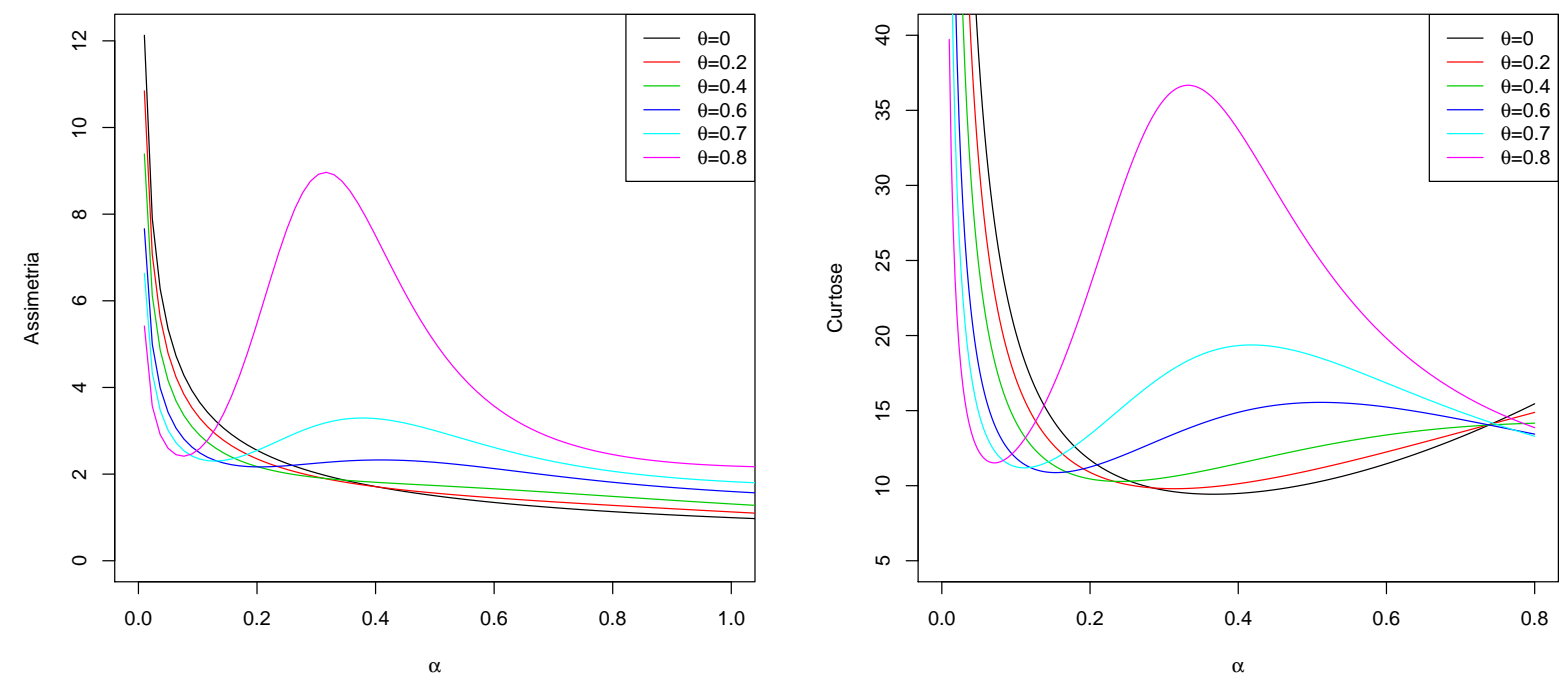

Figura 7.3: Gráficos da assimetria da distribuição geométrica half-normal potência (esquerda) e a curtose (direita)

Nós definimos $1-\theta=p_{0}$. Completando o modelo, associamos a taxa de falhas o vetor de covariadas k-dimensional $\boldsymbol{x}_{i}$ considerando a ligação $\log \left(\frac{p_{0 i}}{1-p_{0 i}}\right)=\boldsymbol{x}_{i}^{T} \boldsymbol{\beta}$, isto é,

$$
p_{0 i}=\frac{\exp \left(\boldsymbol{x}_{i}^{T} \boldsymbol{\beta}\right)}{1+\exp \left(\boldsymbol{x}_{i}^{T} \boldsymbol{\beta}\right)}, \quad, i=1, \ldots, n,
$$

em que o vetor $\boldsymbol{\beta}$ representa o vetor de coeficientes de regressão.

Lembramos que as co-variáveis são tradicionalmente usadas para modelar a esperança do número de causas concorrentes. Por exemplo, no modelo proposto, a partir de (7.3), temos

$$
E\left(N_{i}\right)=\frac{\theta_{i}}{\left(1-\theta_{i}\right)}=\exp \left(\boldsymbol{x}_{i}^{T} \boldsymbol{\beta}\right)
$$

e $p_{0 i}=1-\theta_{i}$, de modo que

$$
p_{0 i}=\frac{1}{\left(1+\exp \left(\boldsymbol{x}_{i}^{T} \boldsymbol{\beta}\right)\right)} .
$$

A ligação entre a fração de curados e as co-variáveis é muito mais complicado do que nesta expressão na ligação logística.

Então, as funções improprias dadas em (7.8) e (7.5) podem ser escritas

$$
S_{G H N P f c}\left(y_{i} ; \gamma, \boldsymbol{\beta}\right)=\left\{1+\left(p_{0 i}^{-1}-1\right) F_{H N P}\left(y_{i} ; \gamma\right)\right\}^{-1}, \quad t>0
$$

e

$$
f_{G H N P f c}\left(y_{i} ; \gamma, \boldsymbol{\beta}\right)=\left\{1+\left(p_{0 i}^{-1}-1\right) F_{H N P}\left(y_{i} ; \gamma\right)\right\}^{-2}\left\{p_{0 i}^{-1}-1\right\} f_{H N P}\left(y_{i} ; \gamma\right), \quad t>0
$$

Baseada na distribuição geométrica com $1-p_{0}$, dada em (7.12)-(7.14), a função de verosimilhança dada em (7.11) pode expressar-se como

$$
L(\theta, \mathfrak{D}) \propto \prod_{i=1}^{n}\left\{\left(p_{0 i}^{-1}-1\right) f_{H N P}\left(y_{i} ; \gamma\right)\right\}^{\delta_{i}}\left\{1+\left(p_{0 i}^{-1}-1\right) F_{H N P}\left(y_{i} ; \gamma\right)\right\}^{-\delta_{i}-1}
$$

em que $\nabla=\left(\beta^{T}, \gamma^{T}\right)^{T}, \mathfrak{D}=(n, y, \delta, X)$, e $X=\left(x_{1}^{T}, \ldots, x_{n}^{T}\right)$

A estimação por máxima verossimilhança do vector de parâmetros $\nabla$ é levada a cabo pela maxi- 
mização numérica directa da função de verossimilhança $l(\nabla ; \mathfrak{D})=\log (L(\nabla ; \mathfrak{D}))$, o que é conseguido usando o software R (veja R Core Team [2015]). Sob condições de regularidade adequadas, pode-se mostrar que a distribuição assintótica do estimador de máxima verossimilhança $\hat{\theta}$ é uma normal multivariada com média o vector $\nabla$ é uma matriz de covariância $\boldsymbol{\Sigma}(\hat{\nabla})$, que pode ser estimada por $\hat{\boldsymbol{\Sigma}}(\hat{\theta})=\left\{-\frac{\partial^{2} l(\theta ; \mathfrak{D})}{\partial \theta \partial \theta^{T}}\right\}^{-1}$, avaliado em $\theta=\hat{\theta}$.

As segundas derivadas são computadas numericamente.

\subsection{Estudo de simulação}

Para avaliar o desempenho do procedimento de estimativa de parâmetros para os modelos propostos, foi realizado um estudo de simulação. Neste estudo considerou-se o modelo proposto com a distribuição HNP para os tempos de eventos (Z) com o parâmetro $\alpha=2$ e $\sigma=1$. Para cada indivíduo $i, 1, \ldots, n$, o número de causas do evento de interesse para essa pessoa $\left(M_{i}\right)$ é gerada a partir da distribuição geométrica com parâmetro,

$$
1-\theta_{i}=p_{0 i}=\exp \left(\beta_{0}+\beta_{1} x_{i}\right) /\left(1+\exp \left(\beta_{0}+\beta_{1} x_{i}\right)\right) .
$$

Em nossas simulações, consideramos uma co-variável binária $x$ com valores extraídos de uma distribuição de Bernoulli com parâmetro 0.5. Tomamos $\beta_{0}=0.5$ e $\beta_{1}=-1$ de modo que a fração de curados para os dois níveis de $x$ são $p_{0}^{(0)}=0.62$ e $p_{0}^{(1)}=0.38$ respectivamente.

Tomamos os tamanhos de amostras $n=50,100,200,400$ e 800. Para cada configuração, realizamos 1000 simulações e calculamos a média das estimativas de máxima verossimilhança (EMVs) da fração de curados $\left(p_{0}^{(0)}\right.$ e $\left.p_{0}^{(1)}\right)$, o desvio padrão $(D P)$ dos EMVs e a raiz quadrada de erros quadrados médios $(\sqrt{E Q M})$ dos EMVs. Os resultados das simulações são apresentados na Tabela 7.2 para os dados simulados a partir do modelo de cura GHNP. Podemos observar que a média dos EMVs estão próximas para os verdadeiros valores do parâmetro, com os DPs e $\sqrt{E Q M}$ diminuindo à medida que o tamanho da amostra aumenta, sugerindo a consistência das estimativas.

Tabela 7.2: Médias das estimativas de máxima verossimilhança (EMVs), desvios padrões (DP) e a raiz quadrada dos erros quadraticos médios $(\sqrt{E Q M})$ da fração de cura $p_{0}^{(0)}$ e $p_{0}^{(1)}$ para dados simulados do modelo de fração de cura HNP.

\begin{tabular}{ccccccc}
\hline$n$ & \multicolumn{2}{c}{ Média dos EMV } & \multicolumn{2}{c}{ DP do EMV } & \multicolumn{2}{c}{$\sqrt{E Q M}$} \\
& $p_{0}^{(0)}$ & $p_{0}^{(1)}$ & $p_{0}^{(0)}$ & $p_{0}^{(1)}$ & $p_{0}^{(0)}$ & $p_{0}^{(1)}$ \\
\hline 50 & 0.6169 & 0.3727 & 0.0974 & 0.0935 & 0.0088 & 0.0095 \\
100 & 0.6230 & 0.3779 & 0.0673 & 0.0664 & 0.0045 & 0.0044 \\
200 & 0.6233 & 0.3765 & 0.0483 & 0.0463 & 0.0024 & 0.0022 \\
400 & 0.6210 & 0.3778 & 0.0334 & 0.0326 & 0.0011 & 0.0011 \\
800 & 0.6223 & 0.3778 & 0.0245 & 0.0234 & 0.0006 & 0.0006 \\
\hline
\end{tabular}

\subsection{Ilustração}

Nesta seção, vamos a ilustrar o nosso modelo a um conjunto de dados reais, empregando a modelagem apresentada na Seção 7.2. O conjunto de dados inclui 205 pacientes observados após a operação para a remoção de melanoma maligno no período de acompanhamento de 15 anos. Estes dados estão disponíveis no pacote timereg no $\mathrm{R}$ (Scheike, 2009). O tempo observado (T) varia de 10 a 5565 dias (de 0.0274 a 15.25 anos, com média $=5.9$ e desvio padrão= 3,1 anos) e refere-se ao tempo até a morte do paciente ou o tempo de censura. Os pacientes mortos por outras causas, bem, como pacientes ainda vivos no final do estudo são observações censuradas (72\%). Consideramos estado de ulceração $\left(x_{1}\right)$ (ausente, $\mathrm{n}=115$; presente, $\mathrm{n}=90$ ) e espessura do tumor $\left(x_{2}\right)$ (em mm. média $=2.92$ e desvio padrão=2.96) como covariáveis. A estimativa de Kaplan-Meier da função de sobrevivência é dada na Figura 7.4. A presença de patamar acima de 0.6 indica que os modelos que ignoram a 
possibilidade de cura não serão adequados para estes dados.

Então, temos a distribuição GHNPfc com $p_{0 i}$ definido em (7.12), assim como a distribuição MHNP com $p_{0 i}$. As estimativas de máxima verossimilhança (EMVs) dos coeficientes dos modelos são dadas na Tabela 7.3. Dita Tabela também apresenta os critérios de seleção nos dois modelos candidatos. De acordo com ambos os critérios, o modelo GPHNfc destaca-se como o melhor e, em seguida, é escolhido para ser o nosso modelo de trabalho.

A gráfico QQ dos resíduos quantílicas normalizados aleatórizados, na Figura 7.5 sugere que o modelo GHNPfc produz um ajuste aceitável.

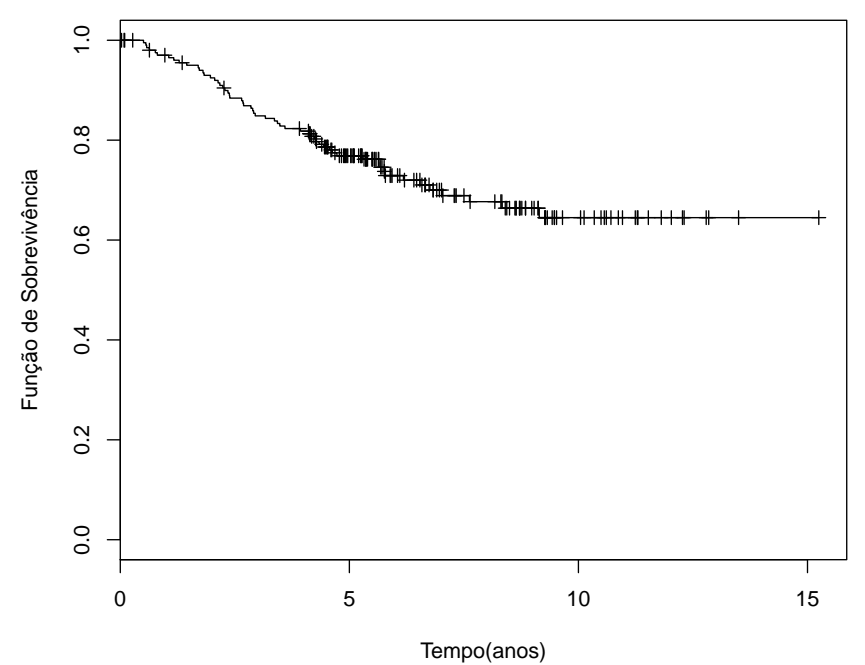

Figura 7.4: Estimador de Kaplan-Meier.

Tabela 7.3: Estimação por Máxima Verossimilhança para os paramêtros estimados para os modelos MPHN e GPHNfc.

\begin{tabular}{cccccc|cc}
\hline \hline Modelo & \multicolumn{5}{c|}{ Estimação } & \multicolumn{2}{c}{ Critêrio } \\
\hline & $\alpha$ & $\sigma$ & $\beta_{\text {intercepto }}$ & $\beta_{\text {espessor }}$ & $\beta_{\text {ulceração }}$ & AIC & BIC \\
\hline GHNPfc & 1.9049 & 5.0041 & 1.8095 & -0.1785 & -1.4804 & 420.86 & 437.472 \\
& $(0.2925)$ & $(1.0788)$ & $(0.3455)$ & $(0.0537)$ & $(0.3569)$ & - & - \\
MHNP & 1.5282 & 4.1679 & 1.2132 & 0.1721 & 1.5335 & 431.39 & 447.995 \\
& $(0.1716)$ & $(0.1551)$ & $(0.3356)$ & $(0.0758)$ & $(0.4112)$ & - & - \\
\hline GBSfc & $\alpha$ & $\sigma$ & $\beta_{\text {intercepto }}$ & $\beta_{\text {espessor }}$ & $\beta_{\text {ulceração }}$ & AIC & BIC \\
& 1.357 & 10.091 & 1.257 & -0.168 & -1.422 & 426.7 & 443.3 \\
MBS & $0.560)$ & $(3.437)$ & $(0.3455)$ & $(0.053)$ & $(0.353)$ & - & - \\
& 1.312 & 6.442 & 2.421 & -0.956 & -1.331 & 430.9 & 447.5 \\
\hline \hline
\end{tabular}

\subsection{Observações finais}

Neste capítulo se propõem-se um novo modelo para os dados de tempo de vida, a distribuição GHNPfc, que é induzida a partir de uma cenário de causas latentes e competitivas com fração de cura.

A importância prática da nova distribuição foi demonstrada em um conjunto de dados reais, em que, usando os critêrios de Akaike e BIC mostramos que o modelo se ajusta melhor aos dados 

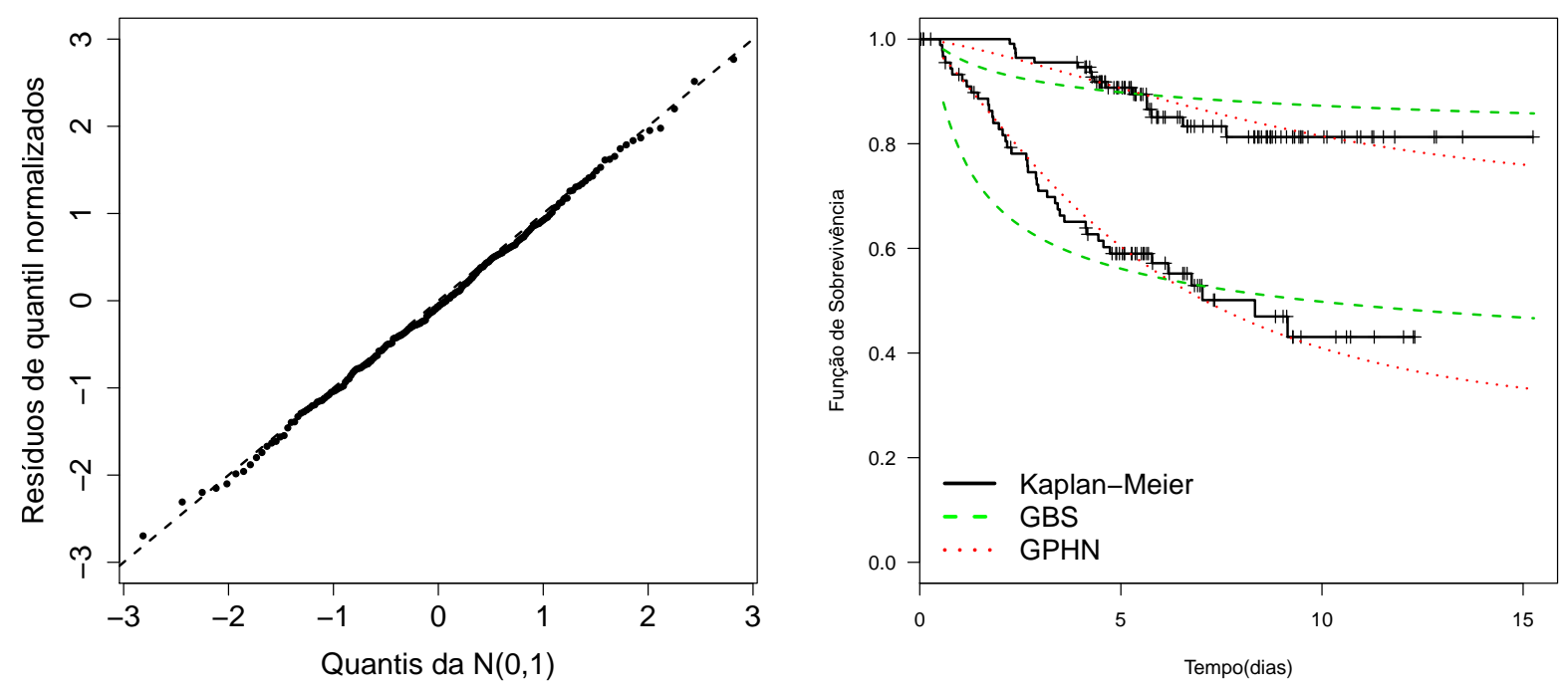

Figura 7.5: Gráficos dos resíduos do modelo (esquerda), e do ajuste do estimador Kaplan-Meier para os pacientes com ulceração e considerando a media do espessor (direita)

de Melanoma é GPHNfc que o modelo MPHN, e que os modelos GBSfc é MBS, propostos por Cancho et al. [2013]. 


\section{Capítulo 8}

\section{Conclusões}

\subsection{Considerações finais}

Nesta tese estudamos algumas distribuções com suporte positivo conhecidas e outras extensões bem conhecidas. No capítulo 2 apresentamos alguns elementos necessários para o desenvolvimento desta tese.

No capítulo 3 consideramos uma extensão da distribuição exponencial com base em misturas de distribuições positivas. Estudamos as principais propriedades desta nova distribuição, com ênfase especial em seus momentos, função geradora de momentos e algumas características relacionadas aos estudos de confiabilidade e análise de sobrevivência. Discutimos também a estimação de parâmetros considerando as abordagens por máxima verossimilhança e momentos. Para avaliar a eficiência dos métodos de estimação um estudo de simulação será conduzido para diferentes tamanhos de amostras. Finalmente, ajustamos o modelo desenvolvido a um conjunto de dados reais. Essa primeira parte do trabalho já está publicada em Gómez et al. [2014].

No capítulo 4 introduzimos a distribuição Rayleigh-Lindley (RL), que é obtida compondo as distribuições Rayleigh e Lindley discreta, em que o procedimento de composição segue a mesma forma que a utilizada por Adamidis e Loukas [1998]. A distribuição resultante é um modelo que contem dois parâmetros competitivo com outros modelos parsimoniosos como as distribuições gama e Weibull. Estudamos algumas propriedades deste novo modelo como os momentos e a vida média residual. A estimação dos parâmetros foi abordada através dos métodos de máxima verossimilhança via o algoritmo EM e os estimadores de momentos. O comportamento desses estimadores foi estudado através de um estudo de simulação. Finalmente, são descritas duas ilustrações a dados reais, a fim de mostrar o desempenho do modelo versus outros dois modelos de dois parâmetros.

No capítulo 5 é proposta uma extensão do modelo exponencial segmentado com base na distribuição do máximo de uma variável aleatória. Propriedades de sua densidade e função de risco são investigadas. Inferência por máxima verosimilhança é discutida. Os resultados de duas aplicações com dados reais são relatadas, em que o modelo é ajustado usando máxima verossimilhança. As aplicações ilustram o melhor desempenho da nova distribuição quando comparada com outros modelos, propostos recentemente na literatura.

No capítulo 6 foi proposto uma extensão da distribuição half-normal com base na distribuição do máximo de uma amostra aleatória. Mostra-se que esta distribuição pertence à família de distribuições beta half-normal generalizada. Propriedades de sua densidade são investigadas, estimadores de máxima verossimilhança são discutidos e a matriz de informação de Fisher é derivada para a situação de dados completos. Na presença de censuras, é usada a matriz de informação observada. Uma ilustração a dados reais foi apresentada e comparações com extensões alternativas da distribuição half-normal foram investigadas. Este capítulo está aceito para publicação em Gómez e Bolfarine [2015].

Finalmente no capítulo 7 foi apresentado o modelo proposto acima, no contexto de modelos de longa duração, isto é, adicionado um parametro da cura, considerando a distribuição geométrica para as causas inicias proposto por Rodrigues et al. [2009]. Foi desenvolvido também estudos de simulação e aplicação com dados reais. Este última trabalho está publicado [Gómez e Bolfarine, 2016]. 


\subsection{Sugestões para pesquisas futuras}

- Implementar o algoritmo EM no modelo exponencial estendido discutido no capítulo 3.

- Estender os modelos antes propostos, no contexto de modelos de longa duração, isto é, considerando a distribuição Binomial Negativa, para as causas inicias, proposto como por (Rodrigues et al. 2009). Procuraremos também desenvolver estudos de simulação e análise de dados reais.

- Considerar um contexto de regressão para alguns dos modelos antes propostos, específicamente no modelo exponencial segmentado definido no capítulo 5. 


\title{
Apêndice A
}

\section{Apêndice}

\section{A.1 Conjunto de Dados.}

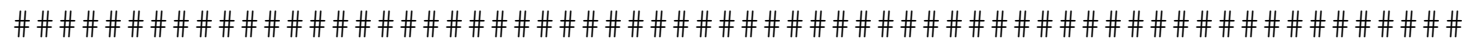

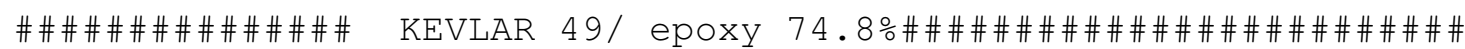

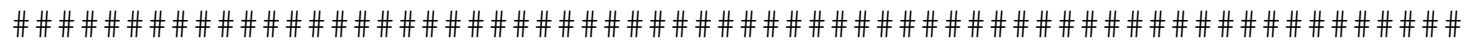
$t<-c(0.0251,0.0886,0.0891,0.2501,0.3113,0.3451$,

$0.4763,0.5650,0.5671,0.6566,0.6748,0.6751,0.6753,0.7696,0.8375$, $0.8391,0.8425,0.8645,0.8851,0.9113,0.9120,0.9836,1.0483,1.0596$, $1.0773,1.1733,1.2570,1.2766,1.2985,1.3211,1.3503,1.3551,1.4595$, $1.4880,1.5728,1.5733,1.7083,1.7263,1.7460,1.7630,1.7746,1.8275$, $1.8375,1.8503,1.8808,1.8878,1.8881,1.9316,1.9558,2.0048,2.0408$, $2.0903,2.1093,2.1330,2.2100,2.2460,2.2878,2.3203,2.3470,2.3513$, $2.4951,2.5260,2.9911,3.0256,3.2678,3.4045,3.4846,3.7433,3.7455$, $3.9143,4.8073,5.4005,5.4435,5.5295,6.5541,9.0960)$
\end{abstract}

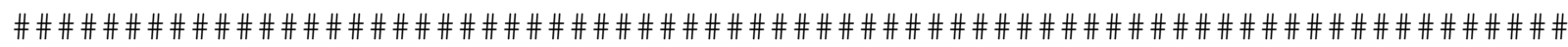
\#\#\#\#\#\#\#\#\#\#\#\#\#\# Tempos de espera em minutos \#\#\#\#\#\#\#\#\#\#\#\#\#\#\#\#\#\#\#\#

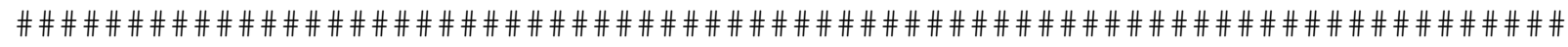
$t<-c(0.8,0.8,1.3,1.5,1.8,1.9,1.9,2.1,2.6,2.7,2.9,3.1,3.2$, $3.3,3.5,3.6,4.0,4.1,4.2,4.2,4.3,4.3,4.4,4.4,4.6,4.7,4.7$, $4.8,4.9,4.9,5.0,5.3,5.5,5.7,5.7,6.1,6.2,6.2,6.2,6.3,6.7$, $6.9,7.1,7.1,7.1,7.1,7.4,7.6,7.7,8.0,8.2,8.6,8.6,8.6,8.8$, $8.8,8.9,8.9,9.5,9.6,9.7,9.8,10.7,10.9,11.0,11.0,11.1,11.2$, $11.2,11.5,11.9,12.4,12.5,12.9,13.0,13.1,13.3,13.6,13.7,13.9$, $14.1,15.4,15.4,17.3,17.3,18.1,18.2,18.4,18.9,19.0,19.9,20.6$, $21.3,21.4,21.9,23.0,27.0,31.6,33.1,38.5)$

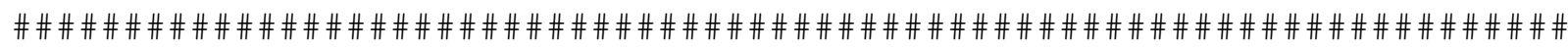

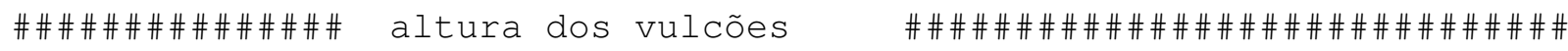

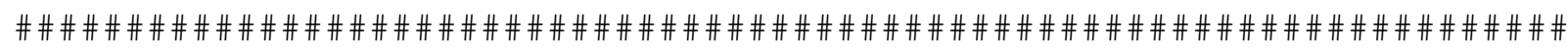
$t<-c(2,5,6,6,6,7,9,9,10,10$,

$10,11,13,16,16,17,19,19,20,20,21,21,22,22,22,24,24$, $24,25,25,26,26,26,27,27,28,28,29,29,29,30,31,31,32$, $32,34,34,35,35,35,35,36,36,36,37,38,39,39,40,41,41$, $41,42,43,43,43,43,43,44,44,44,46,47,48,48,49,49,49$, $49,49,50,50,51,51,52,52,52,53,54,54,55,55,56,56,56$, $56,56,56,57,57,57,59,59,60,60,61,61,64,64,65,65,66$, $66,66,66,67,67,67,68,68,69,70,70,70,70,71,71,71,72$, $73,73,74,75,75,75,76,77,78,78,78,79,81,82,82,82,82$, $83,83,83,85,86,87,89,90,90,90,91,92,93,93,94,94,95$, $95,96,97,97,99,100,101,101,102,102,103,103,104,104$, $105,106,108,109,110,111,111,112,113,113,114,116,116$, 
$119,121,121,122,124,124,124,125,126,130,133,134,137$, $138,140,140,156,156,157,162,165,172,179,185,190,193$, $193,197,199)$

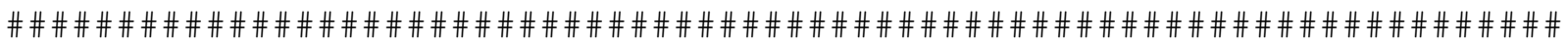
\#\#\#\#\#\#\#\#\#\#\#\#\# Tensão máxima por ciclo de 31.000 psi \#\#\#\#\#\#\#\#\#\#\#\#\#\#\#

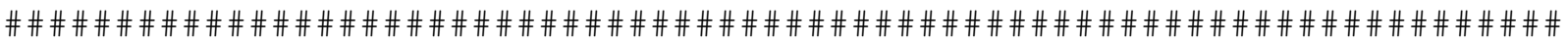

$t<-c(70,90,96,97,99,100,103,104,104,105,107,108,108,108$, 109, 112, 112, 113, 114, 114, 114, 116, 119, 120, 120, 120, 121, 121, 123, 124, 124, 124, 124, 124, 128, 128, 129, 129, 130, 130, 130, 131, $131,131,131,131,132,132,132,133,134,134,134,134,134,136$, $136,137,138,138,138,139,139,141,141,142,142,142,142,142$, $142,144,144,145,146,148,148,149,151,151,152,155,156,157$, $157,157,157,158,159,162,163,163,164,166,166,168,170,174$, $196,212,109)$

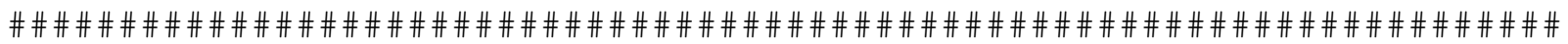
\#\#\#\#\#\#\#\#\#\#\#\#\#\#Tempos de sobrevivência de cobaias\#\#\#\#\#\#\#\#\#\#\#\#\#\#\#\#\#\#\#\#

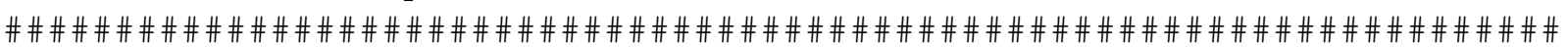
$t<-c(12,15,22,24,24,32,32,33,34,38,38,43,44,48,52,53$, $54,54,55,56,57,58,58,59,60,60,60,60,61,62,63,65,65,67$, $68,70,70,72,73,75,76,76,81,83,84,85,87,91,95,96,98,99$, $109,110,121,127,129,131,143,146,146,175,175,211,233,258$, $258,263,297,341,341,376)$

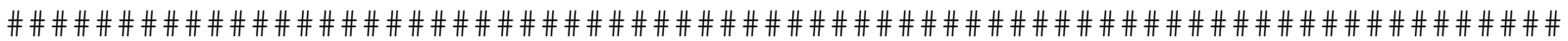

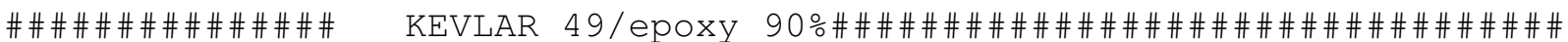

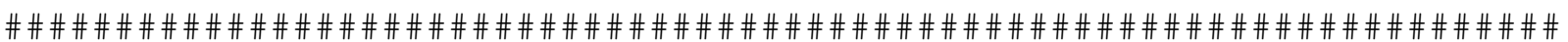
$t<-c(0.01,0.01,0.02,0.02,0.02,0.03,0.03,0.04,0.05,0.06,0.07$, $0.07,0.08,0.09,0.09,0.10,0.10,0.11,0.11,0.12,0.13,0.18,0.19$, $0.20,0.23,0.24,0.24,0.29,0.34,0.35,0.36,0.38,0.40,0.42,0.43$, $0.52,0.54,0.56,0.60,0.60,0.63,0.65,0.67,0.68,0.72,0.72,0.72$, $0.73,0.79,0.79,0.80,0.80,0.83,0.85,0.90,0.92,0.95,0.99,1.00$, $1.01,1.02,1.03,1.05,1.10,1.10,1.11,1.15,1.18,1.20,1.29,1.31$, $1.33,1.34,1.40,1.43,1.45,1.50,1.51,1.52,1.53,1.54,1.54,1.55$, $1.58,1.60,1.63,1.64,1.80,1.80,1.81,2.02,2.05,2.14,2.17,2.33$, $3.03,3.03,3.34,4.20,4.69,7.89$.

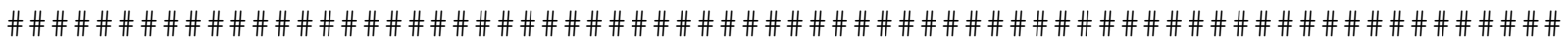

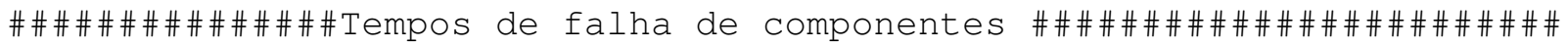

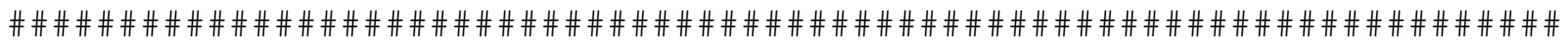
$t<-(0.061,0.073,0.075,0.084,0.086,0.087,0.088,0.089,0.089$, $0.089,0.099,0.102,0.117,0.118,0.119,0.120,0.123,0.135,0.143$, $0.168,0.183,0.185,0.191,0.192,0.199,0.203,0.213,0.215,0.257$, $0.258,0.275,0.297,0.297,0.298,0.299,0.308,0.314,0.315,0.330$, $0.374,0.388,0.403,0.497,0.714,0.790,0.815,0.817,0.859,0.909$, $1.286)$ 


\section{Referências Bibliográficas}

Aarset, M.V.(1987). How to identify bathtub hazard rate, IEEE Transactions on Reliability 36:106108.

Abouammoha, A.M., Alshangitia, Arwa M., Ragabb, I.E. (2015) A new generalized Lindley distribution, Journal of Statistical Computation and Simulation. 85:3662-3678

Adamidis, K., Loukas, S. (1998). A lifetime distribution with decreasing failure rate, Statistics and Probability Letters. 39:35-42.

Ahsanullah, M., Golam Kibria, B.M. and Shakil, M. (2014). Normal and Student's t Distributions and Their Applications, Atlantis Press.

Akaike, H.(1973). Information theory and an extension of the maximum likelihood principle, Second International Symposium on Information Theory, Budapest:Akademinai Kiado. 267-281.

Akaike, H. (1974). A new look at the statistical model identification, IEEE Transactions on Automatic Control. 19:716-723.

Anderson, T.W. and Darling, D.A.(1952). Asymptotic theory of certain "goodness of fit" criteria based on stochastic processes, The Annals of Mathematical Statistics. 23:193-212.

Andrews, D.F., Herzberg, A.M. (1985). Data: A Collection of Problems from Many Fields for the Student and Research Worker, New York: Springer.

Arnold, B.C. (2004). Discussion of Jones, M.C. Families of distribution arising from distributions of order statistics, Test. 13:23-25.

Apostol T.M.(2010). Zeta and related functions, In: F.W.F. Olver, D.W. Lozier, R.F. Boisvert, C.W. Clark (Eds.), NIST Handbook of Mathematical Functions, National Institute of Standards and Technology, Washington, DC, and Cambridge University Press, Cambridge. 601-616.

Azzalini, A. (1985). A class of distributions which includes the normal ones, Scandinavian Journal Statistics. 12:171-8.

Azzalini, A. (1986). Further results on a class of distributions wich includes the normal ones, Statistics. 46:199-208.

Bakouch, H.S., Jazi, M.A., Nadarajah, S. (2014). A new discrete distribution, Statistics. 48:200-240.

Barlow, R.E., Toland, R.H., Freeman, T. (1984). A Bayesian analysis of stress-rupture life of kevlar 49/epoxy spherical pressure vessels, In: Proc. Canad. Conf. Appl. Statist. New York: Marcel Dekker.

Berkson, J., Gage, R. (1952). Survival curve for cancer patients following treatment, Journal of the American Statistical Association. 47:501-515.

Birnbaum, Z. W. and Saunders, S. C. (1958) A statistical model for lifelength of materials. Journal of the American Statistical Association. 53:151-160. 
Bjerkedal, T. (1960) Acquisition of resistance in guinea pigs infected with different doses of virulent tubercle bacilli, American Journal of Hygiene. 72:130-148.

Boag, J.W. (1949). Maximum likelihood estimates of the proportion of patients cured by cancer therapy, Journal of the Royal Statistical Society B. 11:15-53.

Bozdogan, H. (1987). Model selection and Akaike's Information Criterion (AIC): The general theory and its analytical extensions, Psychometrika. 52:345-370.

Cancho, V.G., Louzada, F., Barriga, G.D.C. (2013). The geometric Birnbaum-Saunders regression model with cure rate, Journal of Statistical Planning and Inference. 142:993-1000.

Castillo, N.O., Gómez, H.W., Leiva, V. and Sanhueza, A. (2011). On the Fernández-Steel distribution: Inference and application, Computational Statistics 63 Data Analysis. 55:2951-2961.

Corless, R.M., Gonnet, G.H., Hare,D.E.G., Jeffrey,D.J., Knuth. D.E. (1996) On the Lambert W function. Advances in Computational Mathematics, 5,329-359.

Chen, G., Balakrishnan, N., (1995). A general purpose approximate goodness-of-fit test, Journal of Quality Technology. 27:154-161.

Colosimo, E. A., Giolo, S. R. (2006). Análise de Sobrevivência Aplicada, Edgard Blucher, São Paulo.

Cooray, K., Ananda, M.M.A. (2008). A generalization of the half-normal distribution with applications to lifetime data, Communications in Statistics - Theory 63 Methods. 37:1323-1337.

Cordeiro, G.M., Pescim, R.R., Ortega, E.M.M. (2012). The Kumaraswamy generalized half-normal distribution for skewed positive data, Journal of Data Science 10,195-224.

Cordeiro G.M., Cristino C.T., Hashimoto E.M., Ortega E.M. (2013). The beta generalized Rayleigh distribution with applications to lifetime data, Statistical Papers. 54(1):133-161.

Cordeiro, G. M. and de Castro, M. (2015). A new family of generalized distributions, Journal of Statistical Computation and Simulation 81:883-898.

Demarqui, F. N., Loschi, R. H. and Colosimo, E. A.(2008). Estimating the grid of time-points for the piecewise exponential model, Lifetime Data Analysis. 14:333-356.

Dempster AP, Laird NM, Rubin DB.(1997). Maximum likelihood from incomplete data via the EM algorithm, Journal of the Royal Statistical Society, Series B 39:1-38.

Durrans, S.R. (1992). Distributions of fractional order statistics in hydrology, Water Resources Research. 28:1649-1655.

Eugene, N., Lee, C. and Famoye, F. (2002). Beta-normal distribution and its applications, Communications in Statistics-Theory and Methods. 31:497-512.

Feigl, P. e Zelen, M. (1965). Estimation of exponential probabilities with concomitant, Biometrics. 21:826-838.

Friedman, M. (1982). Piecewise exponential models for survival data with covariates, The Annals of Statistics. 10:101-113.

Feinleib, M.(1960). A method of analyzing log normally distributed survival data with incomplete follow-up, Journal of the American Statistical Association. 55:534-545.

Findley, D.F.(1985). On the unbiasedness property of aic for exact or approximating linear stochastic time series models, Journal of Time Series Analysis. 6:229-252. 
Ghitany ME, Atieh B, Nadarajah S. (2008) Lindley distribution and its application. Math Comput Simul. 78(4),493-506.

Gómez, Y.M., Bolfarine, H. and Gómez, H.W. (2014). A new extension of the exponential distribution, Colombian Journal of Statistics. 37,25-34.

Gómez, Y.M., Bolfarine, H. (2015). Likelihood-based inference for power half-normal distribution, Journal of Statistical Theory and Applications. 14:383-398.

Gómez, Y.M., Bolfarine, H. (2016). The heometric power half-normal regression model with cure rate, Hacettepe Journal of Mathematics and Statistics. DOI:10.15672/HJMS.201613820026.

Gupta, D. and Gupta, R.C. (2008). Analyzing skewed data by power normal model,Test. 17:197210.

Gupta, R.C., Kannan, N., Raychoudhuri, A. (1997). Analysis of log-normal survival data, Mathematical Bioscience. 139:103-115.

Gupta, R. D. and Kundu, D. (1999). Generalized exponential distributions, Australian and New Zealand Journal of Statistics. 41:173-188.

Gupta, R. D. and Kundu, D. (2001). Exponentiated exponential family: An alternative to gama and Weibull distribution, Biometrical Journal. 43(1):117-130.

Hashimoto, E.M., Ortega, E.M.M. and Cordeiro G.M.(2013). Modelo de Regressão Gama-G em Análise de Sobrevivência.

Henze, N. (1986). A probabilistic representation of the skew-normal distribution, Scandinavian Journal of Statistics 13:271-5.

Horner, R. D. (1987). Age at onset of alzheimer's disease: Clue to the relative importance of etiologic factors, American Journal of Epidemiology. 126:409-414.

Hurvich, C.M. and Tsai,C.L. (1989). Regression and time series model selection in small samples, Biometrika. 76:297-307.

Iriarte Y.A., Gómez, H.W., Varela H., Bolfarine H. (2015). Slashed Rayleigh distribution, Colombian Journal of Statistics. 38:31-44.

Jodrá P., Jiménez-Gamero M.D. (2014). On a logarithmic integral and the moments of order statistics from the Weibull-geometric and half-logistic families of distributions, Journal of Mathematical Analysis and Applications. 410:882-890.

Kalbfleisch J.D., Prentice R.L. (2002). The Statistical Analysis of Failure Data, Wiley, New York, second edition.

Klein, J.P., Moeschberger, M.L. (2003). Survival Analysis: Techniques for Censoring and Truncated Data, Springer New York, second edition.

Kolmogorov, A. (1933). Sulla determinazione empirica di uma legge di distribuzione, Giornale dell'Istituto Italiano degli Attuari. 4:1-11.

Kundu D., Raqab M.Z. (2005). Generalized Rayleigh distribution: different methods of estimation, Computational Statistics \& Data Analysis. 49,:187-200.

Kundu, D., Kannan, N., and Balakrishnan, N. (2008). On the hazard function of Birnbaum-Saunders distribution and associated inference, Computational Statistics 85 Data Analysis. 52:2692-2702.

Kus, C. (2007). A new lifetime distribution, Computational Statistics $\&$ Data Analysis. 51:44974509 . 
Lehmann, E.L. (1953). The power of rank tests, Annals of Mathematical Statistics. 24:23-43.

Lee, S.Y., Xu, L. (2004). Influence analyses of nonlinear mixed-effects models, Computational Statistics 83 Data Analysis. 45:321-341.

Lin, T.I., Lee, J.C., Hsieh, W.J. (2007). Robust mixture modeling using the skew t distribution, Statistics and Computing. 17:81-92.

Lindley, D.V.,1958. Fiducial distributions and Bayes' theorem. Journal of the Royal Statical Society, Series A. 20:102-107.

Lagakos, S.W. (1979). A stochastic model for censored-survival data in the presence of an auxiliary variable, Biometrics. 35:139-156.

Louzada-Neto, F., Mazucheli, J., Achcar, J.A. (2001). Uma Introdução à Análise de Sobrevivência e Confiabilidade, XXVII Jornadas Nacionales de Estadística, Chile.

Manesh, S.F., Khaledi, B.E. (2008). On the likelihood ratio order for convolutions of independent generalized Rayleigh random variables, Statistics $\& 3$ Probability Letters. 78:3139-3144.

Maller, R.A., Zhou, S. (1996). Survival Analysis with Long Term Survivors, Wiley, New York.

Miller, R.G. (1974). The jackknife: a review, Biometrika. 61:1-15

Morais, A.L., Barreto-Souza, W. (2014). A compound class of Weibull and Power Series distribution, Computational Statistics and Data Analysis. 55:1410-1425.

Mosteller, F., Tukey, J.W. (1977). Data Analysis and Regression. Addison-Wesley, Reading, MA.

Mudholkar, G.S., Srivastava, D.K. (1993). The exponentiated Weibull family: a reanalysis of the bus-motor-failure data, Technometrics. 37:436-445.

Mudholkar, G.S, Srivastava, D.K., Kollia, G.D. (1996). A generalization of the Weibull distribution with application to the analysis of survival data, Journal of the American Statistical Association. 91:1575-1583.

Murthy, D.P., Xie, M., Jiang, R. (2004). Weibull models, Wiley, New York.

Nadarajah, S. and Kotz, S. (2006). The beta exponential distribution, Reliability Engineering and System Safety. 91:689-697.

Nadarajah, S., Kotz, S. (2006). The exponentiated type distributions, Acta Applicandae Mathematicae. 92:97-111.

Nadarajah, S. and Haghighi, F. (2011). An extension of the exponential distribution, Statistics. 45:543-558.

Nakagawa, T. and Osaki, S.(1975). Discrete Weibull distribution, IEEE Transactions on Reliability. 24:300-301.

Pan, R., Kozakai, Y. (2013). Semiparametric model and Bayesian analysis for clustered accelerate life testing data, Statistical Research Letters. 2:1-11.

Perdona, G.S.C., Louzada-Neto, F. (2011). A general hazard model for lifetime data in the presence of cure rate, Journal of Applied Statistics. 38:1395-1405.

Pescim, R.R., Demétrio, C.G.B., Cordeiro, G.M., Ortega, E.M.M and Urbano, M.R. (2010). The beta generalized half-normal distribution, Computational Statistics and Data Analysis.54:945957. 
Pewsey, A., Gómez, H.W. and Bolfarine, H. (2012). Likelihood-based inference for power distributions, Test. 21:775-789.

R Core Team, 2015. R: A language and environment for statistical computing, R Foundation for Statistical Computing, Vienna, Austria. URL http://www.R-project.org/.

Rodrigues, J., Cancho, V.G., de Castro, M., Louzada-Neto, F. (2009). On the unification of the long-term survival models. Statistics and Probability Letters. 79:753-759.

Rogers, W.H., Tukey, J.W. (1972). Understanding some long-tailed symmetrical distributions, Statistica Neerlandica. 26:211-226.

Rosin, P. and Rammler, B.(1933). The laws governing the fineness of powdered coal, Journal Institute Fuels. 6:29-36.

Schwarz, G. (1978). Estimating the dimension of a model, Annals of Statistics, 6:461-464.

Sen, P.K., Singer, J.M., Pedroso-de-Lima, A.C. (2010). From Finite Sample to Asymptotic Methods in Statistics. Cambridge University Press: New York.

Shawky,AI and Abu-Zinadah,H.H. (2008). Characterizations of the exponentiated pareto distribution based on record values, Applied Mathematical Sciences. 2:1283-1290.

Siddiqui, M. M. (1964). Statistical inference for Rayleigh distributions, The Journal of Research of the National Bureau of Standards, Sec. D: Radio Science. 68D:1007

Smirnov, N. (1939). On the estimation of the discrepancy between empirical curves of distribution for two independent samples, Bulletin Mathématique de l'Université de Moscou. 2:2.

Sugiura, N.(1978). Further analysts of the data by Akaike's information criterion and the finite corrections, Communications in Statistics-Theory and Methods. 7:13-26.

Tahmasbi, R., Rezaei, S., (2008). A two-parameter lifetime distribution with decreasing failure rate, Computational Statistics \&3 Data Analysis. 52:3889-3901.

Tukey, J.W. (1977). Exploratory Data Analysis, Addison-Wesley, Reading, MA.

Yang, Z. (1994). Maximum likelihood phylogenetic estimation from DNA sequences with variable rates over sites: Approximate methods, Journal of Molecular Evolution. 39:306-314. 